Master Thesis

Software Engineering

September 2011

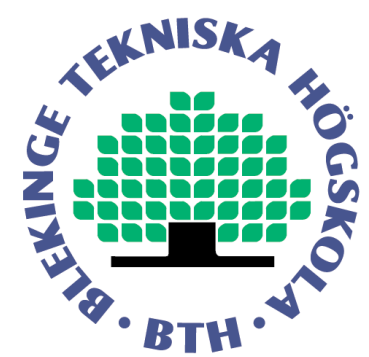

\title{
Obsolete Software Requirements
}

\author{
Showayb A. A. Zahda
}


This thesis is submitted to the School of Engineering at Blekinge Institute of Technology in partial fulfillment of the requirements for the degree of Master of Science in Software Engineering. The thesis is equivalent to 16 weeks of full time studies.

\section{Contact Information:}

Author:

Showayb A A Zahda

E-mail: shuaib.zahda@gmail.com

External advisors:

Krzysztof Wnuk

Lund University

Address: Box 118, 22100 Lund

Phone: +46 462224517

Alberto Sillitti

Free University of Bolzano

Address: Via della Mostra/Mustergasse 4, Bolzano/Bozen , Italy , I-39100

Phone: +390471016134

University advisor:

Tony Gorschek

School of Computing

School of Computing Blekinge Institute of Technology

SE-371 79 Karlskrona

Sweden
Internet : www.bth.se/com

Phone : : +46455385000

Fax : : $\quad+46455385057$ 


\begin{abstract}
Context. Requirements changes are unavoidable in any software project. Requirements change over time as software projects progress, and involved stakeholders (mainly customers) and developers gain better understanding of the final product. Additionally, time and budget constraints prevent implementing all candidate requirements and force project management to select a subset of requirements that are prioritized more important than the others so as to be implemented. As a result, some requirements become cancelled and deleted during the elicitation and specification phase while other requirements are considered not important during the prioritization phase. A common scenario in this situation is to leave the excluded requirements for being considered in the next release. The constant leaving of the excluded requirements for the next release may simply render them obsolete.

Objectives. In this master thesis, a preliminary investigation is conducted to study the phenomenon of obsolete software requirements (OSRs). The major objectives of the thesis are:

1. To find if OSRs exist in reality and how serious they are.

2. To define an OSR.

3. To propose processes to discover OSRs and to take possible actions against them when they are discovered.

4. To examine the effects of OSRs on project's type and size.

5. To insert/inject OSRs into the requirements engineering life-cycle.

6. To develop a classification for requirements that can help finding OSRs.

7. To find empirical evidence regarding OSRs in the industry about all the above mentioned objectives.
\end{abstract}

Methods. A systematic literature review (SLR) is conducted to find relevant information about OSRs. The articles were retrieved from a number of sources including Compendex, Inspec, IEEE Xplore, and ACM Digital Library. Besides, a web-based questionnaire was distributed to experts from both of the industry and the academia in order to collect empirical information about OSRs. The data retrieved from the questionnaire was analyzed mainly using descriptive analysis and chi-square test of significance.

Results. The phenomenon of OSRs exists in reality and is deemed serious by the questionnaire respondents. Moreover, the thesis suggests a definition for OSRs based on the results of the questionnaire. Another contribution is proposing several processes to find OSRs in the requirements' documents, as well as suggesting possible actions to be taken against them. Additionally, OSRs are found to have negative effects on software projects. These effects vary depending on the project's type and size.

Conclusions. In conclusion, the phenomenon of OSRs does exist in reality, and is considered serious by the majority of the questionnaire respondents. The respondents also believe that OSRs have negative effects on software projects depending on the size of the projects (measured in number of requirements) and the type of the project (outsourced, market-driven, and bespoke projects). In addition to, this thesis presents the definition of OSRs based on the experience of the author and the results of the questionnaire. As well, he suggests processes to find/discover OSRs based on several factors such as the volatility value, requirements' types, version control system, and others. In the same manner, he suggests ways of treating OSRs after being discovered such as keeping them, assigning a requirements' status "obsolete", and providing the rationale why a requirement becomes obsolete. Finally, OSRs could be discovered at any stage of the requirements engineering life-cycle mainly during requirements change, requirements analysis, and requirements validation phases.

Keywords: obsolete requirements, volatile requirements, requirements engineering, requirements management. 


\section{ACKnowledgement}

I would like to extend my gratitude to my supervisor Krzysztof Wnuk for his fruitful and invaluable support, feedback and help from the inception of the thesis till its end. Wnuk's help is endless, he did not only supervise my thesis, but he also advised and guided me in other matters of life outside the scope of the academia.

I would like to take the opportunity to thank my cosupervisors Dr. Tony Gorschek and Dr. Sillitti Alberto for their comments and feedback.

This master thesis is part of my European Master in Software Engineering that is sponsored by Erasmus Mundus and the European Commission. Without their financial support, this thesis could not become a reality. Thank you very much.

Recognition should be given to all the participants of the web questionnaire from all over the world. Indeed, without their participation, this master thesis would not have seen the lights.

Last but not least, I would like to thank all my family members who supported me throughout my studies, my friends all over the world and to everyone who contributed to the success of this work directly or indirectly. 


\section{Table of Contents}

1.INTRODUCTION

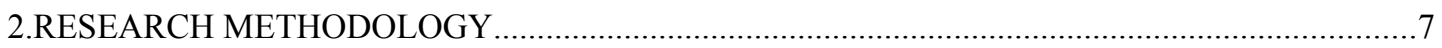

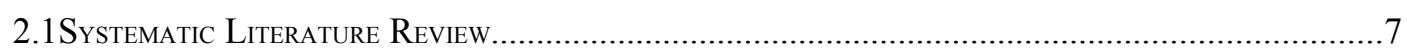

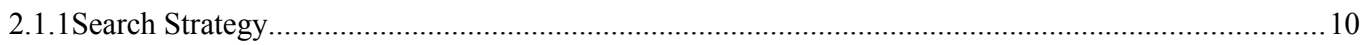

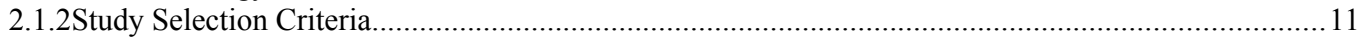

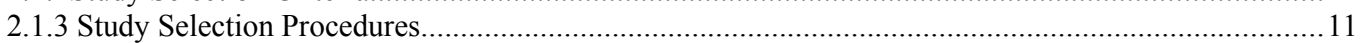

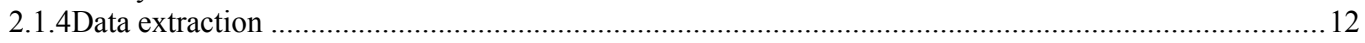

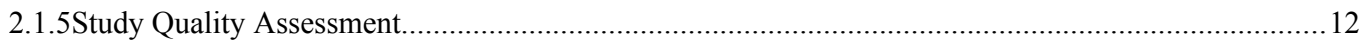

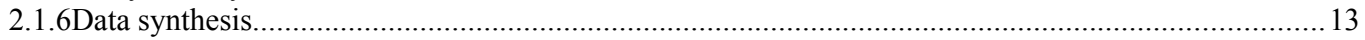

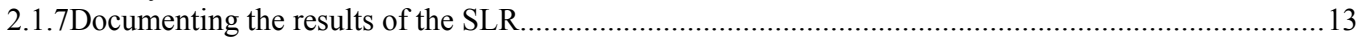

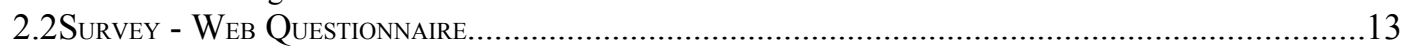

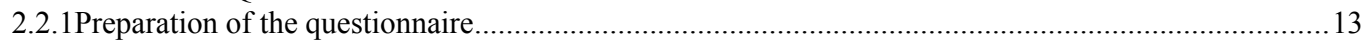

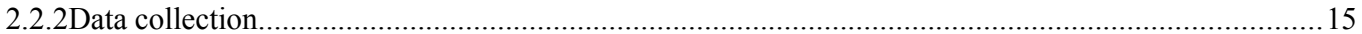

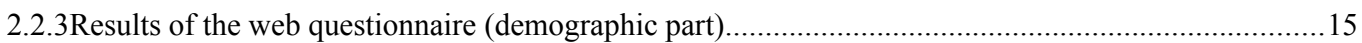

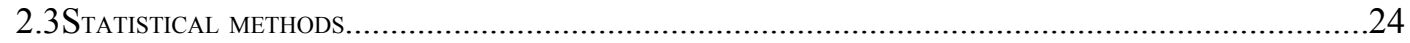

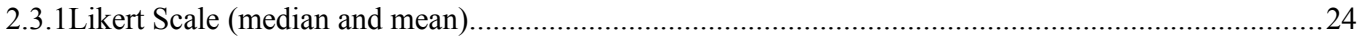

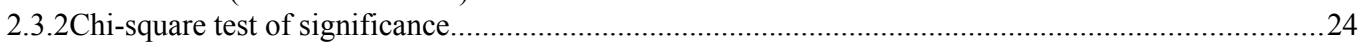

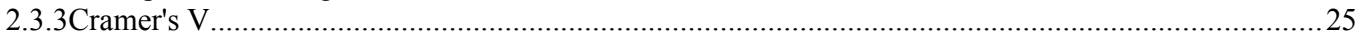

3.OBSOLETE SOFTWARE REQUIREMENTS DEFINITION AND EXISTENCE IN

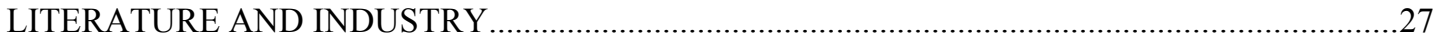

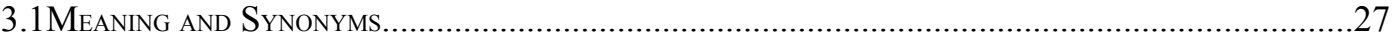

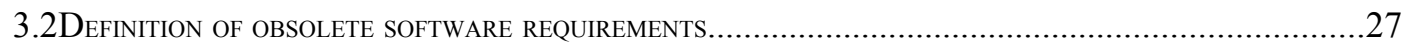

3.3OBSOLETE SOFTWARE REQUIREMENTS IN THE LITERATURE ..........................................................

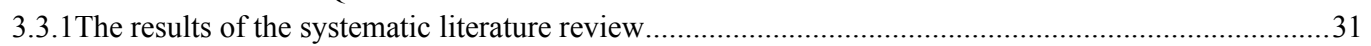

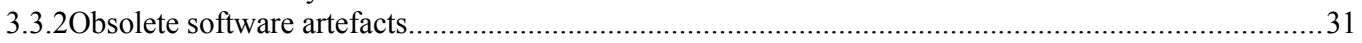

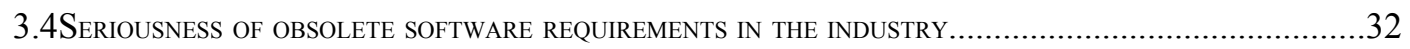

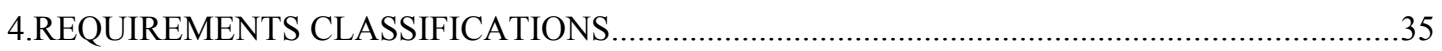

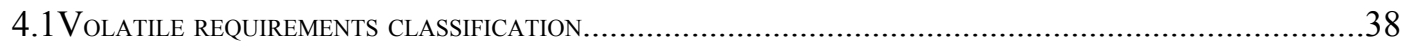

5.OBSOLETE SOFTWARE REQUIREMENTS PROCESSES ......................................................43

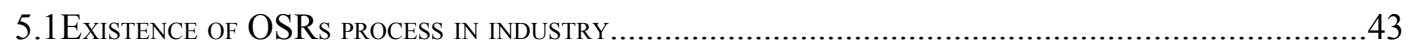

5.2SUGGESTED PROCESSES FOR OBSOLETE SOFTWARE REQUIREMENTS ..............................................48

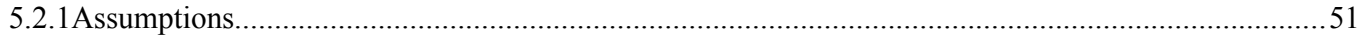

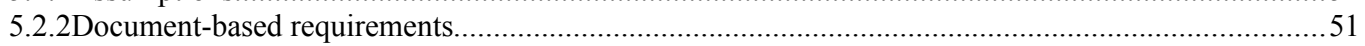

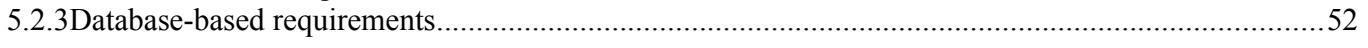

6.OBSOLETE SOFTWARE REQUIREMENTS EFFECTS .........................................................56

6.1OBSOLETE SOFTWARE REQUIREMENTS EFFECTS ON THE PROJECT SIZE................................................56

6.2OBSOLETE SOFTWARE REQUIREMENTS EFFECTS DEPENDING ON THE TYPE OF THE PROJECT.......................58

7.THE REQUIREMENTS ENGINEERING PROCESS AND OBSOLETE SOFTWARE

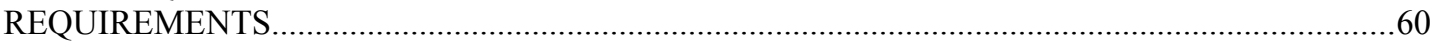

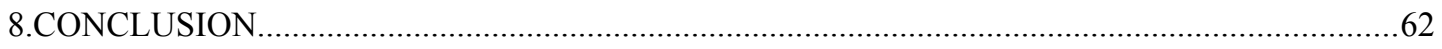

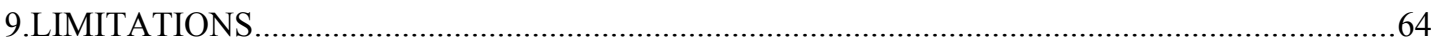

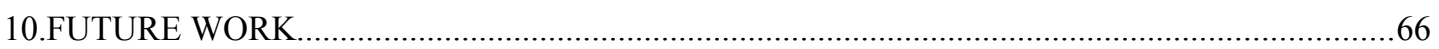

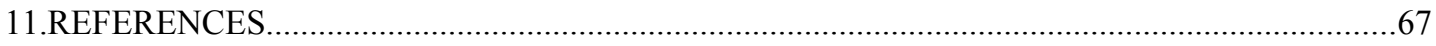

APPENDIX A: OBSOLETE SOFTWARE REQUIREMENTS QUESTIONNAIRE.........................71 


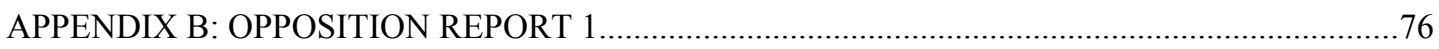

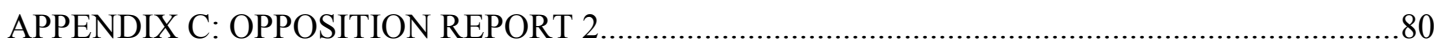




\section{INTRODUCTION}

Requirements Engineering (RE) is deemed to be one of the most important phases of any Software Project Life-Cycle (SPLC). The success of any software project depends heavily on high quality requirements, and on requirements engineering process [1], [2]. Aurum and Wohlin emphasized that "managing the RE phases is vital to the successful development of software products" [3].

According to Sommerville, requirements management is "the process of managing changes to the system requirements" [1]. It mainly consists of requirements changes, requirements status, requirements tracking, and requirements traceability [2]. As well, it is usually done concurrently with other RE activities. Moreover, he advises that "the process of requirements management should start as soon as a draft version of the requirements document is available" [1].

On the other hand, it is essential to have requirements management as an integral part of the RE process. A successful requirements management process ensures the manageability of the requirements of the product throughout the SPLC, and it also ensures that the developed product is what was asked by the customer [2]. Additionally, there are several ways of managing requirements. One common approach is the use of excel sheets for managing small or medium sets of requirements (less than 1000 requirements [4]). However, a large and very large volume of requirements require a systematic approach supported by requirements management tools.

Undoubtedly, changes of requirements are unavoidable in any software project. Requirements change over time as software projects progress. As well, involved stakeholders (mainly customers) and developers gain better understanding of the final product. The amount of changes grows significantly in case of large projects. Combined with the often inevitable and constant stream of new requirements and customer requests, that amount can cause information overload, and can severely impede the requirements management process [4]. In the United States, for example, requirements creep at a monthly average of $2 \%$ [5]. Besides, latest researches have shown that requirements changes have been one of the most common software weaknesses between the years 2000 and 2008 [5]. This evidence confirms the results of the Chaos Report by the Standish Group that changing requirements present a challenge to the success of software projects [6].

Changes in software requirements make them volatile. Requirements volatility is a common phenomenon in software projects. Moreover, it is believed depending on the size of the project, that between $30 \%$ to $50 \%$ of software requirements of any project are volatile [7]. Studies have shown that requirements volatility affects the quality of software projects, and imposes several risks ranging from causing delays in the delivery of the software projects and its maintenance, increasing the density of defects, and leading to economic consequences [7-11]. These risks are believed to incur due to lacking practices relevant to requirements volatility in the requirements engineering process [12]. In addition to, volatile requirements have some influences on decisionmakers on deciding what software requirements to include/exclude in a project or a release [9].

Furthermore, time and budget constraints prevent implementing all candidate requirements and force project management to select a subset of requirements that are prioritized more important than the others so as to be implemented [9], [13]. As a result, some requirements become cancelled and deleted during the elicitation and specification phases while other requirements are considered not important during the prioritization phase. A common scenario in this situation is to leave the excluded requirements for being considered in the next release. The constant leaving of the 
excluded requirements for the next release may simply render them obsolete. To illustrate, according to Murphy and Rooney "requirements have a shelf life: the longer the time between gathering and implementation, the greater the risk impact of change. Change makes requirements obsolete" [14].

Moreover, a recent survey of how documentation is used by practitioners during the course of software projects reports that documentation is usually outdated. Also, the same survey reports that the final software product does not mostly meet its documentation [15]. Consequently, the documentation still holds outdated or obsolete requirements, or even entire requirements specifications that were not implemented or changed over time. The importance of documentation in many projects should not be neglected because it forms the contract between the software customer and supplier (developer) [16].

In the same way, continuous changes in requirements, documentation that contains out-of-date information, new incoming requirements monthly, and other problems in requirements, all of these create burdens on many stakeholders by causing "information overload" [13] especially at large and very large-scale projects. Similarly, OSRs will contribute to the the problem of information overload. According to Natt and Gervasi, the result is that "the requirements management process easily becomes overloaded due to the sheer number of requirements" [13].

The focus of this master thesis is on obsolete software requirements (OSRs). The concept of OSRs seems not to have any sensible interest in the research area. Therefore, there is no definition in the literature of what OSRs are, or how a requirement becomes obsolete, or how to manage them, or when to check for OSRs. Additionally, it is not known whether OSRs present any challenges to software projects. In case OSRs have negative effects on software projects, these effects might impede the projects and compromise their quality.

There is a paucity of information in the literature about OSRs (see Section 3.3 Obsolete software requirements in the literature). Thus, looking up the word "obsolete" from the English dictionary would help us better understand (but not necessarily) what we are talking about. According to Cambridge Dictionaries Online obsolete means "not in use any more, having been replaced by something newer and better or more fashionable"[17]. Only few articles mentioned the term "obsolete requirements" or talked about obsolescence of software requirements in general. They only specified that some requirements become obsolete, and they are supposed to be marked as such, or be included in the documentation for future reference [18], [19]. Anyway, in their studies of requirements volatility, Nurmuliani, Zowghi, and Powell mentioned the term "obsolete functionality". They defined it as: "functionality that no longer required for the current release or has no value for the potential users" [20].

On the other hand, there are studies that talk about obsolescence of software and hardware components or artefacts. For instance, Merola says "software applications become obsolete when they are retired from use and taken off the market due to technology advancements, decrease in product popularity, or other market factors. Vendors may no longer sell or support licenses for older versions of the application" [21]. This, however, is different from OSRs (see Section 3.3.2).

In essence, these instances in the literature give a clue about OSRs. Nevertheless, they do not form strong evidence about the presence of OSRs neither in practice nor in the literature. This master thesis addresses and investigates the phenomenon of OSRs from both practical and literature perspectives. The major objectives of this master thesis are: to define what OSRs are, to find the relationships between OSRs and requirements types, to propose processes to discover OSRs and to take possible actions against them when they are discovered, and to study the effects of OSRs.

In order to find information about OSRs, the author searched the literature for evidence about OSRs through the Systematic Literature Review (SLR). Moreover, a 
web-based questionnaire was distributed to potential respondents over the Internet using social networks and emails. The questionnaire aimed to gather evidence from the industry and academia about OSRs.

The rest of this master thesis report is organized as follows:

- Section 2 describes the research methodologies followed in this master thesis, and it presents some results of the questionnaire (i.e. demographic results).

- Section 3 defines OSRs and examines its existence and seriousness.

- Section 4 develops a classification of requirements based on the volatility requirements notion. As well, section 4 provides basic relationships between OSRs and types of requirements.

- In section 5, the author describes possible ways/processes to find or detect OSRs in the requirements documentation. Moreover, he suggests some actions to be taken against OSRs after they are discovered.

- Section 6 studies the effects of OSRs on the project's size, and on the project's type.

- In section 7, OSRs are connected to the whole process of requirements engineering and requirements management processes.

- Finally, the conclusion of the master thesis, its limitations, and future works are presented in section 8 , section 9 and section 10 , respectively. 


\section{Research Methodology}

There are many research methodologies that can be used to embark on a research. However, Software Engineering research can be conducted using several research methodologies namely: controlled experiments, case studies (both exploratory and confirmatory), survey research, ethnography, and action research [22]. These methodologies are mainly connected with social sciences because software engineering is a multi-disciplinary field [22]. Additionally, literature review is a very common methodology in almost all fields which aims at gathering evidence from previous researches reported in the literature. Nevertheless, due to the nature of software engineering discipline, Kitchenham proposed a Systematic Literature Review (SLR) [23] methodology in order to search the literature for evidence about topics in software engineering. This master thesis is beyond discussing the differences among research methodologies, or how and when they are used. For further information about them, the reader can refer to [22-24]. However, the following paragraphs briefly describe the research methodologies followed in this master thesis.

Systematic Literature Review (SLR): Kitchenham defines SLR as "a means of identifying, evaluating, and interpreting all available research relevant to a particular research question, or topic area, or phenomenon of interest" [23]. According to Kitchenham [23], the goals of SLR are: to summarize existing evidence concerning a research topic, to identify any gaps in the current research areas in order to allow researchers to investigate these gaps and fill them, and to "provide a framework/ background in order to appropriately position new research activities"[23]. In this master thesis, the goal of using SLR (see section 2.1) intersects with the mentioned goals of the SLR. However, the outcome of the SLR was not helpful in answering the research questions of this master thesis concerning OSRs.

Survey research (questionnaire): survey research is "used to identify the characteristics of a broad population of individuals" [22] about certain topics. Survey research can be conducted in the forms of interviews (structured, unstructured, and semi-structured), data logging techniques, or questionnaire (paper based, web based, and phone based). The major goal of survey research is to collect data from the representatives of the population about the studied topic [22], [24]. In this master thesis, web based questionnaire (see section 2.2) is used to gather information from experts in the industry and academia concerning OSRs. As well, the use of the questionnaire is to complement the outcome of the SLR in answering the research questions of this master thesis. On the other hand, the use of web based questionnaire was due to its advantages such as cheapness and targeting more audience. To illustrate, the questionnaire was distributed to potential respondents over the Internet utilizing emails and social networks.

The rest of this section describes in details the usage of SLR (section 2.1) and web based questionnaire (section 2.2). As well, section 2.3 describes briefly some statistical methods that were used in analyzing the results of the web questionnaire.

\subsection{Systematic Literature Review}

A systematic Literature Review (SLR) was conducted during the master thesis. The SLR in this thesis follows the recommendation of Kitchenham [23] and is inspired by another SLR done by Unterkalmsteiner et al. [25]. The reason behind using [25] is that it follows Kitchenham's guidelines [23], and it presents a good and recent example of conducting SLR that complements Kitchenham's guidelines.

The steps of conducting SLR are depicted in Figure 1, and detailed throughout this section. In this SLR, however, not all of the steps were followed. Accordingly, that will be reported. 
Before SLR, a preliminary literature search for information about OSRs was conducted by using the following search string:

"((obsolete OR outdated OR deprecated OR obsolescence) AND (requirements OR specifications))".

The search was executed on several databases namely Inspec/Compendex, ISI Web of Science, Scopus, IEEE, ACM digital library, SpringerLink, and Google scholar. It was scoped on the fields of title, abstract, and keywords. In addition to, there was no date limit on the search.

The preliminary literature search yielded inadequate results about obsolescence of requirements in the context of software engineering and/or requirements engineering. Due to that, the need for SLR (step 1 in Figure 1) emerged in order to collect information and facts about obsolescence of software artefacts, in general, and about obsolescence of software requirements, in particular, in the context of software engineering and requirements engineering, and, to find answers for the research questions (step 2 in Figure 1) of this master thesis (see Table 1).

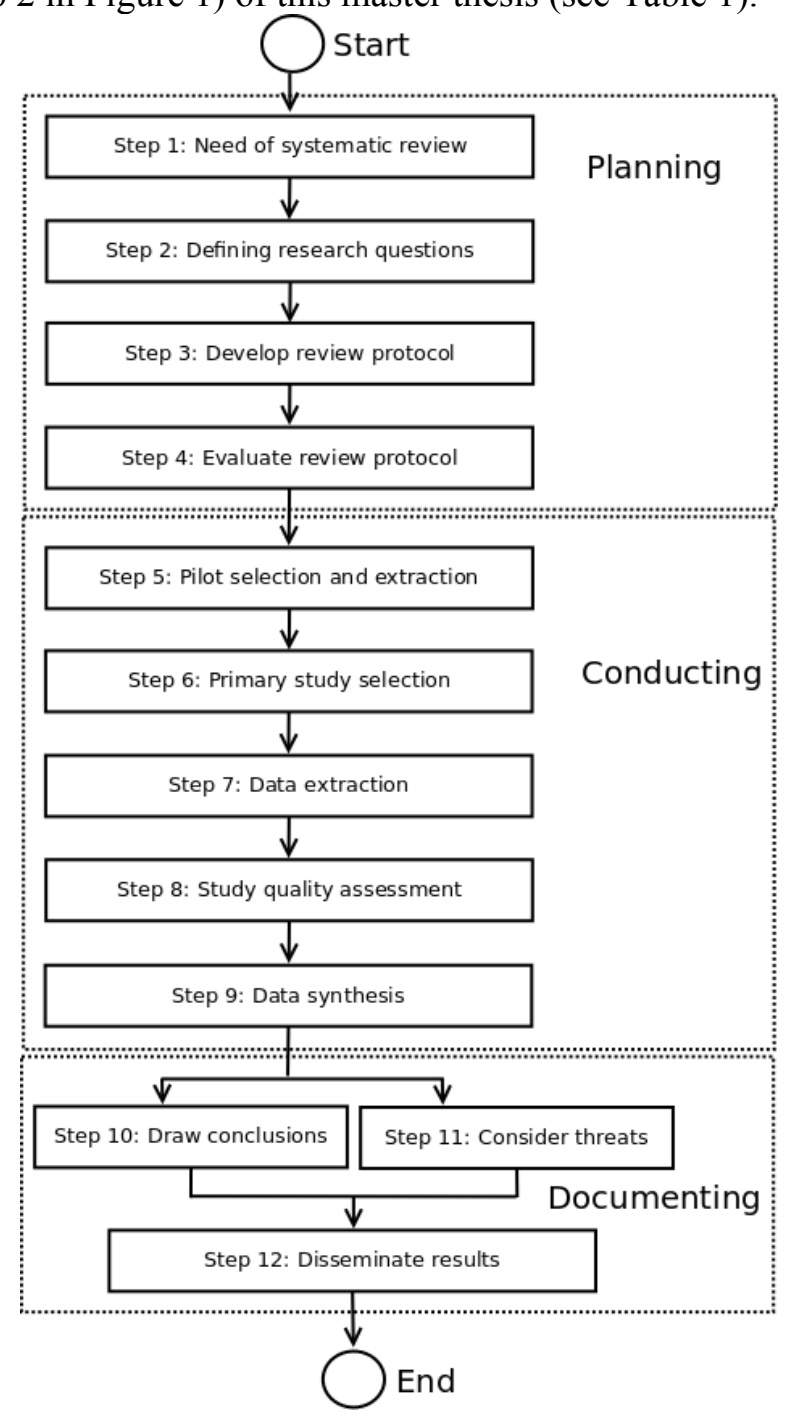

Figure 1: Systematic literature review steps, adapted from [19] 
Table 1: Research questions of the thesis

\begin{tabular}{|c|c|c|}
\hline \# & Research Question & Aim \\
\hline 1. & $\begin{array}{l}\text { Does the phenomenon of obsolete } \\
\text { software requirements exist? }\end{array}$ & $\begin{array}{l}\text { To investigate existence of obsolete } \\
\text { software requirements phenomenon in } \\
\text { literature and industry. }\end{array}$ \\
\hline 2 & $\begin{array}{l}\text { How could an obsolete software } \\
\text { requirement be defined? }\end{array}$ & $\begin{array}{l}\text { To propose the definition of obsolete } \\
\text { software requirements and its initial } \\
\text { validation in an example of requirements } \\
\text { engineering context. }\end{array}$ \\
\hline 3 & $\begin{array}{l}\text { What are the types of requirements } \\
\text { that are eligible to become obsolete? } \\
3.1 \text {. What is/are the possible } \\
\text { process(es) to discover/find/expose/ } \\
\text { detect/mark the obsolete software } \\
\text { requirements? }\end{array}$ & $\begin{array}{l}\text { To classify requirements types and their } \\
\text { relationships to obsolescence of } \\
\text { requirements. } \\
\text { To enact a process to detect/identify/find } \\
\text { obsolete software requirements or } \\
\text { nominate requirements that will later } \\
\text { become obsolete. }\end{array}$ \\
\hline 4 & $\begin{array}{l}\text { When obsolete software requirements } \\
\text { are defined/identified/discovered, } \\
\text { what is/are the possible action(s) to } \\
\text { take against them (e.g. discard them, } \\
\text { keep them)? } \\
4.1 \text {. What are the possible actions to } \\
\text { take in a database of requirements? } \\
4.2 \text { What are the possible actions to } \\
\text { take in a specification/requirements } \\
\text { document? }\end{array}$ & $\begin{array}{l}\text { To suggest actions to be taken against } \\
\text { obsolete software requirements. For } \\
\text { instance, discarding the obsolete } \\
\text { requirements from the } \\
\text { documentation/database, or moving } \\
\text { them into a separated section, or leaving } \\
\text { them as they are. } \\
\text { To suggest a change in the format of the } \\
\text { IEEE Software Requirements } \\
\text { Specification SRS document, or other } \\
\text { requirements specification documents in } \\
\text { order to include or consider obsolete } \\
\text { software requirements. }\end{array}$ \\
\hline 5 & $\begin{array}{l}\text { What context factors, such as project } \\
\text { size or domain/context that the project } \\
\text { is running, the obsolete software } \\
\text { requirements do affect? }\end{array}$ & $\begin{array}{l}\text { To find the context factors, such as } \\
\text { project size or domain/context that the } \\
\text { project is running, which obsolete } \\
\text { requirements affects. }\end{array}$ \\
\hline 6 & $\begin{array}{l}\text { Where do obsolete software } \\
\text { requirements exactly fit in the } \\
\text { requirements engineering life cycle? } \\
\text { e.g. in requirements traceability, } \\
\text { change requirements, requirements } \\
\text { volatility, etc. }\end{array}$ & $\begin{array}{l}\text { To locate/position requirements } \\
\text { obsolescence in the requirements } \\
\text { engineering life cycle. }\end{array}$ \\
\hline 7 & $\begin{array}{l}\text { Does the industry have any practice(s) } \\
\text { or process(es) regarding obsolete } \\
\text { software requirements? What are } \\
\text { they? }\end{array}$ & $\begin{array}{l}\text { To investigate the industry for the } \\
\text { industrial practices regarding all the } \\
\text { above mentioned points. }\end{array}$ \\
\hline
\end{tabular}

The review protocol (step 3 in Figure 1) was developed so as to discover relevant information about the research questions in general, and "to reduce potential researcher bias and to permit a replication of the review in the future" [25]. The protocol experienced the evaluation of the three supervisors of this master thesis who are experts in conducting researches in software engineering. Based on their feedback 
and insights (step 4 in Figure 1) the protocol was then improved. Well, this review protocol is described in section 2.1.1 to 2.1.5.

\subsubsection{Search Strategy}

Since the preliminary literature search (mentioned in section 2.1) did not produce results to answer the research questions of the master thesis (see Table 1), the keywords in the search string needed to be expanded in order to obtain more results about OSRs. Therefore, the search string was formulated from synonyms of obsolete and its variants (see 3.1 Meaning and Synonyms), and keywords that are related to requirements or used by researchers to mean or refer to requirements. The final search string for the SLR is:

(obsolete OR obsolescent OR obsolescence OR outdated OR out-of-date OR deprecated) AND (requirement* OR specification* OR functionalit* OR feature* OR component*) AND (software).

The inclusion of the word "software" in the search string is necessary to exclude the results related to hardware, avionics, military devices, and electronics. The pilot search included many results related to them and these results are outside the scope of the thesis. Therefore, a decision was taken to reduce the number of irrelevant results by including the word "software" in the search string.

The search was run on several databases namely: Inspec/Compendex (Engineering Village), ISI Web of Science, Scopus, ACM, and IEEE. It was planned to use SpringerLink, but due to technical limitations from SpringerLink side, the search was not conducted. The problem is that SpringerLink does not accept more than 10 search terms meanwhile the search string (showed above) contains 12 terms.

\subsubsection{Study Selection Criteria}

Despite the recommendations of Kitchenham [23] to limit the search for publications within the last decade, this SLR search was done between the years 1990 and 2011. The reason behind extending the search time-frame was to include more results related to the topic that might have been discussed in the past since there is no recent or any study that is dedicated to OSRs or talks about them -to the best of my knowledge-.

The search included conference articles, conference proceedings, journal articles, posters, workshop articles, and reports. Moreover, the language of the search was only limited to English. Finally, the search was conducted on the meta-data fields i.e. title, abstract, and keywords only.

The search was scoped to topics related to computer science and software engineering in some databases. The need for the scoping was necessary to eliminate enormous amount of irrelevant results from other realms of knowledge, because the search string contains general keywords that are used by other fields. To illustrate, on Scopus database, the search was restricted to the physical sciences that include computer science. As well, in ISI web of Science database, the search was scoped to the subject areas of computer science, software engineering, and information technology. Finally, in ACM database, the search was conducted only on the fields of title and abstract, because ACM does not support the field of keywords.

\subsubsection{Study Selection Procedures}

In order to decide how to select relevant articles, a pilot study (step 5 in Figure 1) was conducted on Inspec/Compendex (Engineering Village). The pilot study yielded no results (see Section 2.1). As a result, a refinement of the search string was done to include more keywords that could obtain results related to obsolescence of requirements. Nevertheless, the selection criteria that were used during the pilot study are the same for the rest of the study and are explained below:

1) Read the title: 
- If the title includes at least one word from the search string, and the title indicates that the article is related to software engineering topics, then, the article will go to step 2 .

- If the title does not mention any of the keywords specified in the search string, then, the article is rejected.

2) Read the abstract: the abstracts of all the articles that came from step 1 will be read. If any abstract shows that the topic of the article is related to obsolescence of software, or to any software artefacts (e.g requirements), then, the article will be included into step 3. Otherwise, it will be rejected.

3) Read the article: the complete text (title, abstract, and full text) of all the articles that came from step 2 will be read. If the article talks about obsolescence of software or is related to it or any other software artefacts (e.g requirements), then, the article will go to the "study quality assessment" (see section 2.1.5).

Figure 2 shows the number of retrieved articles from each database (step 6 in Figure 1). From 116 articles chosen to be relevant based on reading their titles (step 1), 53 articles were duplicated among the databases, and 63 articles went to the next step i.e. abstract reading (step 2). After reading the abstracts, 38 articles were rejected at this stage because they do not discuss issues related to obsolescence of software requirements. Finally, 25 articles were read thoroughly (step 3). Only three of these were identified to be partially relevant to OSRs and they are [26-28] (see section 3.3.1 The results of the systematic literature review).

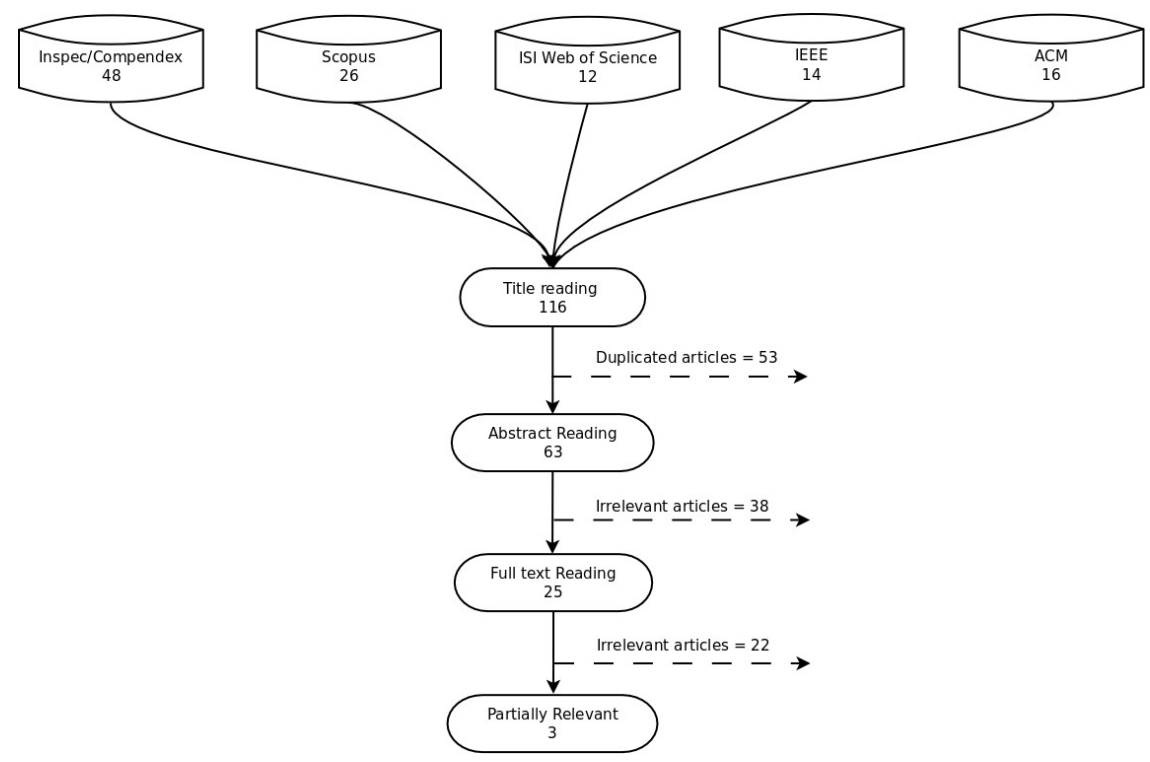

Figure 2: Studies Selection

\subsubsection{Data extraction}

As shown in Figure 2, the SLR yielded only three articles that are partially related to OSRs. These articles discuss different topics in which OSRs are mentioned in them as a sentence or idea. Therefore, there is no need for any data extraction mechanism (step 7 in Figure 1) for this small amount of articles.

\subsubsection{Study Quality Assessment}

Despite specifying the criteria of the quality assessment step of the study beforehand (step 8 in Figure 1), it turned out that this step was not conducted due to 
only three accepted papers. However, for the sake of reporting the criteria of the study quality assessment, below are their description:

1) Does the article address issues in management of software artefacts?

2) Does the article address matters related to software requirements?

3) Does the article talk about obsolete, deprecated, or outdated software artefacts, in general?

4) Does the article talk about obsolete, deprecated, or outdated software requirements, in particular?

5) Is the work of the article empirical?

6) Are the objectives and aims of the study well defined?

7) Are the findings clearly stated, and capable of fulfilling the defined objectives?

\subsubsection{Data synthesis}

Data synthesis (step 9 in Figure 1) is inapplicable for this SLR due to the small number of accepted articles.

\subsubsection{Documenting the results of the SLR}

The last stage of the SLR study is to document its findings i.e. step 10, 11, and 12 in Figure 1. Since the number of accepted articles in this SLR was only 3 articles, the results will be documented in section 3.3.

\subsection{Survey - Web Questionnaire}

The overall objective of the web questionnaire is to find empirical answers to the research questions (see Table 1) and to collect data from the academia and industry experts about OSRs.

The choice of a web-based questionnaire was due to the advantages it possesses over paper based questionnaire or interviews. Automation of data collection and analysis, and flexibility and convenience to both the researchers and the participants, targeting easily more audience, ease of access, and cheapness [24], are only some advantages.

\subsubsection{Preparation of the questionnaire}

The content of the questionnaire was derived from relevant topics of OSRs such as volatility requirements, change management, requirements traceability, and requirements management in general. Moreover, another source of input to the questionnaire was the results of the SLR (presented in section 3.3.1). However, the contributions of SLR results to the questionnaire were really subtle because the SLR did not produce any solid evidence about OSRs. Therefore, the author had to suggest the questions and possible answers to them. Later on, the content of the questionnaire was discussed with the three supervisors of the thesis in a manner such that their comments were considered to improve it. The questionnaire went through several amendments until it was finalized and published. The final version of the web questionnaire is available at Appendix A.

The questionnaire consists of 15 open and closed questions of different formats i.e. multiple choice questions (one answer only or multiple answers). Regarding the open questions, the respondents have the possibility to provide their own answer in case they are not listed amongst the choices. As well, respecting some of the closed questions, an ordinal scale from 1 to 5 where 1 is 'not likely' and 5 is 'very likely' was used. 
Furthermore, the questionnaire comprises of two main parts. The first part asks questions related to OSRs. As mentioned earlier, OSRs questions were formulated by the author and then refined after the supervisors' comments. A mapping between the questions in the questionnaire and the research questions in Table 1 is done at the end of Appendix A. The second part of the questionnaire was designed to collect demographical information about the participants such as the role of the participant (e.g. project manager), the job/business domain (e.g. telecommunication), the development methodology followed (e.g. waterfall), the duration of the projects, and the types of requirements engineering (e.g. outsourced projects).

The following paragraphs describe how the questions and answers of the demographic part of the questionnaire were formulated (see Appendix A).

Roles of respondents in their respective organizations: the targeted audience is working on different roles related to software engineering or software development life-cycle. A list of possible roles in software engineering was synthesized from [29], [30]. The question is an open-closed one where the respondents can either choose a predefined role or specify another role that is not listed (only one choice).

Types of businesses or domains of respondents: many software organizations develop applications for government agencies, hospitals, universities, banks, and grocery stores, to name a few. It is often that software organizations use or adopt different development methodologies from domain to domain. Therefore, the knowledge about the domain is important to know the domains that the respondents are working on. Moreover, it gives the results of the questionnaire more credibility especially, if the results to be generalized. Doubtlessly, the list of domains could be endless, thus, a list of 15 domains were adapted from [31]. Additionally, the respondents have the ability to choose more than one domain they have been working on or even specify another domain that is not mentioned in the list (multiple choice).

Size of the organizations: the size of organization that the respondents work at is important for analyzing the results. There is no agreement on classification of organizations' sizes in the literature. For instance, small organization has less than 10 employees or 50 employees. Therefore, the classification that is followed in this questionnaire has no names for the categories and it only uses the ranges of number of employees in organizations. For this purpose, seven categories of organizations' sizes were predefined: less than 10 employees, $11-20$ employees, 21 - 50 employees, 51 100 employees, $101-200$ employees, $201-500$ employees, and over 501 employees. This list was inspired by [32]. The respondents can only choose one category of them (single choice).

Average duration of projects: the duration of projects contributes to getting requirements obsolete. Short duration projects are less likely to have OSRs especially, after the deployment, because they tend to be small in number of requirements, easily managed, and their life-cycle might be short. On the other side, projects with relatively longer duration are more likely to have OSRs because such projects might go through several iterations and changes in requirements. There is no standard definition of project duration as this normally varies from project to project. However, the respondents are asked about the average duration of projects they were involved in; as many people would be involved in several projects having a variety of durations. Here, the division of durations is as follows: $0-3$ months, $4-6$ months, $7-12$ months, $13-$ 24 months, 25 - 36 months, and over 37 months. The respondents can only choose one answer (single choice).

Development methodologies and processes: there are several development methodologies followed by software organizations in order to build software. The way the requirements are gathered and managed differs based on the development methodology. For instance, in waterfall, changes in requirements are not welcomed during project implementation. This is unlike agile development whereby changes are 
welcomed at anytime during the course of the project. It is possible that an experienced software developer or manager has used more than one development methodology throughout his/her career. Therefore, the respondents are allowed to choose more than one development methodology (multiple choice), as well as to specify others in case the methodology is not listed. The list of development methodologies and processes were derived from [32], [33].

Types of requirements engineering: the stakeholders' types determine to some extent the type of the requirements engineering methodologies to be followed. To illustrate, the way requirements are gathered, elicited, specified, validated, and managed differs from bespoke projects (projects that are made for specific organizations) to market-driven projects (projects that are made for the open market place) to outsourced projects (software that is given to a third party to produce it) to open source projects.

\subsubsection{Data collection}

A system website support i.e. SurveyMonkey.com was used for this questionnaire because of its reputation, and the features it provided for the questionnaire. Besides, it is very convenient for both the author and the respondents as it is web-based.

Invitations to participate in the questionnaire were sent to potential audience via emails or a social network website i.e. www.linkedin.com. The use of social networks increases the number of potential respondents. The strategy followed to target potential respondents was to search for software engineering or requirements engineering groups and companies on "Linkedin.com", and then to post the questionnaire's link on the board of the group or the discussion area. In addition to, emails to individuals were sent in these groups based on their designated titles e.g. senior software engineer, requirements engineer, system analyst, project managers, only to name a few. At this step, master students and undergraduate students were avoided because their experience could have been insufficient in order to answer the questionnaire.

Software companies and requirements management tools' producers were targeted as well, because they possess the required knowledge and experience about OSRs (these names and addresses were acquired from the references of [34], and from lists of requirements management tools via Google search).

Furthermore, all participants were guaranteed anonymity of their particulars and answers. Additionally, all the information (answers of the questionnaire) were automatically stored by SurveyMonkey.com.

The questionnaire was published online on $3^{\text {rd }}$ of April 2011 and was switched off after 30 days on $3^{\text {rd }}$ of May 2011. In total, about 1700 individual invitations were sent to people either on Linkedin or by emails, and about 10 posts on requirements engineering and software engineering groups' discussion boards were posted. The number of collected responses is 223 , and only four of them were deleted because the respondents did not fill the demographic section. In total, 219 responses are analyzed.

\subsubsection{Results of the web questionnaire (demographic part)}

In this section, parts of web questionnaire results will be presented namely the demographic questions. Whereas, the results of other questions related to OSRs will be reported in the rest of the thesis report according to their suitability to the sections.

\section{Respondents' residence country}

Surveymonkey.com system automatically collects the IP addresses of the respondents (usually this IP address is the ISP's address of the respondent and not the actual respondent's IP address). Using tools which determine the country (location) of the IP address, it is useful to know where the respondents answered the questionnaire and it is most likely where they come from. On the other hand, this is not considered tracking the respondents as it does not identify them personally. 
The use of Linkedin.com allowed for targeting more audience from all over the world. People from 45 countries and all the continents have responded to the questionnaire. Figure 3 shows only the participants' top countries while the rest are presented in Table 2. Responses from the United States were the highest with 40 responses followed by the United Kingdom with 28 responses. Sweden has come in the third place with 25 responses followed by Netherlands (18 responses), Canada (12 responses), Germany (11 responses), India (8 responses), Australia (7 responses), and Malaysia (5 responses). The list of all participants' countries is shown in Table 2 along with the number of participants from each country.

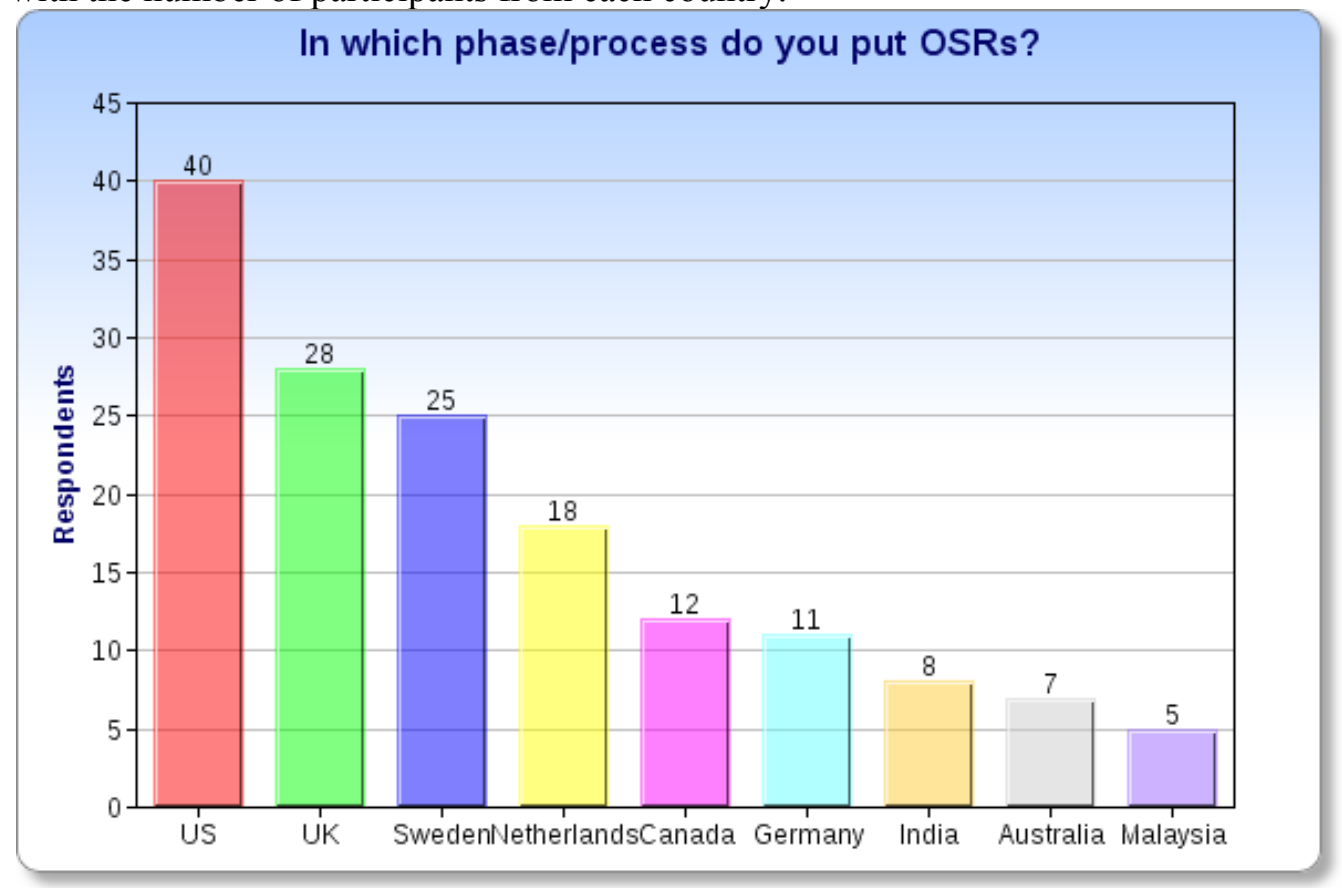

Figure 3: Top countries of respondents

Table 2: List of participants' countries 


\begin{tabular}{|l|l|l|l|l|l|}
\hline Name & Count & Name & Count & Name & Count \\
\hline US & 40 & Romania & 3 & Ireland & 1 \\
\hline UK & 28 & South Africa & 3 & Jordan & 1 \\
\hline Sweden & 25 & Brazil & 2 & Kenya & 1 \\
\hline Netherlands & 18 & China & 2 & Kyrgyzstan & 1 \\
\hline Canada & 12 & Egypt & 2 & Lebanon & 1 \\
\hline Germany & 11 & France & 2 & Mexico & 1 \\
\hline India & 8 & Greece & 2 & New Zealand & 1 \\
\hline Australia & 7 & Italy & 2 & Norway & 1 \\
\hline Malaysia & 5 & Pakistan & 2 & Poland & 1 \\
\hline Argentina & 4 & Switzerland & 2 & Portugal & 1 \\
\hline Belgium & 4 & Austria & 1 & Saudi Arabia & 1 \\
\hline Finland & 4 & Bulgaria & 1 & Singapore & 1 \\
\hline Russia & 4 & Chile & 1 & UAE & 1 \\
\hline Spain & 4 & Costa rica & 1 & Ukraine & 1 \\
\hline Turkey & 3 & Croatia & 1 & Japan & 1 \\
\hline
\end{tabular}

\section{Roles of respondents in their respective organizations.}

The question is an open-closed question where the respondents can either choose a predefined role or specify another role that is not listed (only one choice), (see question 10 in Appendix A). Some of the respondents have written down roles in the "others" category that are either mentioned in the list or form parts of some roles. To illustrate, this description "requirements management covering both software and non software requirements" was moved into requirements engineers/specialists because requirements management could be part of the requirements managers tasks. Another example, " 70 percent time testing and 30 percent time usability \& design" was moved into software tester because this person spends most of his time in testing. In total, 18 responses were moved into predefined roles, and 30 responses were analyzed (see next paragraph).

It is clear from Figure 4 that the respondents come from different roles in their respective organizations. Nevertheless, about one quarter of the respondents (24.9\%) are requirements engineers/specialists, followed by researchers/academicians from the software engineering fields (11.5\%). Software architects/designers and software project managers come in the third place with a score of $10.1 \%$ each. Then come software team leaders (8.3\%), developers/programmers/coders $(7.8 \%)$, software product manager and software testers/test designers $(5.5 \%$ each), software integrator $(1.4 \%)$ and software maintainer $(0.9 \%)$. We can assume that people in these roles work closely with requirements and requirements management more than others. This gives the results of the questionnaire extra credibility and allows for the generalization of results. 


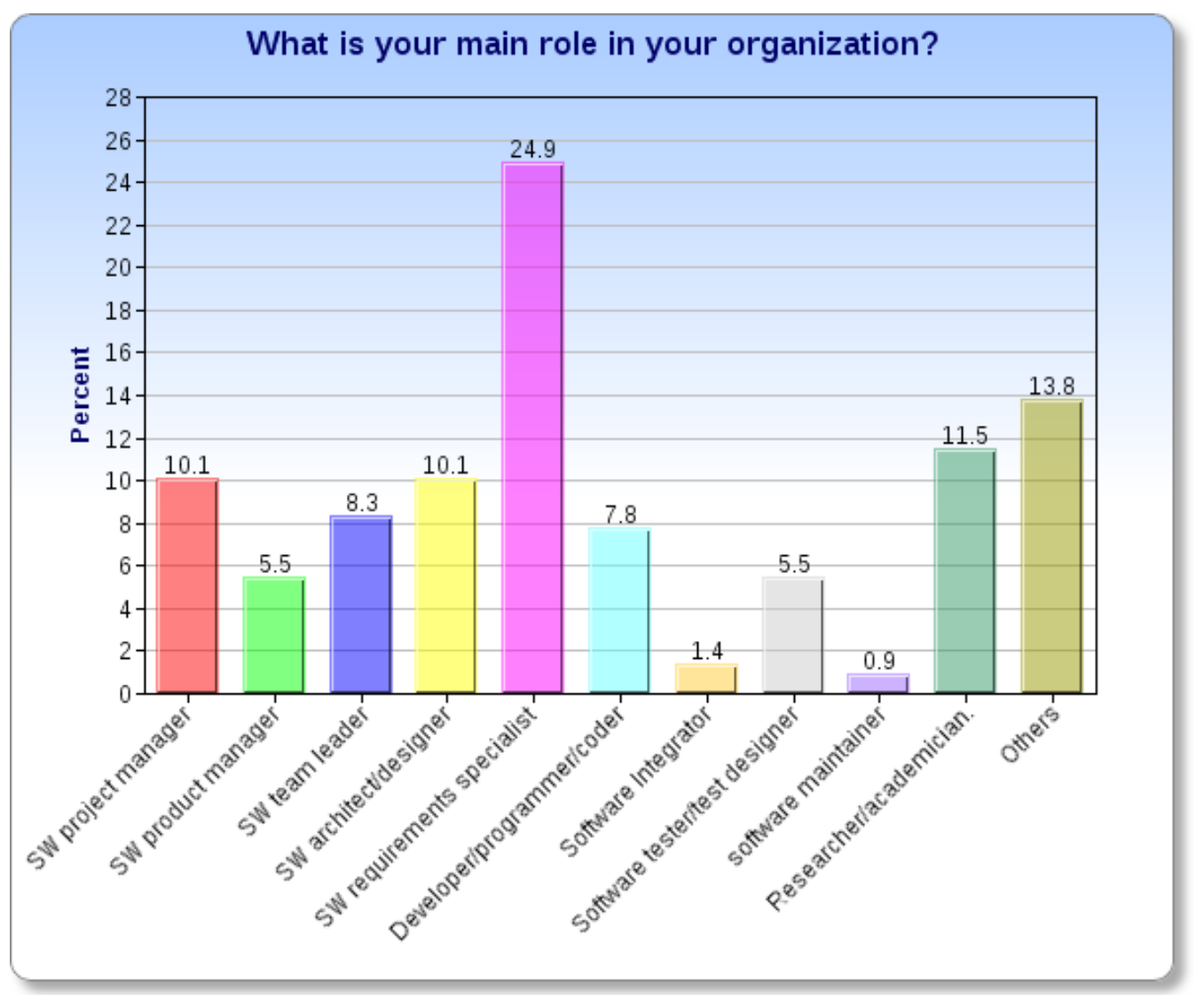

Figure 4: Respondents roles

It is important to know here that in the "others" categories, people came from different roles and they are: CEO (1 person), Vice president (1 person), system engineers ( 3 people), software quality assurance ( 3 people), process engineers (4 people), business analysts (4 people), software engineers that are involved in many SPLC phases (4 people), consultants (5 people), and managerial roles ( 5 people).

The total number of respondents to this question is 217 out of 219 . So, 2 people did not answer this question.

\section{Types of businesses or domains of respondents}

The respondents have the ability to choose more than one domain they have been working on or even to specify other domains that are not mentioned in the list (multiple choice), (see question 11 in Appendix A).

The respondents of the questionnaire are coming from all the predefined domains with different percentages. In addition to, some respondents had specified other domains that are not listed. The reported results (below) are computed based on the number of responses received for every category. Since the respondent is allowed to choose more than one category, the computation is done over the number of responses and not over the number of respondents (as in person).

In general, Figure 5 shows that the majority of respondents $(31.9 \%)$ work in domains of information technology, computer, and software services. The second largest group works in different engineering fields such as automotive, aerospace, power, and others (only 12.5\%). These were followed by telecommunication (10.6\%), consultancy $(9.1 \%)$, government and military applications (7.5\%), banking and finance $(6 \%)$, health/medical and education $(4.9 \%$ each), and the rest of the domains are shown in Figure 5. 


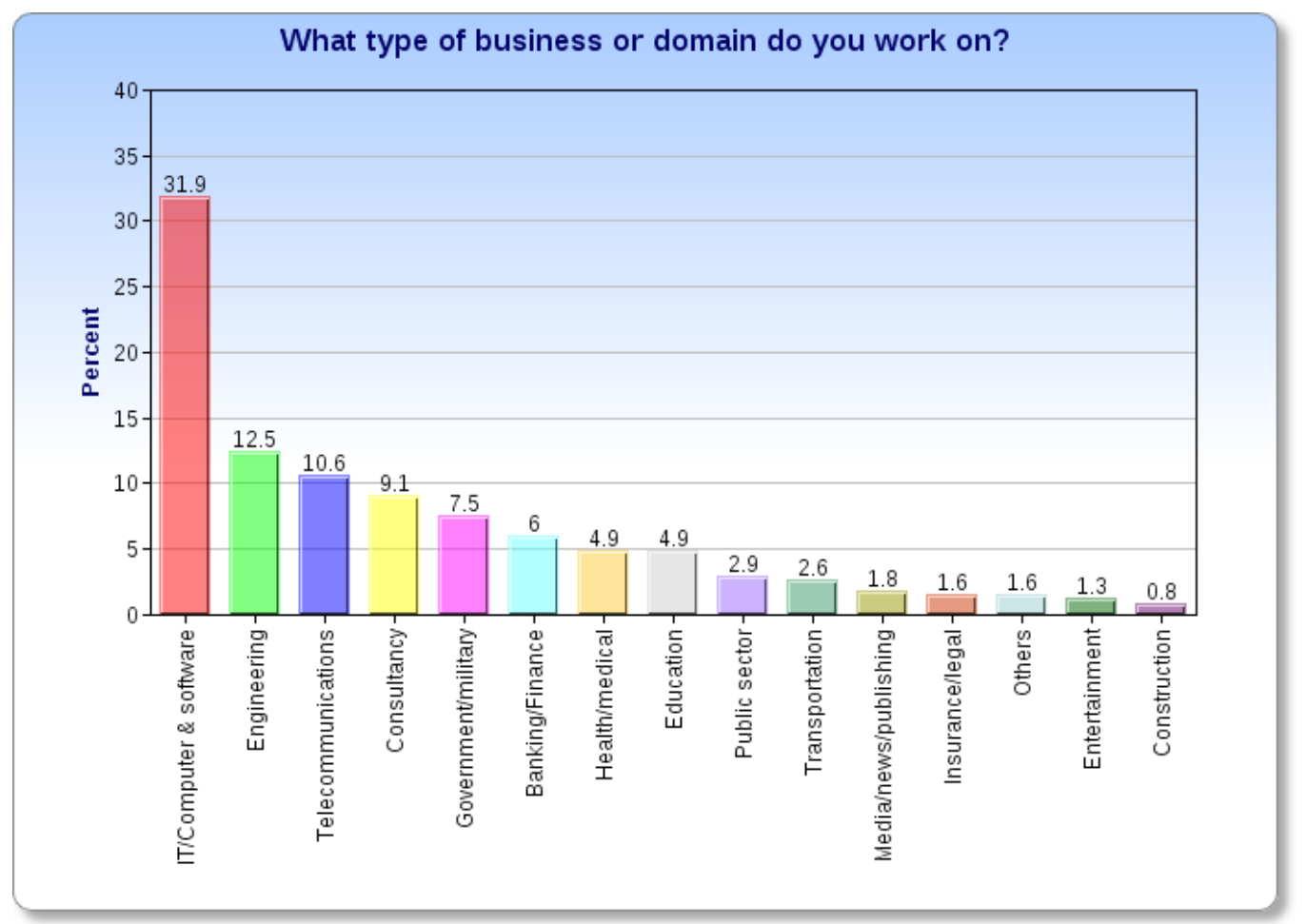

Figure 5: Respondents businesses or domains

Several responses went to the "others" category in which people wrote down other domains they work on. In fact, some of the answers can be integrated in the predefined list of domains, or they were just repeated by the respondents in order to be more specific. However, the author moved 12 of them into their respective categories. After that, "others" category comprises of 6 responses (1.6\%) and they are: research, food creation processes and ornamentals, simulation \& training systems, security, distribution, and broadcast. Moreover, only 1 person skipped this question and did not answer it.

\section{Size of the organizations}

The respondent can only choose one category of predefined categories of sizes (single choice), (See question 12 in Appendix A).

According to Figure 6 more than half of the respondents (53.7\%) came from large organizations i.e. 'over 501 employees', followed by the smallest category 'less than 10 employees' with only $12.5 \%$ of respondents. Note here that there is a big gap between the answers, mainly between 'over 501 employees' and the rest of them. The rest of the choices received less than $8.5 \%$ of responses as shown in Figure 6 . Only $5.1 \%$ of respondents are coming from organizations of '11-20 employees', $6 \%$ of employees are coming from '51 - 100 employees' organizations, 15 representatives of organizations that have $101-200$ employees (comprising 6.9\%), then organizations with ' $21-50$ employees' are presented by 16 representatives (7.4\%), and finally $8.3 \%$ of employees belong to '201 - 500 employees' organizations. It is worthwhile noting that only 3 respondents skipped this question and 216 answered it.

It is important to note here that having about $54 \%$ of the respondents coming from large organizations can give the results of the questionnaire more credits as these organizations would most likely have well-defined software development processes, and for being certified such as CMMI. 


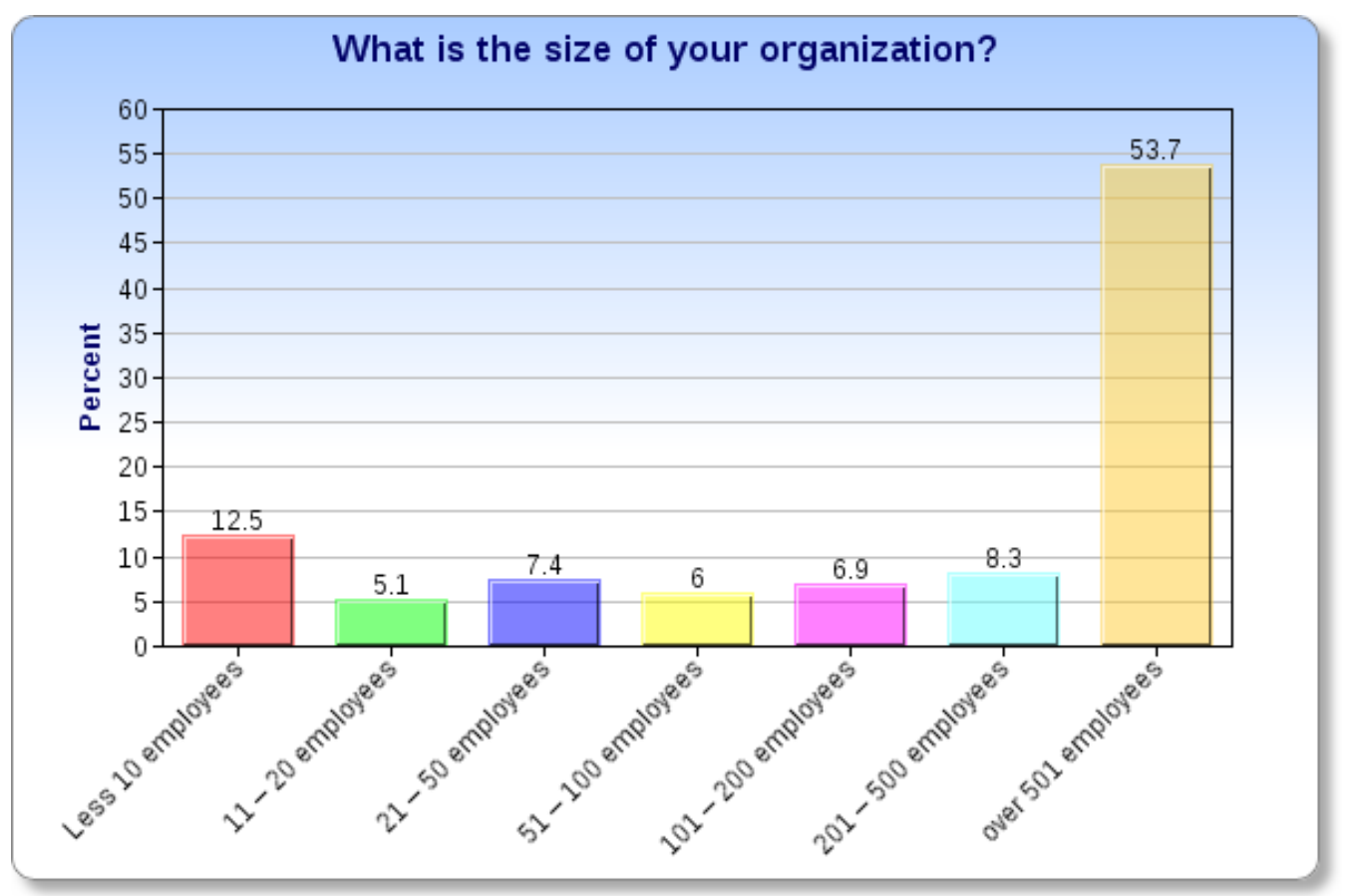

Figure 6: Size of organizations

\section{Average duration of projects}

The respondents are asked to choose the average duration of the projects they were involved in (single choice), (See question 13 in Appendix A).

As shown in Figure 7 around quarter of the respondents (24.6\%) are involved in projects that last between one to two years. And $23.7 \%$ of responses belonged to people who worked in projects between four months and a half year. The third category $(20.8 \%)$ is comprised of respondents who work in projects from half a year to one complete year. Moreover, long duration projects i.e. over three years have obtained $15 \%$ of the responses, and projects that live between two to three years obtained only $9.2 \%$ of the responses. Short duration projects i.e. less than three months received the lowest number of responses i.e. 14 responses out of $219(6.8 \%)$. Note that this question was not answered by 12 people.

It is clear from Figure 7 that the differences among project durations between $4-6$ months, $7-12$ months, and $12-24$ months, are not large ones, and the values are closed to each other. In total, around half of the respondents $(\sim 45 \%)$ are involved in projects that lasted for less than a year, and quarter of the people are involved in projects between one to two years. Additionally, the last quarter goes to projects that take more than two years of development. 


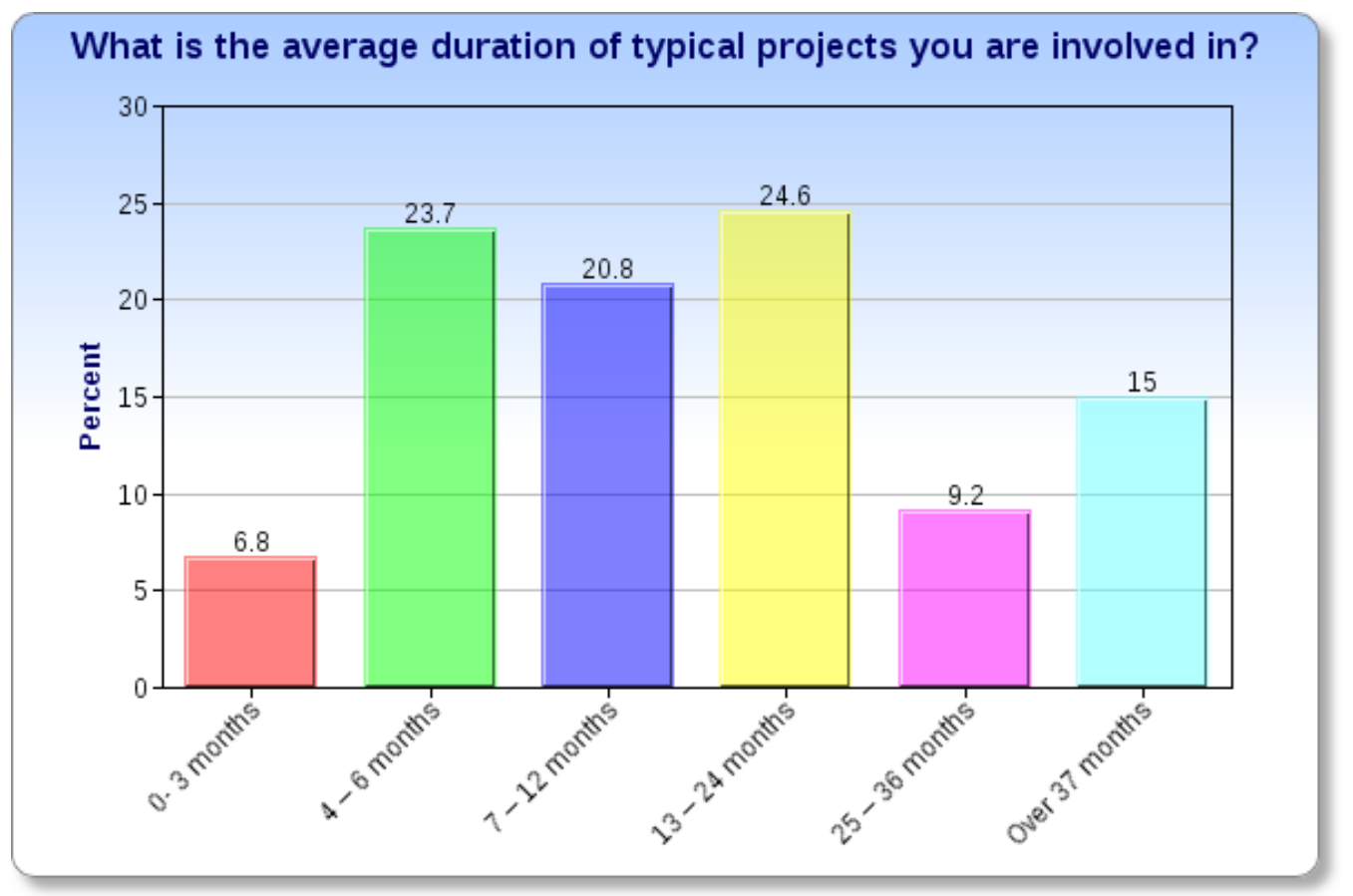

Figure 7: Average duration of projects

\section{Development methodologies and processes}

It is possible that an experienced software developer or manager has used more than one development methodology throughout his/her career. Therefore, the respondents are allowed to choose more than one development methodology as well as to specify others in case the methodology is not listed (multiple choice), (See question 14 in Appendix A).

It is worthwhile noting that the results of this question are computed based on the number of responses received for every category. Since the respondent is allowed to choose more than one category, the computation is done over the number of responses and not over the number of respondents (as in person).

As depicted in Figure 8, agile software development is topping the list of software methodologies with approximately quarter of the respondents using it $(23.6 \%)$. In the second place come the incremental and evolutionary methodology with about $18.8 \%$ of responses. Surprisingly, waterfall methodology is still common and widely used. It came in the third place with $17.7 \%$. Then, the other methodologies receive $9 \%$ and less, and they are as follows: prototyping (9\%), rational unified process $(7.6 \%)$, components-based software development (5.7\%), ad hoc (no method) (5\%), rapid application development (RAD) (4.3\%), spiral development (3.8\%) and finally the formal methods $(2.6 \%)$. Furthermore, some respondents have written names of development methodologies that have been moved to their corresponding category such as scrum or XP which are considered in this case agile. In total, 6 responses were moved to a predefined category, or deleted because the respondent commented on his/her answer. The "others" category (2.4\%) was analyzed. Most of the respondents reported that they mix several methodologies, or they have their own tailored methodology. Finally, 6 people did not answer the question. 


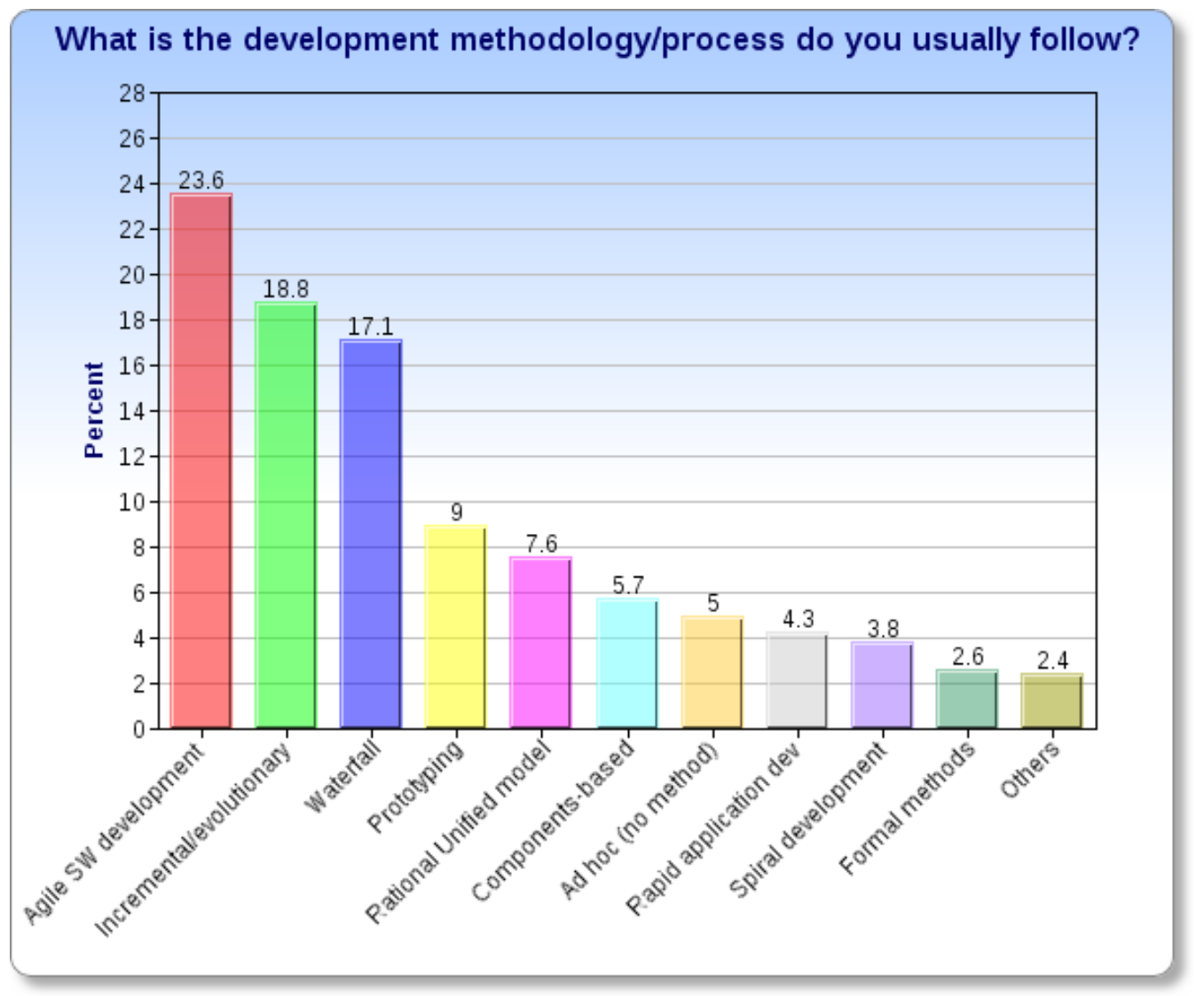

Figure 8: Development methodologies and processes

\section{Types of requirements engineering}

The respondents are asked to choose the types of requirements engineering they have been involved in (multiple choice), (See question 15 in Appendix A). Nevertheless, the person can include "others" by specifying other types of RE. Four types of RE have been listed i.e. bespoke requirements, market-driven requirements, outsourcing, and open source.

The results of this question are computed based on the number of responses received for every category. Since the respondent is allowed to choose more than one category, the computation is done over the number of responses and not over the number of respondents (as in person).

Figure 9 shows that $44.2 \%$ of respondents have chosen bespoke projects. In other words, the majority of respondents worked in projects that are made for certain customers. In the second place come the market-driven projects answer by around $29.4 \%$ of responses, followed by outsourced projects (19.9\%), and finally the open source projects come with only $5.1 \%$ of responses. There are 9 respondents who specified "others" category. Nevertheless, after looking at their descriptions, 4 of the specified types belong to bespoke projects such as in-house development, railway project, etc. One person answered "none". The other four people's answers are: "Normal flow. Requirement from product owner or developer themselves", "Large SAP Implementation projects in a client organization", "mainly infrastructure", and "builds".

Moreover, only 2 people skipped answering this question, and 217 people answered it based on their experience. 


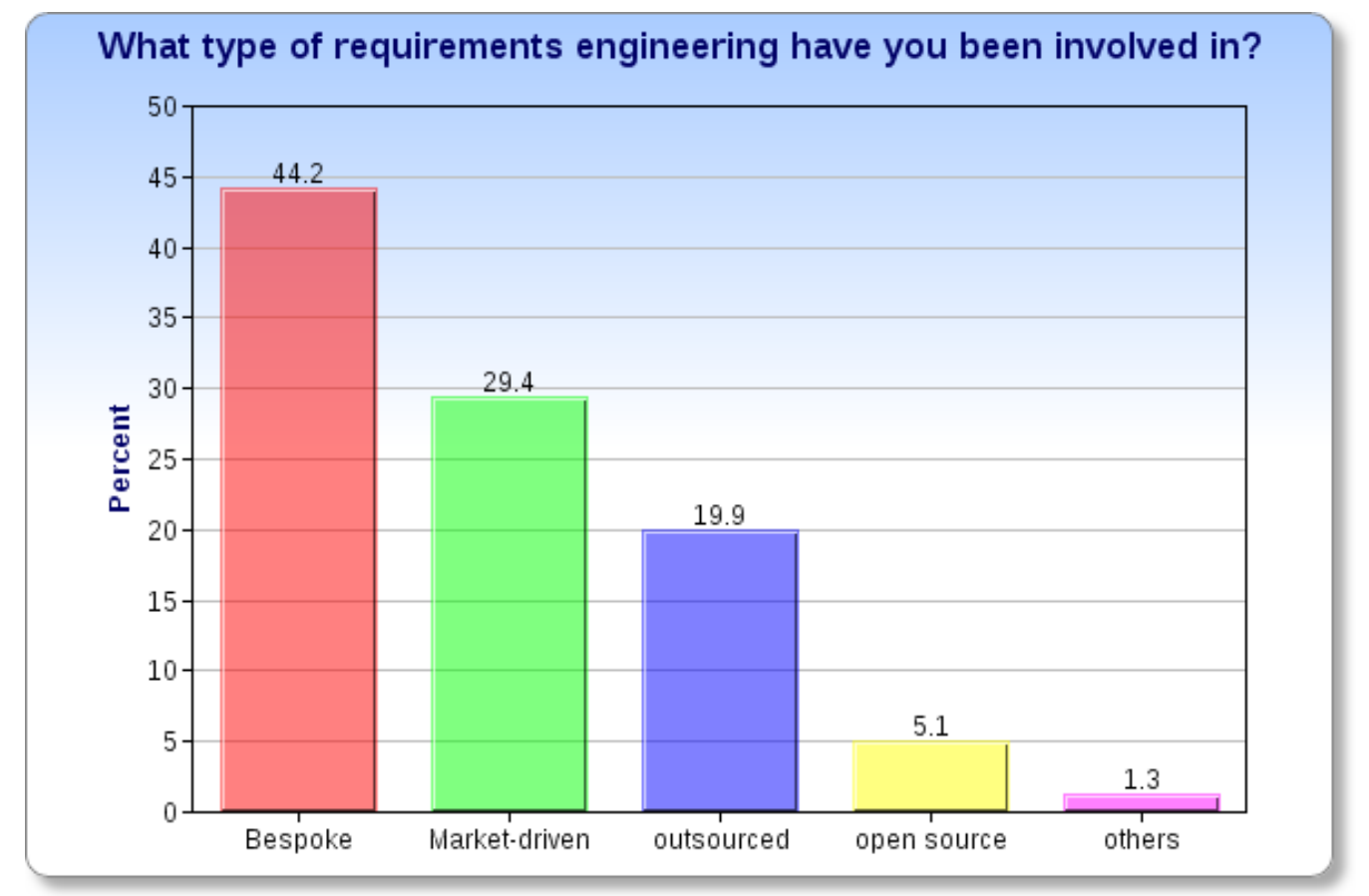

Figure 9: Requirements engineering projects types

\subsection{Statistical methods}

This section briefly describes the statistical methods that are used to analyze the results of the questionnaire (both sections demographic and OSRs).

\subsubsection{Likert Scale}

Likert scale is one of the most commonly used scales in questionnaires. It measures the agreement or disagreement of the respondents with a statement on a scale of five points (sometimes seven or nine points). The middle point of the scale usually represents a neutral opinion about the statement. The scale is usually ordered from the agreement $(1=$ strongly agree, $2=$ agree $)$, then the neutrality $(3=$ Neither, neutral $)$, and finally the disagreement ( $4=$ disagree, $5=$ strongly disagree) [35]. Likert scale data can be treated as interval scale or ordinal scale. However, in this master thesis, Likert scale is treated as an ordinal scale.

Descriptive analysis (mainly median and mean) are used to analyze and interpret the results of Likert scale data. Statisticians agree that the best measure to analyze ordinal scale is through the median ("the category of the variable that represents the center, or the midpoint of the data" [35]). However, statisticians argue about the suitability of using the median with Likert scale to draw proper conclusions of the data. Many statisticians believe that using the arithmetic mean (average) is more suitable in the case of analyzing the results of series of questions that use Likert scale [35]. The use of mean (average) allow the researchers to draw better conclusions, and point out important information about the obtained data. As a result, in this master thesis, the analysis of questions in the questionnaire that use Likert scale will be based on the mean.

\subsubsection{Chi-square test of significance}

Chi-square test of significance is used to find statistical significance (relationship) between two categorical variables (usually of nominal scale). Chi-square test "is essentially concerned with the differences between the frequencies that are obtained from the sample survey and those that could be expected to be obtained if there were 
no differences among the categories of the variables" [35]. In other words, the goal of chi-square test is to find if there is a statistical difference between observed values and expected values. As well, to find whether this difference is due to sampling errors or not. For more information about the meanings of observed frequencies and expected frequencies, the reader can refer to [35].

In general, there are two assumptions associated with chi-square test. The first assumption is called the null hypothesis $\mathrm{HO}, \mathrm{HO}$ assumes no significant relationship among the categories of the studied variables. While, the alternatives hypothesis $H 1$, assumes a significant relationship among the categories of the studied variables.

Chi-square $\left(\mathrm{X}^{2}\right)$ is computed using the following equation:

$$
X^{2}=\sum_{i=1}^{m} \frac{\left(O_{i}-E_{i}\right)^{2}}{E_{i}}
$$

Where:

$\mathrm{O}_{\mathrm{i}}=$ the observed frequency of each cell

$\mathrm{E}_{\mathrm{i}}=$ the expected frequency in each cell

The critical chi-square value determines whether the researcher can accept or reject the null hypothesis $\mathrm{HO}$ or the alternative hypothesis $H 1$. Critical chi-square value needs a couple of information. First, the level of confidence that the research accepts the results of chi-square (95\% or 0.05$)$. Second, the degrees of freedom which is "the number of cells that are free to vary" [35]. The degree of freedom is computed using this formula: $d f=(c-1)(r-1)$, where $d f$ is the degree of freedom, $c$ is the number of categories of the column variable, $r$ is the number of categories of the row variable.

After obtaining the chi-square value and the degrees of freedom, the researcher can find the critical values of chi-square from the table of critical values of chi-square distributions (like the one provided in page 186 in [35]). After knowing the critical value of chi-square, the researcher is able to reject the hypotheses if the computed chisquare value is less than the critical chi-square value. Otherwise, the researcher could accept the hypotheses.

Moreover, the validity of chi-square test of significance results depends on other considerations that the researcher should pay attention to when conducting and interpreting the results. According to the Online Help of SPSS version 17, the considerations are: "The expected frequencies for each category should be at least 1", and "no more than $20 \%$ of the categories should have expected frequencies of less than 5 " [36]. If these conditions are not met, the chi-square results might be invalid.

\subsubsection{Cramer's V}

Chi-square test of significance (section 2.3.2) determines the existence of a relationship between two variables. On the other hand, Cramer's V measures the strength of the relationship between the two variables (nominal scale). The association ranges from 0 to 1 . In other words, $\mathrm{V}=0$ means "no association", and $\mathrm{V}=1$ means "perfect association" [35]. V or Cramer's V is calculated using the following formula:

$$
\text { Cramer's } V=\sqrt{\frac{\mathrm{X}^{2}}{N \min (r-1 ; c-1)}}
$$

where:

$\mathrm{X}^{2}=$ calculated chi-square

$\mathrm{N}=$ sample size

$\min (\mathrm{r}-1 ; \mathrm{c}-1)=$ minimum number of rows or columns

When the value of Cramer's V is between 0 and 1, the value could be interpreted according to the measures of the association (Table 3). 
Table 3: Interpretation of calculated Cramer's V, adapted from [35]

\begin{tabular}{|l|l|}
\hline Measure & Interpretation \\
\hline 0.00 and under 0.10 & Negligible association \\
\hline 0.10 and under 0.20 & Weak association \\
\hline 0.20 and under 0.40 & Moderate association \\
\hline 0.40 and under 0.60 & Relatively strong association \\
\hline 0.60 and under 0.80 & Strong association \\
\hline 0.80 to 1.00 & Very strong association \\
\hline
\end{tabular}




\section{Obsolete Software Requirements Definition and EXISTENCE IN LiTERATURE AND INDUSTRY}

This section defines obsolete software requirements, discusses the meaning of obsolete and its synonyms, and explores the existence of obsolete software requirements in both research literature and industry. This section corresponds to research question number 2 in Table 1 concerning the definition of OSRs, and to research question number 7 concerning finding evidence about OSRs in the industry.

\subsection{Meaning and Synonyms}

The meanings of the word obsolete might be confusing to some readers. Therefore, this sub-section is devoted to its meanings in English, and to identify its synonyms.

"Oxford Dictionaries Online" website defines obsolete as "no longer produced or used; out of date" [37]. As well, "Cambridge Dictionaries Online" website defines obsolete as "not in use any more, having been replaced by something newer and better or more fashionable" [38]. It is evident from the language perspective that obsolete is something that is no longer in use or in production or has been replaced by a newer thing. The definitions give the first clue to the definition of OSRs (see section 3.2).

The synonyms of obsolete according to the Free Online Thesaurus of Synonyms and Antonyms are "outdated", and "out-of-date" [39]. However, outdated and out-ofdate are exchangeable in English. "Deprecated" is another term that is considered of relevance to obsolete which means "to say that you think something is of little value or importance" [40]. In this thesis, the word obsolete will be used to mean out-of-date, outdated, and/or deprecated requirements.

\subsection{Definition of obsolete software requirements}

The following definition of an obsolete software requirement emerged as a result of this thesis:

An obsolete software requirement is a software requirement (implemented or not) that is no longer required for the current release or future releases, and it has no value (business goals) for the potential customers or users of a software product for various reasons e.g. (technological advancements).

The following paragraphs show the readers how this definition was derived and developed.

The questionnaire contains a question (see question 1 in Appendix A) that asked how the respondents define an obsolete software requirement (see Figure 10). This question can be answered by predefined definitions provided by the author. Moreover, the respondent has the ability to choose more than one definition (multiple choice) and/or add his/her own answer. Thus, the results presented below is computed based on the number of unique responses received for every category, and divided on the total number of responses of all categories. It is not computed based on the number of responses divided by the number of respondents (as in person).

On the other hand, the choices were either inspired from the few definitions found in the literature (see section 3.3) or suggested by the author.

Figure 10 shows that the majority of respondents $29.8 \%$ believe that an obsolete software requirement is no longer required for the current release for various reasons. Furthermore, $21 \%$ of the respondents believe that an OSR has no value for the potential users in the current release. $16.3 \%$ of the respondents defined it as "was never used or implemented in the product". Moreover, $14.5 \%$ of respondents believe that it is duplicated/redundant in the current release, and finally $10.7 \%$ of them think that an OSR is rejected/refused to be included in the current release for various 
reasons. So far, it is important to note that many respondents selected more than one choice at the same time.

In addition to, 33 responses $(7.7 \%)$ were written down in the "others" category. After analyzing them, 8 people suggested that an OSR is not confined to the current release, but it also goes to the future releases. Other respondents stressed that an OSR loses its business goals or values. Further, a couple of people distinguished between implemented and non-implemented requirements. As a result, these points have been considered in the original definition provided earlier. Moreover, 7 responses were identified as irrelevant or do not contribute to the definition of OSRs. Note that only 1 person skipped answering this question.

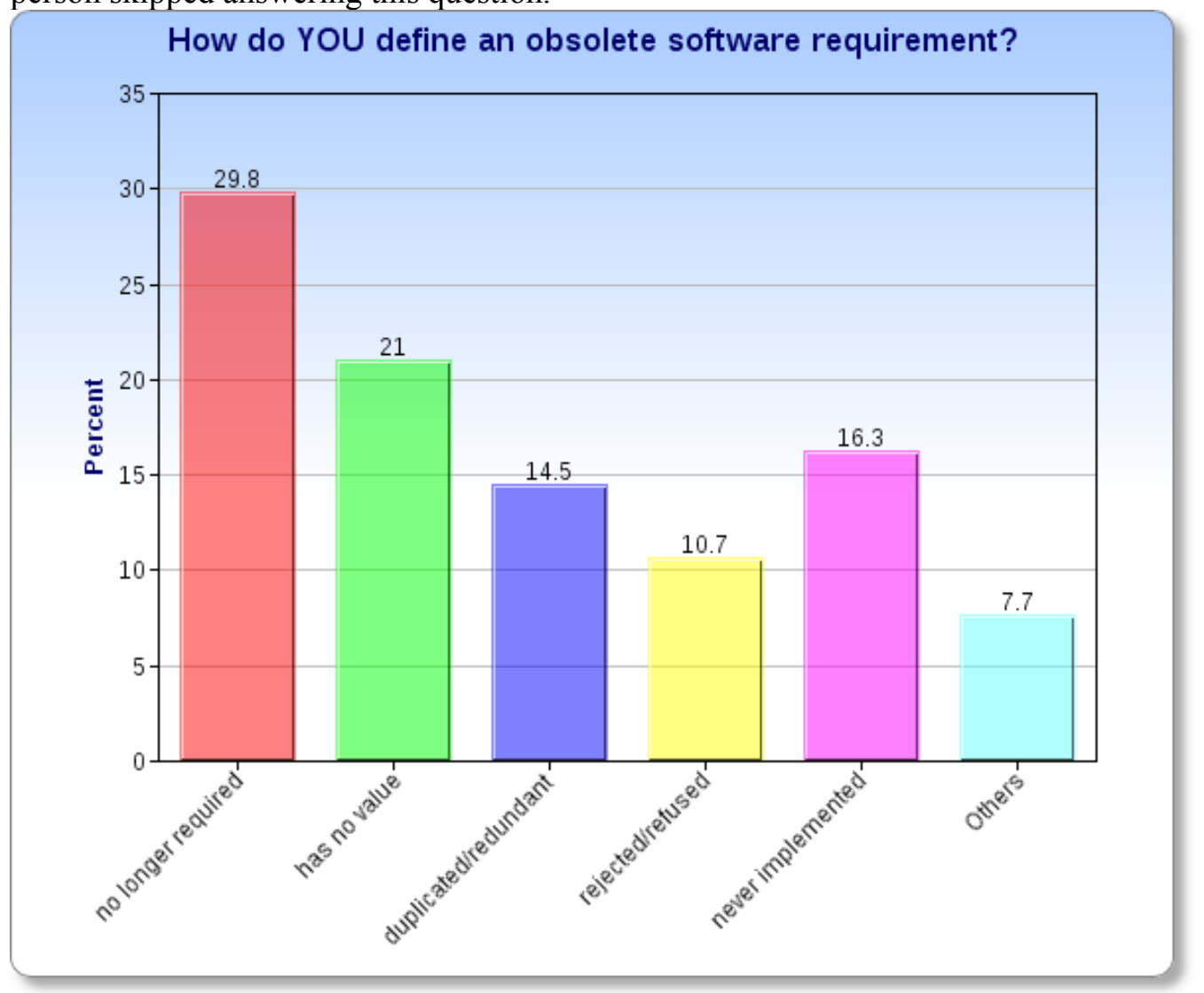

Figure 10: definition of obsolete software requirements

The author investigates the results of the questionnaire further in order to find if there is any relationship between the definition of OSRs on one side, and the respondents roles, the size of organizations, and the development methodologies, respectively, on the other side. This is done using chi-square test of significance (section 2.3.2).

To remind the reader, section 2.2.3 reports the results of the demographic parts of the questionnaire. As shown in Figure 4, the respondents of the questionnaire work in different roles in their respective organizations. Approximately, quarter of the respondents $(24.9 \%)$ work as requirements specialists, and researchers/academicians comprise $11.5 \%$ of the respondents (see Figure 4 in section 2.2.3 for more information).

The null hypothesis (HO) assumes that there is no significant relationship between the respondents roles and the definition of OSRs. While, the alternative hypothesis (H1) predicts a significant relationship between the two variables. 
Chi-square test requires that at most $20 \%$ of the cells have predicted value less than 5. But, there are some roles like 'system integrator' and 'software maintainer' that received 3 and 2 responses, respectively. These two categories were merged with the 'others' category in order to reduce the number of cells that received less than 5.

After running chi-square significance test, the results revealed that the null hypothesis (HO) is not rejected, therefore, there is no significant relationship between the respondents' roles and the definition of OSRs $\left(\mathrm{X}^{2}=44.228, \mathrm{df}=48, \mathrm{p}=0.628>\right.$ 0.05 ). Additionally, there are more than $20 \%$ of the cells have expected count less than 5 , this violates one of the chi-square test conditions that at most $20 \%$ of the cells have expected values less than 5 .

Furthermore, the majority of respondents $(53.7 \%)$ work for large organizations (over 501 employees), followed by small organizations (less than 10 employees) with only $12.5 \%$ of the respondents (see Figure 6 for the rest of the responses). Chi-square significance test is used to find a significant relationship between the size of the organization and the definition of OSRs. The null hypothesis (HO) says that there is no significant relationship between the organizations size and the definition of OSRs. Besides, the alternative hypothesis (H1) assumes a significant relationship between the two variables.

The chi-square test results suggest that the null hypothesis (HO) cannot be rejected $\left(\mathrm{X}^{2}=32.897, \mathrm{df}=36, \mathrm{p}=0.617>0.05\right)$. In addition, in this test, one of chi-square test conditions was violated i.e. more than $20 \%$ of cells have expected cell counts less than 5. Thus, chi-square results may be invalid. As a result, there is no significant relationship between the definition of OSRs and the size of the organizations.

Looking at the data from another aspect, the majority of the respondents practice agile development methods $(23.6 \%)$, whereas waterfall development methodologies are practiced by $17.1 \%$ (see Figure 8 for more results). It is known that agile methods welcome changes to the project during any time whereas waterfall methods address changes in a rigid manner, and mainly during the requirements phase. Therefore, the author searches for an association between the development methodologies practiced by the respondents and the definition of OSRs. He hypothesizes that there is no significant relationship between the definition of OSRs and the developments methodologies (null hypothesis (HO)).

In order to reduce the number of cells that could have a count less than one, the category "others" was excluded from the development methodologies variable.

Chi-square significance tests results show that the null hypothesis $(\mathrm{HO})$ is rejected $\left(\mathrm{X}^{2}=94.03, \mathrm{df}=60, \mathrm{p}=0.003<0.05\right)$. Although there seems to be a significant relationship between the definition of OSRs and the development methodologies, there are two violations of chi-square test conditions that make the results invalid. The violations are: there are more than $20 \%$ of cells have expected cell counts less than 5 , and some cells have values of less than one. These reasons invalidate the results of the chi-square test. As a result, the null hypothesis (HO) is accepted. Thus, there is no significant association between the two variables.

However, Table 4 shows the contingency table between the developments methodologies and the definition of OSRs. In case the reader believes that the null hypothesis $\mathrm{HO}$ is rejected and there is a relationship between the two variables, even though there are violations of chi-square test conditions. The reader can refer to Table 4 and study the relationships.

To conclude, there is no statistical significant relationship between the definition of OSRs provided at the beginning of this section and the respondents roles, the size of organizations, and the development methodologies. Thus, the definition can be generalized. 
Table 4: contingency table between development methodologies and OSRs definition.

\begin{tabular}{|l|l|l|l|l|l|l|}
\hline \multicolumn{2}{|c}{$\begin{array}{l}\text { Not } \\
\text { required }\end{array}$} & \multicolumn{2}{c|}{ No value duplicat } & rejected & \multicolumn{1}{l|}{$\begin{array}{l}\text { Never } \\
\text { impleme } \\
\text { nted }\end{array}$} & others \\
\hline Ad hoc (no method) & $9.10 \%$ & $10.50 \%$ & $6.80 \%$ & $9.80 \%$ & $7.60 \%$ & $11.10 \%$ \\
\hline Waterfall & $37.20 \%$ & $32.60 \%$ & $32.20 \%$ & $41.50 \%$ & $45.50 \%$ & $40.70 \%$ \\
\hline Incremental/evoltu. & $42.10 \%$ & $37.20 \%$ & $42.40 \%$ & $39.00 \%$ & $33.30 \%$ & $48.10 \%$ \\
\hline Spiral development & $6.60 \%$ & $5.80 \%$ & $8.50 \%$ & $14.60 \%$ & $10.60 \%$ & $14.80 \%$ \\
\hline Agile development & $43.00 \%$ & $48.80 \%$ & $47.50 \%$ & $48.80 \%$ & $53.00 \%$ & $74.10 \%$ \\
\hline Rapid App. dev. & $7.40 \%$ & $10.50 \%$ & $8.50 \%$ & $7.30 \%$ & $7.60 \%$ & $22.20 \%$ \\
\hline Prototyping & $19.00 \%$ & $15.10 \%$ & $13.60 \%$ & $19.50 \%$ & $21.20 \%$ & $37.00 \%$ \\
\hline Rational Unified & $9.90 \%$ & $11.60 \%$ & $10.20 \%$ & $7.30 \%$ & $16.70 \%$ & $33.30 \%$ \\
\hline Components-based & $8.30 \%$ & $11.60 \%$ & $16.90 \%$ & $9.80 \%$ & $9.10 \%$ & $22.20 \%$ \\
\hline Formal methods & $6.60 \%$ & $4.70 \%$ & $8.50 \%$ & $2.40 \%$ & $0.00 \%$ & $7.40 \%$ \\
\hline
\end{tabular}

\subsection{Obsolete software requirements in the literature}

The IEEE Software Requirements Specification (SRS) document which is used to document requirements specification does not mention OSRs in its parts. The "IEEE Recommended Practice for Software Requirements Specification" [41] which describes the best practices for writing SRS documents does not talk about OSRs either. Additionally, the latest version of the Capability Maturity Model Integration (CMMI) for development version 1.3 [42] does not address OSRs. The previously mentioned examples indicate that the phenomenon of OSRs is not recognized by experts who issue standards and provide guidelines for practitioners and academicians. Even though, few researchers and authors recognize the presence of OSRs without studying the phenomenon (next paragraphs).

On the other hand, the phenomenon of OSRs seems to be absent in the literature that is related to software engineering or to requirements engineering (section 3.3.1). Few articles and books mention the term obsolete requirements or obsolete features, or that some requirements become obsolete over the course of time. For instance, according to Loesch and E. Ploedereder "these features are likely to be features that have become obsolete" [27]. In addition to, the Software Engineering Body of Knowledge (SWEBOK) recognizes that some volatile requirements "may be rendered obsolete" [43]. Murphy and Rooney agree with SWEBOK. They consider that the "requirements have a shelf life: the longer the time between gathering and implementation, the greater the risk impact of change. Change makes requirements obsolete" [14]. Moreover, other authors recognize that some requirements will become obsolete and they are supposed to be marked as such in the requirements database, or be included in the documentation for future reference [18], [19].

Nurmuliani, Zowghi, and Powell conducted a series of studies on requirements volatility. They define the term "obsolete functionality" as a "functionality that no longer required for the current release or has no value for the potential users" [20]. Finally, in their book, Leffingwell and Widrig define a "dead feature" as "one that is still in the system but whose reason to exist has disappeared or at least is no longer clear" [44].

In addition to, a recent report from the Institute for Government, UK entitled "System Error - Fixing the flaws in government IT" has recognized obsolete 
requirements as one of the symptoms of failure of IT projects for governments. The report ascribes OSRs mainly to the "rapid pace of change in technology" [45]. Moreover, the report does not provide any definition of what OSRs are.

\subsubsection{The results of the systematic literature review}

In order to find evidence about OSRs in the literature, the SLR was conducted (section 2.1), and its results are reported below. In total, only 3 partially relevant articles were found during the SLR (see Figure 2). The author decided that they are partially relevant to OSRs because the articles mention the notion of OSRs, or talk about it in general. Nonetheless, none of the articles is dedicated to OSRs.

The first article entitled "Language Architecture - Obsolescence and Deletions" [28], describes how a programming language develops (i.e. Fortran), and how some features become obsolete and unnecessary over the course of time. Henceforth, these features have to be deleted from the language. The article provides some evidence that the notion of feature (requirement) obsolescence exists in reality. On the other hand, the author describes a number of obsolete features of the language that were deleted from version to version over the course of time. It also provides the rationale behind the deletions. Nevertheless, the article does not include any reference and it is more of a narrative paper that describes how Fortran Community decides on deleting or leaving features in the language.

The second article is entitled "Restructuring Variability in Software Product Lines Using Concept Analysis of Product Configurations" [27]. As this title implies, the article talks about variability in SPL. What is interesting in this article to this master thesis is a features classification usage provided in the article. Variable features are divided into 4 categories based on their usages:

1) always used

2) never used

3) only used mutually exclusively

4) only used in pairs.

The differences amongst the 4 categories are beyond the scope of the thesis. Yet, the second category (i.e. never used) is relevant to the thesis. It says that the never used features "are likely to be features that have become obsolete. As these variable features are obviously not needed anymore, they should be removed from the set of offered features" [27]. The reason why these variable features are never used is that they have never been used or implemented in any product configurations and there exist other alternatives that are preferred in the product configurations. This forms other evidence that "never used" features become obsolete.

The third article is entitled "Updating Requirements from Tests During Maintenance and Evolution" [26]. In his article, Charrada introduces a new concept to update software requirements as the documentation is usually outdated, and in many cases it contains many obsolete requirements and specifications. The idea is to use "high-order tests" (acceptance, function, and system tests) "as a link between the requirements and implementation" [26]. Using high-order tests was due to that these test themselves "deal with the visible behavior of the system" [26], and are usually directly derived from the requirements. High-order tests usually check that the built system meets the specified requirements in the documentation. This method helps updating the requirements. As a result, the number of OSRs in the documentation is reduced.

\subsubsection{Obsolete software artefacts}

Obsolescence of software or software artefacts (e.g. Commercial-off-the-Shelf COTS) is different from software requirements obsolescence. Software obsolescence has been discussed in the literature mainly in conjunction with hardware and 
electronics obsolescence in the military, avionics and electronics industries, or, in other words, Diminishing Manufacturing Sources and Material Shortages (DMSMS).

Herald et al. define obsolescence as "when a part (hardware, software, constraint) is no longer able to perform its required function such as: availability for purchase or ability to be repaired affordably" [46]. However, Merola's definition says that "Software applications become obsolete when they are retired from use and taken off the market due to technology advancements, decrease in product popularity, or other market factors. Vendors may no longer sell or support licenses for older versions of the application" [21].

To sum, it is evident here that software obsolescence is another topic and different from OSRs even though the former might have effects on the latter.

\subsection{Seriousness of obsolete software requirements in the industry}

The author assumed that the phenomenon of OSRs exists in reality (i.e. industry). Thus, one of the questions in the questionnaire was to find out how serious this phenomenon is perceived by the respondents (See question 2 in Appendix A).

As shown in Figure 11, 44.7\% of respondents consider OSRs somehow serious, $39.6 \%$ believe it is a serious matter, and only $6 \%$ perceive it as very serious. On the other hand, only 21 respondents out of $219(9.7 \%)$ deem OSRs as a trivial matter. Moreover, the mean (average) value of the responses is 2.42 , the mean indicates that OSRs is between 'somehow serious' and 'serious'. Note that only 2 people skipped answering this question.

In addition to the results of the questionnaire, a recent report from the Institute for Government, UK entitled "System Error - Fixing the flaws in government IT" has recognized obsolete requirements as one of the symptoms of failure of IT projects for governments. The report ascribes OSRs mainly to the "rapid pace of change in technology" [45].

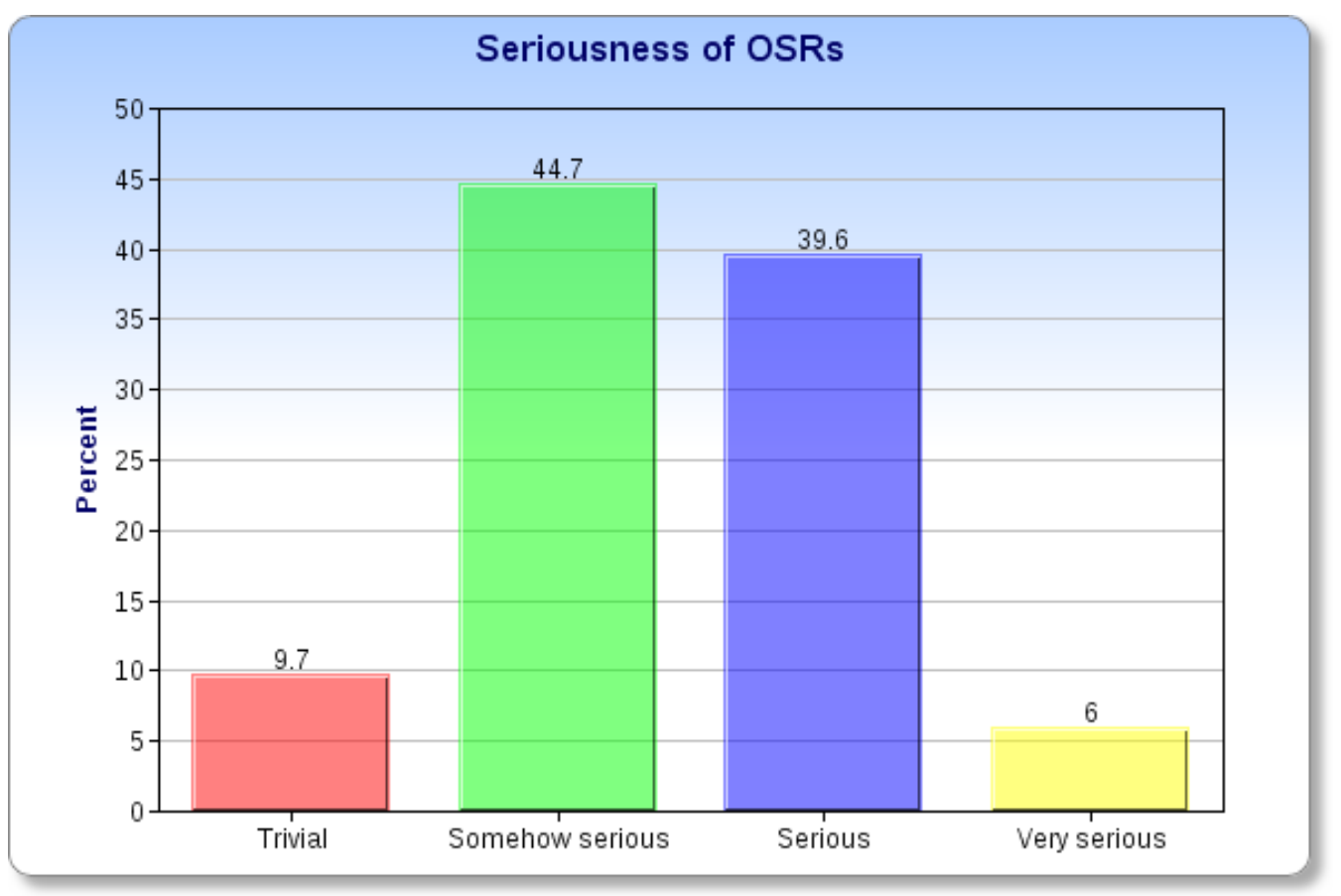

Figure 11: Seriousness of obsolete software requirements

The results shown in Figure 11 can statistically be analyzed further. There might exist a significant relationship between the seriousness of OSRs (variable 1) on one 
side, and the respondents roles (variable 2), the size of organizations (variable 2), and the development methodologies (variable 2), respectively, on the other side.

Figure 4 in section 2.2.3 shows that requirements specialists comprise quarter of the respondents of the questionnaire. This is followed by the researchers/academicians who make $11.5 \%$ of the total respondents (see Figure 4 in section 2.2.3 for more results). There could be a significant association between the respondents' roles and the seriousness of OSRs (alternative hypothesis H1). In order to find this association, chisquare significance test was run on the data using SPSS. Chi-square significance tests revealed that $H 1$ is rejected. So, the null hypothesis $(H O)$ is accepted i.e. there is no significant association between the respondents' roles and the seriousness of OSRs $\left(\mathrm{X}^{2}\right.$ $=20.363, \mathrm{df}=24, \mathrm{p}=0.68>0.05$ as shown in Table 5). Additionally, the note beneath Table 5 says that $52.8 \%$ of the cells have expected count less than 5 , this violates one of the chi-square test conditions that at most $20 \%$ of the cells have expected values less than 5. It is important to mention here that the roles 'system integrator' and 'software maintainer' that received 3 and 2 responses, respectively, were merged with the 'others' category. This merge was done so as to reduce the number of cells that received less than 5 . Yet, there is no significant relationship.

Table 5: Chi-Square test for seriousness of OSRs and respondents' roles

\begin{tabular}{|l|l|l|l|}
\hline & Value & df & Asymp. Sig. (2-sided) \\
\hline Pearson Chi-Square & $20.363^{\mathrm{a}}$ & 24 & 0.68 \\
\hline Likelihood Ratio & 23.32 & 24 & 0.50 \\
\hline Linear-by-Linear Association & 0.72 & 1 & 0.40 \\
\hline N of Valid Cases & 215 & & \\
\hline
\end{tabular}

a. 19 cells $(52.8 \%)$ have expected count less than 5 . The minimum expected count is .73 .

Furthermore, section 2.2.3 shows that majority of respondents (53.7\%) belong to organizations that have over 501 employees, followed by small organizations that employee less than 10 employees ( $12.5 \%$ only) (the rest of the responses are shown in Figure 6). Using chi-square test of significance, one can find whether there is a significant relationship between the size of the organizations and the seriousness of OSRs (alternative hypothesis H1) or there is no significant relationship between the two variables (null hypothesis HO) .

The results of chi-square significance test obtained using SPSS are shown in Table 6. It is obvious from the note under Table 6 that half of the cells have expected values less than 5 , this is a violation of the chi-square test condition that at most $20 \%$ of the cells have values less than 5 . Moreover, chi-square test result $\left(X^{2}=10.38, \mathrm{df}=18, \mathrm{p}=\right.$ $0.92>0.05$ ) suggests that the null hypothesis $H 0$ cannot be rejected because $\mathrm{p}=0.92$ which is greater than 0.05 . This means that there is no statistical significance between the two variables (seriousness of OSRs and the size of the organizations). As a result, the results shown in Figure 11 can be generalized.

Table 6: Chi-Square test for seriousness of OSRs and organizations' size

\begin{tabular}{|l|l|l|l|}
\hline & Value & $\boldsymbol{d f}$ & Asymp. Sig. (2-sided) \\
\hline Pearson Chi-Square & $10.380^{\mathrm{a}}$ & 18 & 0.92 \\
\hline Likelihood Ratio & 12.42 & 18 & 0.83 \\
\hline Linear-by-Linear Association & 0.50 & 1 & 0.48 \\
\hline N of Valid Cases & 214 & & \\
\hline
\end{tabular}

a. 14 cells $(50.0 \%)$ have expected count less than 5 . The minimum expected count is .67 . 
On the other hand, the majority of respondents practice agile methods $(23.6 \%)$, whereas waterfall development methodologies are practiced by $17.1 \%$. Agile methods address requirements changes at anytime during the course of the software project, and they have the highest rate of creeping requirements monthly (10\%) [5], as opposed to waterfall methods which address requirements changes mainly during the requirements phase in the SPLC. Here, the author is looking for a statistical relationship between the development methodologies followed by the respondents and the seriousness of OSRs. It is worthwhile noting here that the "others" category from the development methodology variable was excluded, so as to reduce the number of cells that have expected values less than 5 .

The null hypothesis $\mathrm{HO}$ says that there is no significant relationship between seriousness of OSRs and the developments methodologies. After running chi-square significance tests on the data, the results show that the null hypothesis is accepted ( $\mathrm{X}^{2}$ $=39.38, \mathrm{df}=30, \mathrm{p}=0.117>0.05$ ). Moreover, there are more than $20 \%$ of cells have expected cell counts less than 5, and some cells have expected values of less than one. These violations invalidate the results of the chi-square test. As a result, the results shown in Figure 11 can be generalized.

To sum, according to the results of chi-square test of significance, there is no statistical significance between the seriousness of the OSRs on one side, and the respondents roles, the size of the organization, the development methodologies, respectively, on the other side.

In conclusion, it is found that a few articles or books mentioned OSRs in different contexts. This indicates their absence in literature even though they exist in reality. Additionally, OSRs is found to be a serious issue in the software engineering context, according to the results of the questionnaire. Moreover, this section defines an obsolete software requirement as a software requirement (implemented or not) that is no longer required for the current release or future releases, and it has no value (business goals) for the potential customers or users of a software product for various reasons. 


\section{Requirements Classifications}

This section develops a software requirements classification based on the concept of requirements volatility. The developed classification helps requirements engineers to find/detect OSRs based on their types. This section corresponds to research question number 3 and 3.1 in Table 1 concerning the relationships between OSRs and software requirements types, and ways of finding OSRs.

Software requirements are not of one type. There are several types of requirements depending on several dimensions like the source requirements, the business needs, and the technology. Indeed, the literature has no one agreed-on requirements classification. Besides, the classifications found in the literature do not necessarily reflect or meet the practices [47].

Aurum and Wohlin [47] summarized a group of classifications of requirements. They are presented in Table 7. It is apparent that the classifications in Table 7 distinguish several types of requirements depending on the characteristics of the requirement itself. For instance, a requirement that belongs to the domain level, or a requirement that is functional or non-functional, or it is elicited from certain stakeholders.

Table 7: Requirements Classification, adapted from [47] except the grey row.

\section{Requirements Classifications}

- Functional requirements - what the system will do.

- Non-functional requirements - constraints on the types of solutions that will meet the functional requirements e.g. accuracy, performance, security and modifiability.

- Goal level requirements - related to business goals.

- Domain level requirements - related to problem area.

- Product level requirements - related to the product.

- Design level requirements - what to build.

- Primary requirements - elicited from stakeholders.

- Derived requirements - derived from primary requirements.

Others classifications, e.g.

- Business requirements versus technical requirements.

- Product requirements versus process requirements - i.e. business needs versus how people will interact with the system.

- Role based requirements, e.g. customer requirements, user requirements, IT requirements, system requirements, and security requirements.

Classification of software requirements knowledge [48]:

- Domain requirements knowledge: requirements obtained from domain experts.

- Customer's requirements knowledge: requirements coming from the customer which is considered the main source of requirements.

- End user's requirements knowledge: requirements originated from the end users of the system.

- Constraints requirements knowledge: requirements acquired from various stakeholders which impose constraints on the system such as the environment, laws, budget etc. 
In addition to [47]'s summary, a recent study about knowledge transfer of software requirements [48] has introduced a new classification of requirements knowledge. The classification of software requirements knowledge is based on the sources of the requirements i.e. sources of knowledge. The requirements have been divided into four types according to this classification. They are presented in Table 7 (highlighted in grey).

The Guide to the Software Engineering Body of Knowledge (SWEBOK) [43] classifies the requirements, but many of its classifications overlap mainly with the classifications in Table 7. Nevertheless, SWEBOK recognizes another type of requirements that is absent from Table 7 . They are volatility/stability requirements [43]. In other words, a stable requirement seldom changes during the duration of the project meanwhile a volatile requirement keeps changing over the course of the project. Indeed, this classification is based on the changing nature of requirements that is an inherent characteristic of them. Also, it is the most suitable one to be used in this master thesis because the "change makes requirements obsolete" [14].

The following paragraphs discuss how the author developed a classification based on the volatile requirements classifications found in the literature.

Harker, Eason, and Dobson [49] categorize software requirements based on their changing nature. Their classification consists of six types of requirements divided into two major groups, namely stable and changing requirements. It was based on the nature and the source of the change in requirements.

Under the stable group, there is only one type of requirements described as enduring. It is connected back to the technical core of the business, and these requirements seldom change over the course of the project whereas the changing requirements group has five types. They are presented with their meanings in Table 8 .

Table 8: Changing Requirements (Volatile) according to [49].

\section{Type of requirement Description}

\begin{tabular}{|l|l|}
\hline Mutable & Originated from environmental turbulence. \\
\hline Emergent & $\begin{array}{l}\text { Acquired from stakeholders engagement in requirements } \\
\text { elicitation. }\end{array}$ \\
\hline Consequential & $\begin{array}{l}\text { Obtained from users after using the system. } \\
\text { Adaptive }\end{array}$ \\
\hline Migration & $\begin{array}{l}\text { Origing from the situation the system is installed in and the } \\
\text { another state. }\end{array}$ \\
\hline
\end{tabular}

Sommerville adapted Harker's classification (in Table 8) and made subtle changes to it. The changing requirements category has been dubbed "volatile requirements", and it has only four types of requirements instead of five. Sommerville retained the first three types of volatile requirements (mutable, emergent and consequential) and added compatibility requirements (requirements which depend on the equipments or the processes) instead of adaptive and migration requirements types [1].

Moreover, McGee and Greer have developed a taxonomy of software requirements change sources [50] based on the knowledge of experienced project managers. Using previous publications, they gathered uncertainties constructs and triggers constructs that would cause a requirement change. Then, they used the card sorting technique with the expert knowledge to classify the sources of requirements changes based on the collected constructs. As a result, the developed taxonomy includes five change domains. Each domain has triggers and uncertainties which will arise/cause the change. Table 9 summarizes the taxonomy of software requirements change source. It 
is important to note that the knowledge experts reported that the lower control of uncertainties is at the top of the list (i.e. market), and the higher control is at the bottom (i.e. solution) [50].

Likewise, in their series of researches on requirements volatility, Nurmuliani et al. [20] have developed a taxonomy for requirements changes. The taxonomy was derived from the change request forms submitted to the company they implemented the study on. After the analysis of the change request forms, they found that $86 \%$ of the requests were related to requirements. Moreover, the devised taxonomy consists of three components that are: change type (addition, modification, deletion), reason for change (e.g. defect fixing, missing requirements), and the origin of the change (e.g. project management considerations, marketing group, technical team discussion).

Table 9: Taxonomy of software requirements change source [50].

\begin{tabular}{|l|l|}
\hline \multicolumn{1}{|c|}{ Change Domain } & $\begin{array}{l}\text { Description } \\
\text { Market }\end{array}$ \\
\hline Customer Organization & $\begin{array}{l}\text { Strategic changes in the customer organization and its } \\
\text { politics, and changes in the customer software and } \\
\text { hardware. }\end{array}$ \\
\hline Project Vision & $\begin{array}{l}\text { Changes concerning business process, cost/schedule, } \\
\text { stakeholder representatives and roles, and emergence of } \\
\text { new opportunities. }\end{array}$ \\
\hline Requirements Specification & $\begin{array}{l}\text { Changes in the specifications of the problem due to } \\
\text { better understanding of the problem by stakeholders, } \\
\text { and resolutions of ambiguous, incomplete, and incorrect } \\
\text { requirements. }\end{array}$ \\
\hline Solution & $\begin{array}{l}\text { Changes related to technical solutions and tools and } \\
\text { technologies used in development. }\end{array}$ \\
\hline
\end{tabular}

After the taxonomy development, the change request forms were analyzed, and sources of change types (addition, modification, deletion) were identified. Modification and deletion are found to cause obsolescence of software requirements [20]. Therefore, the sources and reasons of relevance to OSRs that are summarized in Table 10 are those that led the requirements to be modified or deleted.

It is worthwhile mentioning that Nurmuliani et al. [20] also investigated the causes of requirements changes. Their findings were aligned with Harker's findings [49].

In essence, it is evident from the above-described classifications that there is no agreement on the terminology for classifying requirements. Similarly, there is no one comprehensive requirements classification.

\section{Which requirements classification is more suitable to OSRs?}

The classifications mentioned in Table 7 are of low relevance to OSRs, because they categorize requirements based on the characteristics of the requirement itself, and not on the changing nature of requirements. Since requirements keep changing over the course of the project, classifications such as the ones in Table 8 and Table 9 that recognize the mutability nature of requirements, are more relevant to OSRs. A requirement would mainly become obsolete after changes in the requirement itself. Thus, changes in requirements are the main driver to obsolescence of requirements [14]. As an example, Nurmuliani et al. found that many requirements became obsolete 
after either deleting or modifying them [20]. Put simply, volatile requirements are more eligible to become obsolete than stable requirements are.

Moreover, the classification done by Nurmuliani et al. [20] in Table 10 is only verified by one company. For that reason, it will not be adapted as a classification for this study. However, its results can be helpful to verify the results that will be obtained from this study. Especially Nurmuliani et al. [20] listed several reasons that caused modifications and deletions of requirements which later led them to become obsolete.

Table 10: Sources and reasons of requirements changes that caused deletion or modification of requirements [20].

\begin{tabular}{|l|l|}
\hline Sources of changes & Marketing group \\
\hline Technical team discussions & Project management considerations \\
\hline Design review/feedback & Developer's detailed analysis \\
\hline Feature proposal reviews & Functional specification reviews \\
\hline Customer support discussions & \\
\hline Reasons categories of changes & Obsolete functionality \\
\hline Erroneous requirements & Redundant functionality \\
\hline Design improvement & Clarifying requirements \\
\hline Scope reduction & Resolving conflicts \\
\hline Product strategy &
\end{tabular}

McGee and Greer's taxonomy (Table 9) is influenced by Harker's classification (Table 9) [50]. Moreover, they introduced a mapping between the two classifications: mapping market and customer organization domain sources into mutable requirements, and mapping project vision and requirements specification domains into emergent requirements. Nevertheless, the author found that project vision and requirements specification domains map to both emergent and consequential requirements. Because consequential requirements are obtained after the system is put into use by the end user representatives, and in requirements specification domain the engagement of the user representatives is considered as a trigger of change [50]. Nonetheless, the other types of requirements in both classifications do not map to each other. Table 11 shows the mapping between the two classifications.

Table 11: Mapping between McGee's taxonomy and Harker's classification

\begin{tabular}{|l|l|}
\multicolumn{2}{|c|}{ McGee's Taxonomy } \\
\hline Market & Mutable \\
\hline Customer organization & Mutable \\
\hline Project vision & Emergent and consequential \\
\hline Requirements specification & Emergent and consequential \\
\hline Solutions & - \\
\hline- & Adaptive \\
\hline- & Migration \\
\hline
\end{tabular}

\subsection{Volatile requirements classification}

The hybrid classification (Table 11) will be used in this study to define which types of requirements are more eligible to become obsolete than others. 
The decision to use the hybrid approach was due to its ability to recognize the changeability nature of requirements which in its turn is highly connected to OSRs. As well as, it also covers bigger scope of requirements than any one classification does. However, the names of the types (e.g. mutable, project vision) might be misleading or ambiguous to many people. Therefore, a list of more detailed types of requirements is shown in Table 12. This list is developed by the author of the thesis according to the original descriptions of the categories and types in [49], [50].

Table 12: Detailed lists of requirements types based on the hybrid classification

Mutable $=$ Market and customer organization .

- Requirements related to laws and regulations.

- Requirements related to standards.

- Requirements obtained from market analysis, domain experts and competitors.

- Requirements about the company's organization and policies.

- Hardware related requirements.

Emergent and consequential $=$ project vision and requirements specification.

- Functional requirements originated from customers.

- Functional requirements originated from end users.

- Functional requirements originated from developers.

- Incorrect requirements.

- Ambiguous requirements.

- Inconsistent requirements.

Adaptive.

- Non-functional requirements - portability.

- Adaptability.

- Installability.

Migration.

- Non-functional requirements - portability.

- Replacability.

Solutions.

- Requirements related to third party components e.g. COTS.

- Requirements related to design and architecture.

- Usability requirements.

- Interoperability requirements.

As mentioned earlier, McGee and Greer claim that the requirements in the list are more likely to change as one moves down in the list [50]. This means that as one goes down in the list, the requirements are more likely to become obsolete. This claim is going to be checked in the questionnaire (see below).

The questionnaire contains a question that asks the participants to rate how likely they think the types of requirements listed in Table 12 will become obsolete. The rating is on a scale from 1 to 5 where 1 is 'not likely' while 5 is 'very likely' (see question 3 in Appendix A).

This question was answered by all participants of the questionnaire. Figure 12 shows the original order of types of requirements as shown in Table 12. In Figure 12, the type of requirements are listed on the right side and the average rate is displayed at the end of the horizontal bar. One can clearly see from Figure 12 that the respondents agree to some extent with McGee and Greer's claim [50]. As you go down in the list (left to right in Figure 12), the average rate (mean) of requirements to become obsolete 
gets higher. However, the last 5 types of requirements do not follow the rule, and thus the author believes this is because the list that is developed does not necessarily follow [50]'s claim.

According to the results in Figure 12, incorrect requirements have topped the types with a mean of 3.88. In other words, they are most likely to become obsolete first. This is followed by inconsistent requirements (3.74) and ambiguous requirements (3.72). Note that the differences among the three types are small. It is also important to note that these three categories are common problems in requirements engineering. As well, they tend to change more than any other type, and thus they are more likely to become obsolete.

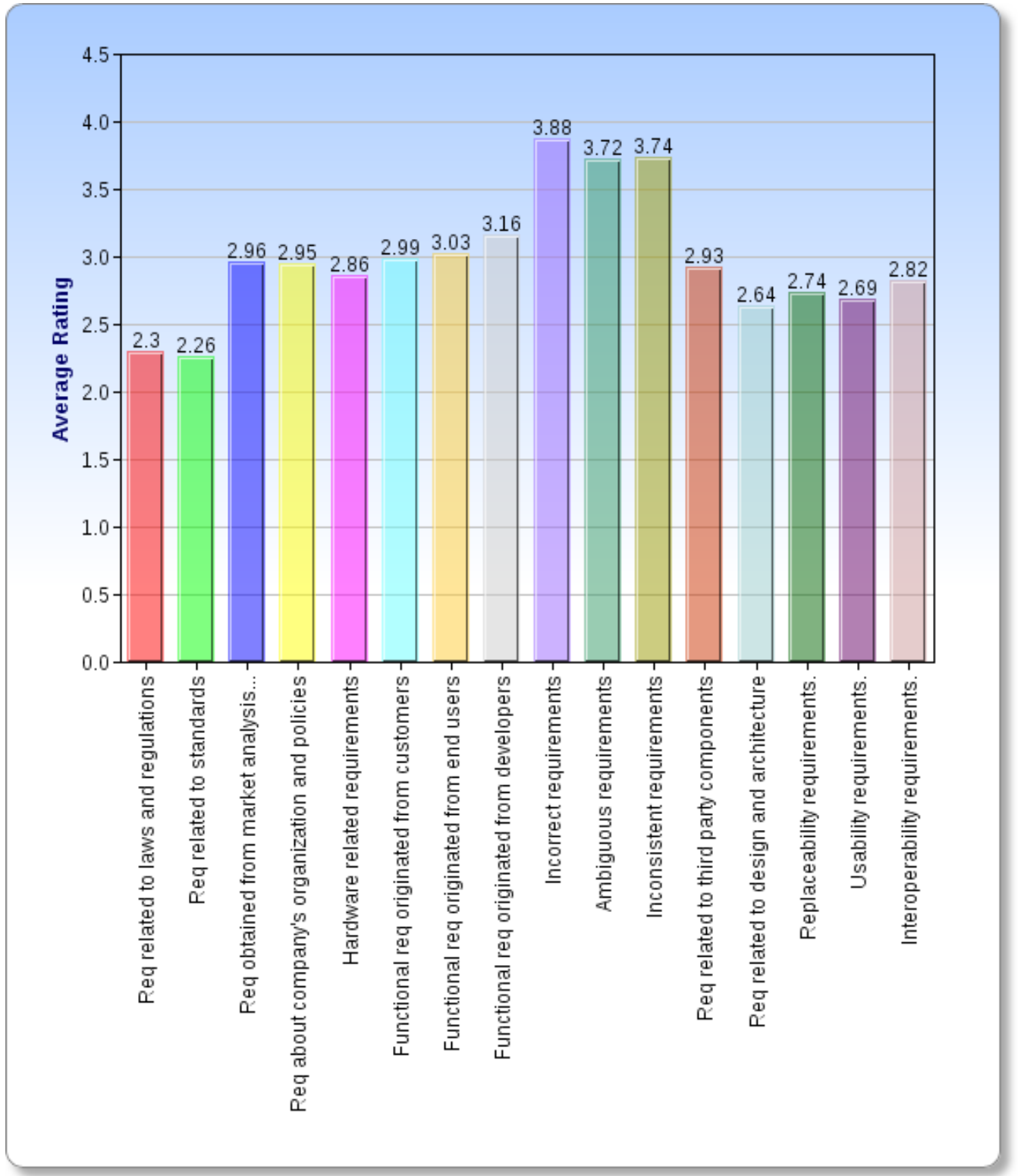

Figure 12: Requirements types and their likeliness to become obsolete (original order)

In Figure 13, the requirements classification is displayed based on the higher average rate (mean) of likeliness to become obsolete. Interestingly, functional requirements coming from experts of domain are less likely to become obsolete than the requirements which come from customers, end users, and developers, respectively. The author believes this order follows the common politics involved in deciding which 
software requirements to include/exclude in the project. Wohlin and Aurum in [9], found that stakeholder priority of requirement and the requirements' issuers are amongst the top five criteria (out of 13) that influence the decision-making process in selecting requirements in software projects or releases. Likewise, Kabbedijk et al. [51] found that change requests received from external customers are more likely to be accepted than internal change requests. Moreover, Wnuk et al. in [52] argue that features implementation is affected by different decisions, these decisions are influenced by different factors such as technological advancements.

Domain experts possess the knowledge and experience of the domain, they are "very familiar with the system requirements, design, and implementation of the software, the use of the software, hardware, and technology etc of this domain" [48]. Thus, requirements coming from domain experts are less likely to change than the requirements coming from customers. The customers are considered the main source of software requirements because they own the problems to be solved by the software. Nevertheless, they do consult domain experts, and the customers' opinion is influenced by domain experts.

Moreover, "end users are the individuals or groups who will use the software to be developed" [48]. Requirements coming from them are important because they are the actual people who use the system. However, they have less influence on the decisionmaking process of inclusion/exclusion of requirements. Finally, developers are the individuals who build the system. They can suggest and propose requirements based on their experience. Yet, their requirements might not be accepted by the customers, and the customers are most likely to prefer their own ideas.

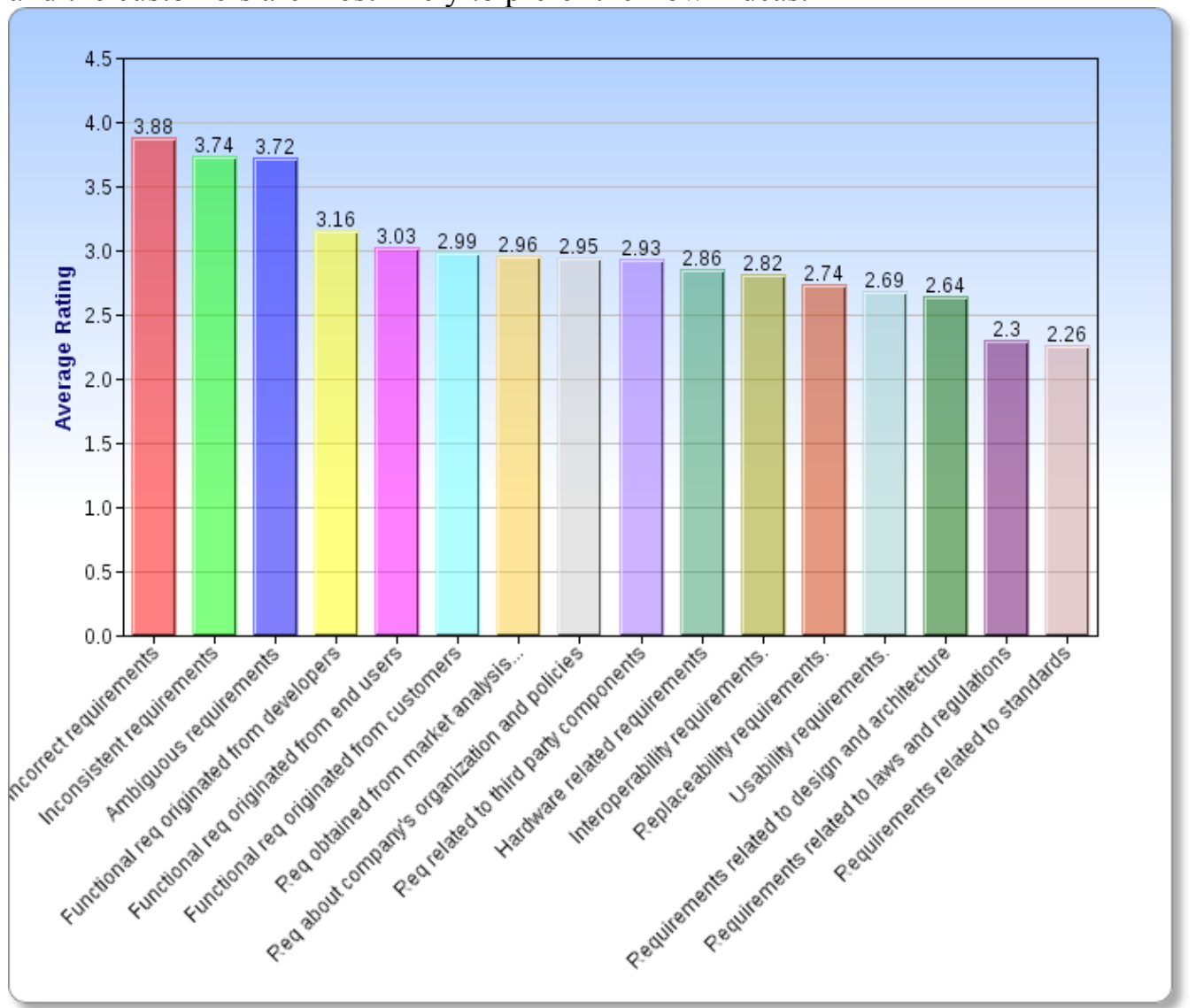

Figure 13: Requirements types and their likeliness to become obsolete (ordered by higher average rate to become obsolete) 
Furthermore, requirements related to standards (2.26) are at the bottom of the list and considered the slowest to become obsolete. Whereas, requirements related to laws (2.3) are more likely to become obsolete than requirements related to standards. Nevertheless, there is a slight difference between the two averages and, in general, requirements related to laws and standards are less likely to become obsolete than any other type as the standards and laws change slowly.

The results of this question (question 3 in Appendix A) are helpful to requirements engineers for early identification of OSRs, or requirements that could become obsolete. Additionally, the results help requirements engineers to specify which requirements they should look at, or read first, when they are reviewing the requirements documentation.

Furthermore, the results could be used as a way to find/detect OSRs as described in section 5.2.3.

In conclusion, this section suggested a classification of software requirements based on their volatility. Also, this section reports that the probability of incorrect requirements, inconsistent requirements, and ambiguous requirements to become obsolete is higher than any other software requirements type. As well, requirements related to standards and laws are the slowest to become obsolete, based on the questionnaire's findings. 


\section{Obsolete Software Requirements Processes}

This section proposes several ways of finding OSRs, as well as ways of handling them after being discovered. It also reports the results of the questionnaire regarding the existence of processes in the industry that take OSRs into account. This section covers research questions number $3.1,4,4.1,4.2$, and 7 in Table 1 concerning processes related to OSRs.

Section 3.4 concludes that the phenomenon of OSRs is considered a serious issue by the majority $(90 \%)$ of the respondents of the questionnaire. Therefore, it is necessary to recognize OSRs in the requirements engineering process of software organizations in order to reduce their negative impact on the project in general.

Furthermore, one of the major goals of this master thesis is to investigate if software organizations recognize OSRs, and if they have processes to deal with them. The questionnaire contains three questions in that regard:

1. A question asking if organizations possess processes that take OSRs into account, (see question 9 in Appendix A).

2. A question about ways of discovering/detecting OSRs, (see question 4 in Appendix A).

3. A question concerning the ways of treating OSRs after being discovered, (see question 5 in Appendix A).

The three questions allow the respondents to choose answers from a predefined set of suggestions, or to provide their own descriptions of their own processes.

This chapter reports the results of the three questions and suggests processes for OSRs. The author proposed the processes such that they are based on the results of the questionnaire or influenced by them.

\subsection{Existence of OSRs process in industry}

Since the literature has no strong recognition of OSRs (section 3.3), it is assumed that there are no explicit processes which handle OSRs or take them into account. In order to find that out, one question in the questionnaire asked the respondents whether their own organizations have any process relevant to OSRs or do not (see question 9 in Appendix A). The answers of the question were simply "no" or "if yes, please describe it". The person is given the chance to describe his/her own process in case he/she has a process.

As shown in Figure 14, about $73.6 \%$ of respondents said that they have NO requirements engineering process that takes into consideration OSRs. Whereas, $26.4 \%$ of the respondents claimed they have such a process and described it either in details or in short. Only 3 people skipped this question and did not answer it.

In total, 57 descriptions of different processes were received and analyzed. Not all of the descriptions were clear, or were actual representatives of processes. Therefore, after the analysis of the processes' descriptions, they were categorized according to similarity of the processes to each other. The following bullets summarize the processes provided by the respondents after the analysis.

- 19 respondents identify OSRs through requirements or specifications reviews in different ways such as peer reviews, reviews with stakeholders, or periodical reviews of requirements.

- 6 respondents reported to use tools support to find OSRs using requirements state or marking requirements as "obsolete", "deprecated". Furthermore, 2 respondents are using their own tools in which they defined their own processes in it.

- 6 people reported finding OSRs through requirements traceability. 
- Agile development was mentioned 4 times as the way to find OSRs through requirements priority and discussions with clients.

- 4 people assign a status or mark requirements as "obsolete" or "deprecated". They did not specify whether this approach is followed using a tool or a document.

- 4 people find OSRs through the verification and validation processes.

- 3 responses find OSRs during the requirements management process by identifying them.

- 3 people said that they move OSRs into a separated section in the SRS.

- 2 responses claimed that OSRs are part of their change management process.

- One response suggests that OSRs is part of the requirements analysis process.

- One person claimed having his/her own proprietary process.

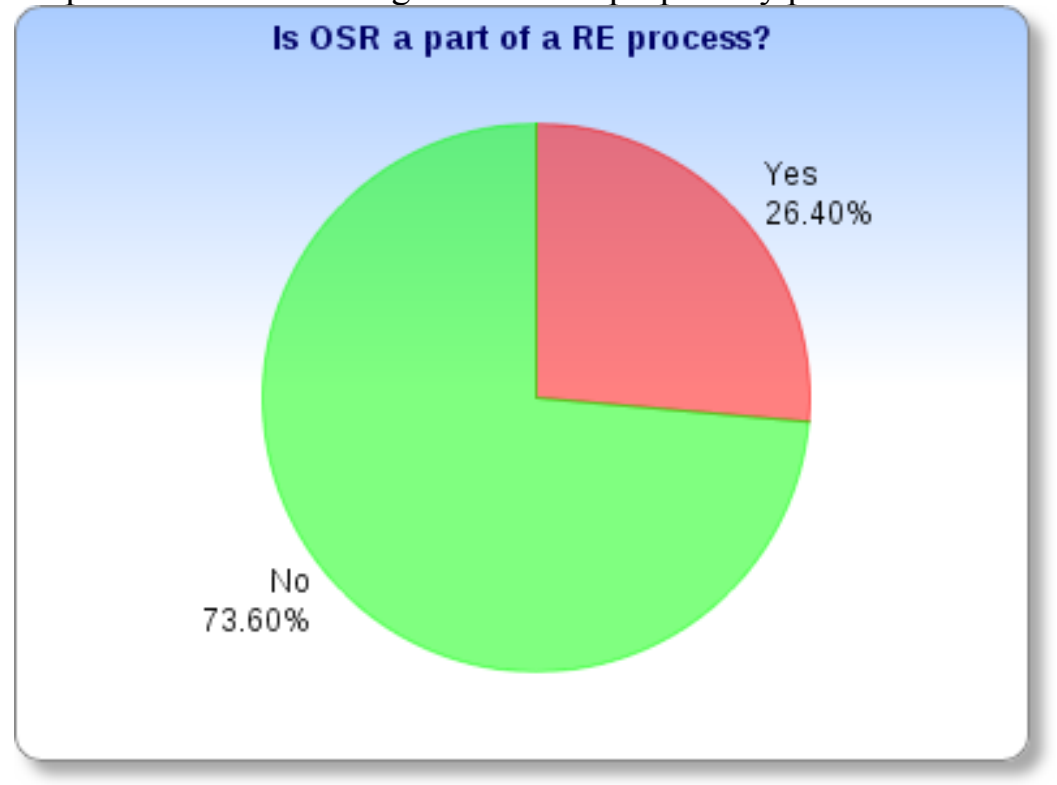

Figure 14: Is OSR a part of a process?

Other responses provided names or descriptions of methods used. They are summarized below.

- One person uses a method called "projective analysis".

- One person uses Hierarchical Requirements Table.

- One person uses the method of project governance.

- Inclusion of requirements tracking with risk management.

- "The requirements-based test plans are developed at the same time as, or ahead of, development", one respondent said.

- Commenting obsolete code and updating the requirements document accordingly.

- One person describes his/her process: "Use reqs validation techniques to identify reqs that are no longer needed. Use reqs traceability data (if you have it! almost no one does) to determine when a change (e.g., in a business rule) renders an existing req obsolete. Change the status of such reqs to "obsolete" and describe why. Retain the obsolete reqs, as they may come back into scope some day, but make sure there is no confusion among the developers about whether they are to be implemented or not." Note: reqs means requirements.

The reported experiences from the respondents show that reviews of requirements in different forms (19 responses), usage of tools (6 responses), that help retrieving and 
searching for OSRs, and requirements traceability (6 responses), are the most common used approaches/methods to find OSRs in the industry.

Moreover, further statistical analysis is conducted in order to find different relationships between the existence of requirements engineering processes that take OSRs into account (variable 1) and other factors such as respondents' roles, the size of organizations, and the development methodologies, (variable 2 each).

Section 2.2.3 reported that researchers and academician make $11.5 \%$ of the total percentage of respondents, and requirements specialists make a quarter of the respondents (see Figure 4 in section 2.2.3 for more information). There might exist a significant relationship between the respondents roles and the existence of RE process that takes OSRs into account (alternative hypothesis $\mathrm{H1}$ ). To find that out, chi-square test of significance was run on the data using SPSS.

The results of the chi-square test (Table 13) show that there is a significant relationship between the two variables $\left(\mathrm{X}^{2}=25.485, \mathrm{df}=8, \mathrm{p}=0.0012<0.05\right)$. Thus, the alternative hypothesis $H 1$ is not rejected. Moreover, there is a moderate association (Cramer's $\mathrm{V}=0.345$ ) between the respondents roles and the existence of $\mathrm{RE}$ processes that take OSRs into account.

Table 13: Chi-Square test for respondents roles and RE processes

\begin{tabular}{|l|l|l|l|}
\hline & Value & df & Asymp. Sig. (2-sided) \\
\hline Pearson Chi-Square & $25.485^{\mathrm{a}}$ & 8 & 0.00 \\
\hline Likelihood Ratio & 28.57 & 8 & 0 \\
\hline Linear-by-Linear Association & 0.35 & 1 & 0.56 \\
\hline N of Valid Cases & 214 & & \\
\hline
\end{tabular}

a. 4 cells $(22.2 \%)$ have expected count less than 5 . The minimum expected count is 3.08 .

It is worthwhile noting that chi-square test requires at least $20 \%$ of the cells to have expected count less than 5. However, in this case the roles 'system integrator' and 'software maintainer' received 3 and 2 responses, respectively. These two categories were merged with the 'others' category so as to reduce the number of cells that received expected count less than 5, and allow better and more realistic results. It is obvious from the note beneath Table 13 that $22 \%$ of the cells have expected count less that 5 . This does violate one of chi-square conditions, but the percentage is not high, and is tolerable. Moreover, the significance value is high $(p=0.0012)$.

In order to find the relationships between the respondents roles and the RE processes that take OSRs into considerations, a cross-tabulated result (also called contingency table) is presented in Table 14. The numbers in Table 14 without the parentheses are the count of the responses whereas the percentage of the responses from the overall percentage is presented in the parentheses of each cell.

The results in Table 14 show that requirements specialists have the highest percentage amongst others $(7.9 \%)$ who possess RE processes that take OSRs into account. Requirements specialists as their name suggests, work closely with requirements. Thus, their awareness of OSRs is higher than others. This is followed by software projects managers $(4.7 \%)$, and software team leader $(2.3 \%)$ (note here that 'Others' category comes in the second place, but this is not considered one role as it includes several roles, thus, it is not considered in the second place).

Surprisingly, academicians and researchers are amongst the lowest groups (i.e. developers, and testers) who own RE processes that take OSRs into account. Only one academician/researcher out of 25 (i.e. $0.5 \%$ ) reported that he/she is using a recent method called "projective analysis", and pointed out its reference (Cohen and Boxer, 'Why Critical Systems Need Help to Evolve', IEEE Computer, May 2010, pp56-63). 
Academicians and researchers mainly publish articles, write books about their topics more than practitioners do. So, it is obvious here that academicians and researchers are not aware of OSRs, and this justifies why there is a very few number of articles and publications mention OSRs (see section 3.3).

Table 14: cross-tabulation between respondents' roles and existence of RE process

\begin{tabular}{|l|lll|}
\hline Role & No & Yes & Total \\
\hline Software project manager & $12(5.6 \%)$ & $10(4.7 \%)$ & $22(10.3 \%)$ \\
\hline Software product manager & $10(4.7 \%)$ & $2(0.9 \%)$ & $12(5.60 \%)$ \\
\hline Software team leader & $11(5.1 \%)$ & $5(2.3 \%)$ & $16(7.5 \%)$ \\
\hline Software architect/designer & $19(8.9 \%)$ & $3(1.4 \%)$ & $22(10.3 \%)$ \\
\hline Requirements specialists & $37(17.3 \%)$ & $17(7.9 \%)$ & $54(25.2 \%)$ \\
\hline Developer/programmer/coder & $16(7.5 \%)$ & $1(0.5 \%)$ & $17(7.9 \%)$ \\
\hline Software tester & $11(5.1 \%)$ & $1(0.5 \%)$ & $12(5.6 \%)$ \\
\hline Researcher/academician & $24(11.2 \%)$ & $1(0.5 \%)$ & $25(11.7)$ \\
\hline Others & $19(8.9 \%)$ & $15(7 \%)$ & $3415.9 \%)$ \\
\hline & & & \\
\hline Total & $159(74.3 \%)$ & $55(25.7 \%)$ & $214(100 \%)$ \\
\hline
\end{tabular}

Furthermore, $53.7 \%$ of the questionnaire's respondents (the majority) work for large organizations (over 501 employees), and only $12.5 \%$ belong to small organizations (less than 10 employees), (see Figure 6 in section 2.2.3). The author hypothesizes that the size of the organization is related to the presence of RE processes that take OSRs into account (alternative hypothesis H1), whereas the null hypothesis (HO) suggests that there is no significant relationship between the organizations size and the presence of RE processes that take OSRs into account.

Table 15: Chi-Square test for size of organizations and RE processes

\begin{tabular}{|l|l|l|l|}
\hline & Value & df & Asymp. Sig. (2-sided) \\
\hline Pearson Chi-Square & $4.759^{\mathrm{a}}$ & 6 & 0.58 \\
\hline Likelihood Ratio & 5.22 & 6 & 0.52 \\
\hline Linear-by-Linear Association & 0.64 & 1 & 0.42 \\
\hline N of Valid Cases & 213 & & \\
\hline
\end{tabular}

a. 5 cells $(35.7 \%)$ have expected count less than 5 . The minimum expected count is 2.84 .

The results of chi-square test of significance is shown Table 15, it is clear that the null hypothesis $(\mathrm{HO})$ is not rejected $\left(\mathrm{X}^{2}=4.759, \mathrm{df}=6, \mathrm{p}=0.58>0.05\right)$ because the significance value $\mathrm{p}=0.58$ is greater than 0.05 . Additionally, $35.7 \%$ of the cells have expected count less than 5 which violates one of the chi-square test conditions. As a result, the alternative hypothesis $H 1$ is rejected. So, there is no significant relationship between the size of the organization and the presence of RE processes that consider OSRs in their phases. Even though many organizations with over 501 employees ought to have proper software development processes, and many of them would have CMMi certificates.

On the other hand, $23.6 \%$ of respondents adopt agile methods in their work while $17.1 \%$ of respondents use waterfall methods (see Figure 8 in section 2.2.3 for more results). In general, agile methods welcome changes of requirements at anytime during 
the course of the project [53] whereas waterfall methods mainly address requirements changes during the requirements phase in the SPLC. In addition to, other software development methodologies treat requirements changes in different aspects (the differences amongst development methodologies is beyond the scope of this master thesis). Therefore, the author hypothesizes that (alternative hypothesis H1) there is a significant relationship between the development methodology and the existence of requirements engineering processes that take OSRs into consideration. On the other hand, the null hypothesis (HO) suggests that there is no significant relationship between the development methodology and the existence of requirements engineering processes that take OSRs into consideration.

The result of the chi-square test of significance is $\left(\mathrm{X}^{2}=18.247, \mathrm{df}=10, \mathrm{p}=0.051\right.$ $>0.05)$. It is obvious that the significance value is larger than 0.05 , but it is only with 0.001 and is very closed to 0.05 . Thus, the null hypothesis (HO) is rejected and $H 1$ is accepted. As a result, there is a significant relationship between the development methodologies and the existence of RE process that take OSRs into account. Moreover, the development methodologies variable excluded the category "others" so as to reduce the number of cells that have expected value less than 5 . This is one of chi-square conditions that might invalidate its results (see section 2.3.2).

Looking at the column labeled "Yes" in the contingency table (Table 16), 23.3\% of the total respondents who answered "yes", they have processes that take OSRs into account are adopting agile methods as their software development methodology. On the other hand, $15.6 \%$ of the respondents that have processes that take OSRs into account use waterfall methods. Clearly, agile practitioners awareness of OSRs is more than any other practitioners of other software development methodologies. This awareness is ascribed to the nature of agile methods. Agile methods address requirements changes at anytime during the course of the project. Besides, in agile development, requirements creep on a monthly average of 10\% [5].

Table 16: contingency table between dev methodologies and RE processes.

\begin{tabular}{|l|l|l|}
\hline Development methodology & No & Yes \\
\hline Agile & $24.90 \%$ & $23.30 \%$ \\
\hline Incremental/evolutionary & $20.30 \%$ & $16.70 \%$ \\
\hline Waterfall & $18.50 \%$ & $15.80 \%$ \\
\hline Prototyping & $7.80 \%$ & $12.50 \%$ \\
\hline Rational Unified model & $7.50 \%$ & $8.30 \%$ \\
\hline Components-based software engineering & $5.00 \%$ & $8.30 \%$ \\
\hline Ad hoc (no method) & $6.80 \%$ & $1.70 \%$ \\
\hline Rapid application development. & $3.90 \%$ & $5.00 \%$ \\
\hline Spiral development. & $2.50 \%$ & $5.80 \%$ \\
\hline Formal methods & $2.80 \%$ & $2.50 \%$ \\
\hline Total & $100.00 \%$ & $100.00 \%$ \\
\hline
\end{tabular}

At the same time, agile practitioners who have "NO" RE process that take OSRs into account have topped the list with $24.9 \%$ of the total percentage, as opposed to $18.5 \%$ of waterfall methodology practitioners. The reader can see all the percentages in Table 16. 


\subsection{Suggested processes for obsolete software requirements}

There is no one standard or unique requirements engineering process neither in the industry nor in the literature. Usually, the organizations either develop their own requirements engineering process or follow a certain process that is common in the industry or literature. Besides, some organizations follow an ad hoc (no method) methodology for their requirements engineering stage and/or for the whole project lifecycle. Therefore, this section suggests ways or processes that could be used by organizations to find/detect OSRs regardless of the followed processes in the organizations.

There are two ways to manage requirements. The first one is usually done using word documents or excel sheets (this is usually practiced by small and medium organizations). The second way is using a requirements management tool which is usually database driven (large and very-large organizations most likely use such tools) [1], [2]. The suggested processes -by the author- will cover both ways and they will be called in this thesis "document-based requirement" (section 5.2.2) and "database-based requirements" (section 5.2.3), respectively. But before describing the suggested processes, there are some assumptions that organizations should have (section 5.2.1).

In general, the generic form of the proposed processes will be divided into two parts, the first part is how to find OSRs, and the second one is what to do about them after they are discovered. The questionnaire contains two separated questions for the two parts, respectively.

Question 4 in 'Appendix A' suggests three ways of discovering OSRs i.e. reading all requirements manually, using built-in search functionalities that look for certain attributes, or having a predefined status called obsolete. Also, the respondents could write down their own suggestions or processes. In addition to, a choice was given which states that the person never thought of OSRs or discovered them before.

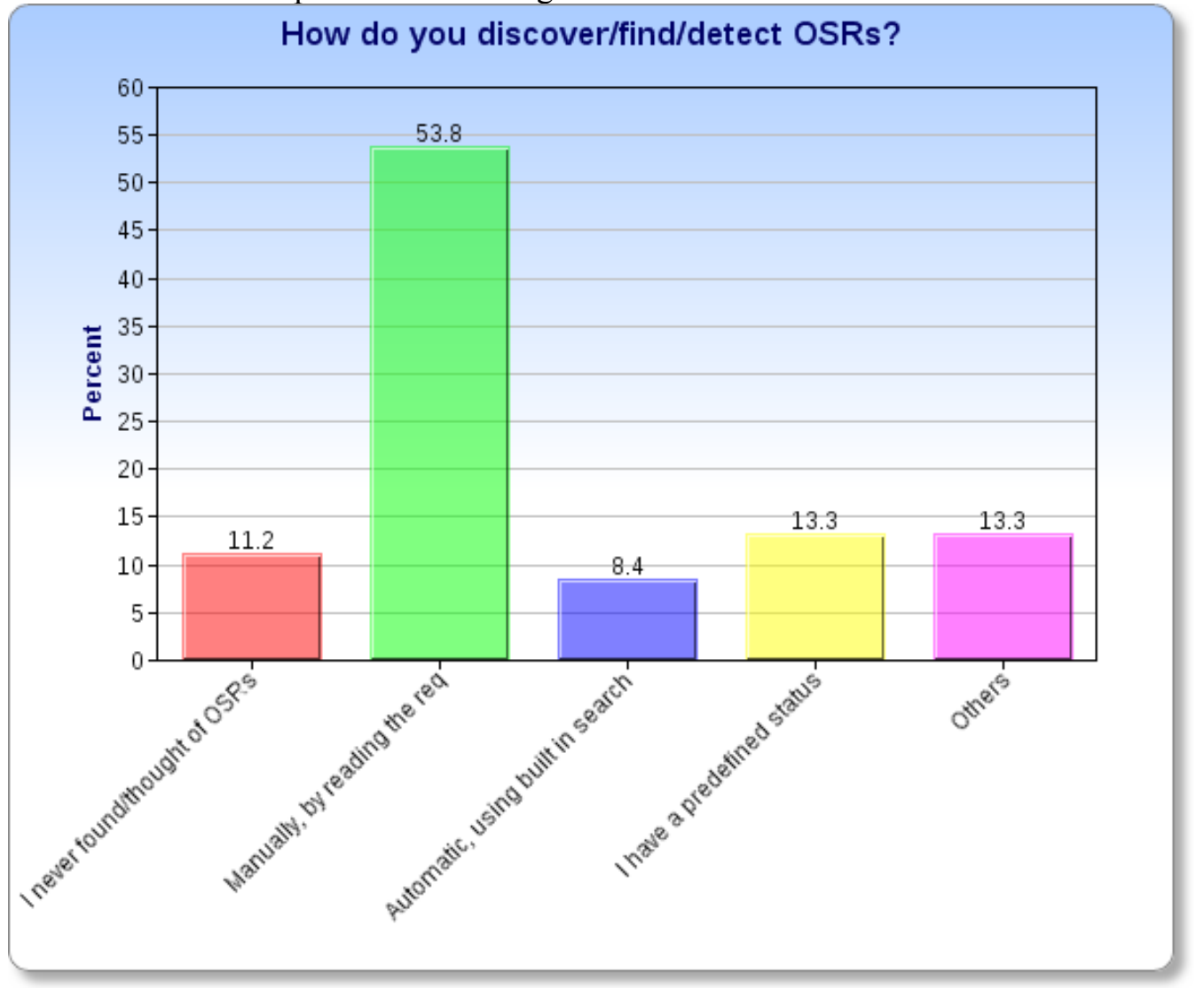

Figure 15: Ways to find/detect OSRs 
Figure 15 shows that the majority of participants (53.8\%) agrees on reading the requirements manually, and after identifying the OSRs mark them as obsolete. This is followed by having a predefined status called obsolete, however, only $13.3 \%$ voted for this choice. Then the use of built-in search functionalities comes in the third place recording only $8.4 \%$. It is not surprising that about $11.2 \%$ of respondents never found or thought of OSRs (see section 5.1). Even though this is a small percentage, this is an indication that OSRs are still absent in practice for a number of respondents.

Moreover, around $17.4 \%$ of responses were suggesting different ways of the defined ones i.e. "others" category. Nevertheless, the answers were not new and many of them were similar to the points mentioned in section 5.1. Note that only 1 person skipped this question.

In order to find statistical relationships between the ways to discover OSRs and the respondents roles, the size of organizations, and the development methodologies, respectively, further statistical analysis using chi-square test of significance (see section 2.3.2) have been conducted as shown below.

Chi-square test of significance was run on the data of "ways to discover OSRs" and the respondents roles. The results of chi-square test in this case are considered invalid due to the violations of chi-square conditions namely: there are more than $20 \%$ of cells that have values less than 5, and more importantly, many cells have values less than one. Therefore, there is no significant relationship between the ways to discover OSRs and the respondents roles.

Similarly, there is no significant relationship between the "ways to discover OSRs" and the size of the organizations $\left(\mathrm{X}^{2}=29.443\right.$, $\left.\mathrm{df}=30, \mathrm{p}=0.494>0.05\right)$. Besides, more than $20 \%$ of cells have expected cell counts less than 5 . Thus, chisquare results may be invalid.

Finally, there is no significant relationship between the "ways to discover OSRs" and the development methodologies because the available data does not meet two conditions of chi-square test i.e. the values in the cell should not be less than one, and there should be less than $20 \%$ of the cells with values less than 5 .

Question 5 in 'Appendix $\mathrm{A}^{\prime}$ is about what to do with OSRs after they are discovered. There are four actions suggested by the author, and the respondents could add their own actions. The actions are:

1) Deleting the requirement.

2) Keeping it and assign it a status called obsolete.

3) Keeping it and assign it a status called obsolete and provide the rationale behind making a requirement obsolete.

4) Moving all OSRs into a different section in the document.

Figure 16 shows that choice number 3 "keep the requirements and assign a status called obsolete and provide the rationale why a requirement became obsolete" has topped the actions with $54 \%$ of votes. It was followed by "moving OSRs into a different section in the document" with $21.9 \%$ of responses. Only $8.9 \%$ favored choice number 2 i.e. keep the requirements and assign a status called obsolete. Unsurprisingly, some people want to delete the requirements as they consider them valueless and not supposed to remain in the documentation. However, these are the smallest group and they only make $8.6 \%$ of the responses.

Moreover, 20 responses $(6.6 \%)$ were "others" category and the respondents have specified their own action. The following bullets summarize the analysis of the answers:

- Three people suggested using advanced features of tools. Defining a status like "obsolete" to the requirements and then hide/show them.

- Keeping requirements and not to delete them at all because they might be implemented in previous releases, three respondents said.

- One person proposed moving all OSRs into a separated document. 
- One person uses version control of requirements, in this way, he/she retains all previous versions of any requirement, and does not need to have a status.

- Removing OSRs during a special session conducted for that purpose, one person describes his/her own way of handling OSRs.

- A couple of respondents deal with OSRs throughout the code and test cases. Indeed, their processes sounds similar to some extent to the process in [26].

- Seven responses were not clear and did not represent any action to be taken against OSRs such as this "We use Rejected and Deleted. Deleted is in case a requirements was accidentally added."

It is worthwhile noting here that 218 people replied to this question and only one respondent skipped it.

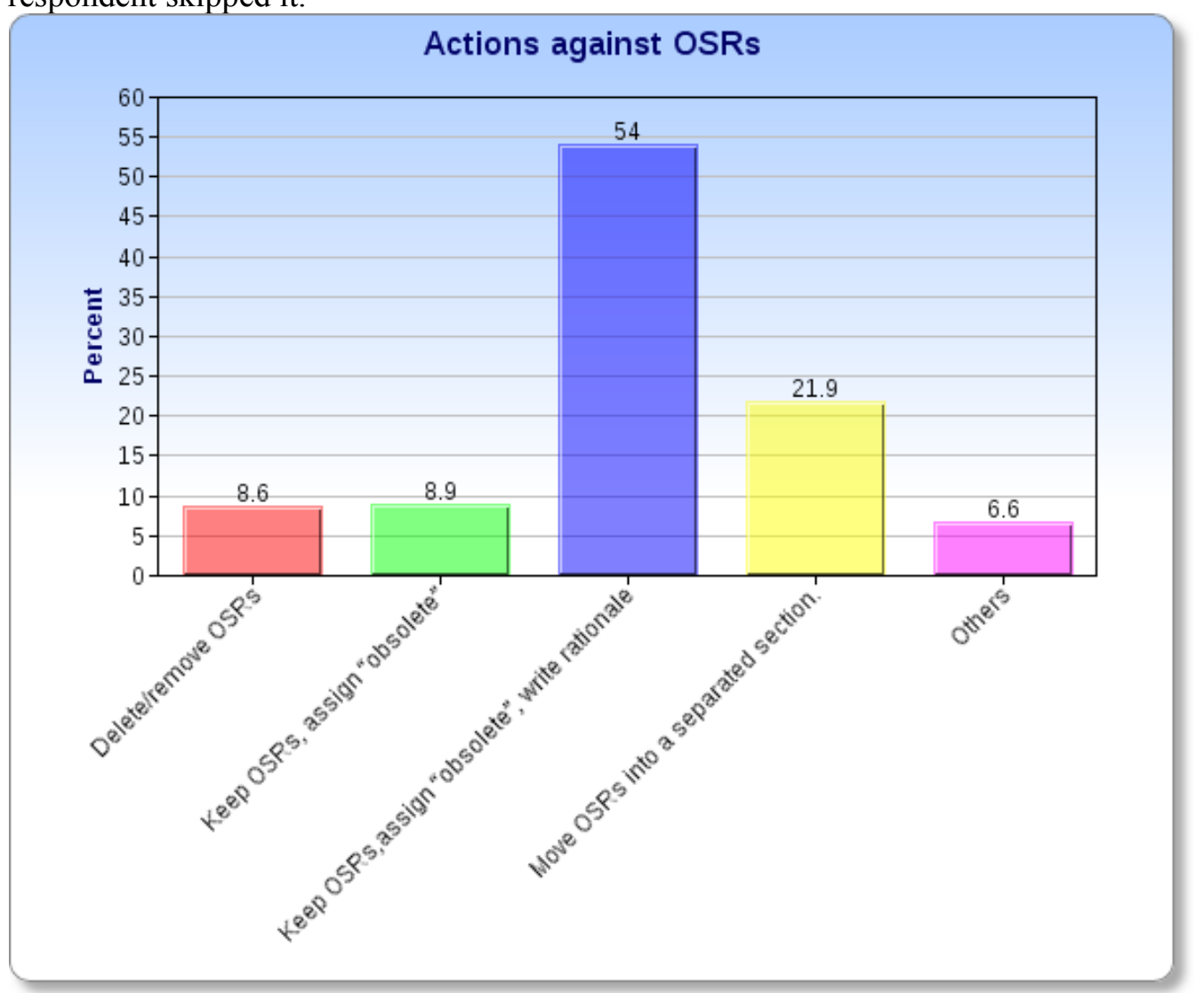

Figure 16: Possible actions against OSRs

In the same manner, as done earlier, the author attempts to find statistical relationships between the possible actions to be taken against OSRs after they are discovered on one side, and the respondents roles, the size of organizations, and the development methodologies, respectively, on the other side. To do that, chi-square significance test have been conducted. The results (shown below) revealed that there is no significant relationships between "actions against OSRs" and the respondents roles, the size of organizations, and the development methodologies, respectively.

The chi-square test result of the respondents roles and "actions against OSRs" is $\left(\mathrm{X}^{2}=33.163, \mathrm{df}=40, \mathrm{p}=0.769>0.05\right)$. As well, more than $20 \%$ of cells have expected cell counts less than 5 . Thus, chi-square results may be invalid.

Likewise, the chi-square test result of the size of the organization and "actions against OSRs" is $\left(\mathrm{X}^{2}=37.928, \mathrm{df}=50, \mathrm{p}=0.895>0.05\right)$. In addition, more than $20 \%$ of cells have expected cell counts less than 5 , and the minimum expected count of some cells is less than one. Therefore, chi-square results may be invalid. 
Finally, the results of chi-square test between the "actions against OSRs" and the development methodologies is invalid due to the violations of chi-square test conditions. The violations are: more than $20 \%$ of cells have expected cell counts less than 5, and many cells have minimum expected cell count of less than one. However, the reported results of SPSS are $\left(X^{2}=71.015, \mathrm{df}=50, \mathrm{p}=0.027<0.05\right)$, this results is significant at the 0.05 level. But, the result is rejected due to the violations of chisquare conditions as mentioned earlier.

\subsubsection{Assumptions}

The author assumes that software organizations have certain basic elements of requirements management (described below). Usually, each organization has its own requirements management policies, or follows certain standards. However, the following points present the least that -the author assumes- should be done by any organization to manage their requirements.

- Since requirements creep monthly [5], there should be a way to manage what to include or exclude in the product. This is usually done through dividing the product into a number of releases that will be produced in the future [54]. Thus, the requirements that are considered for development belong to one release only in a product of several releases.

- There is a requirements change process/policy in the organization.

- The requirements engineer, or the Change Control Board (CCB) take the decision of including or excluding requirements in the release. It could also be that this decision is taken as an agreement between the customer and the developer. The abbreviation CCB will be used to describe that role in the rest of this section.

- Every requirement is uniquely identified.

- Every requirement has a requirement status.

- The requirements that are used for one release are put into a document/file and it is called the requirements baseline. It is "the set of functional and nonfunctional requirements that the development team has committed to implement in a specific release" [55].

- Most of the requirements are written in a natural language, for example, English.

\subsubsection{Document-based requirements.}

Changes in requirements are common and changes lead to volatility. In its turn, volatility leads to obsolescence [14]. Thus, it is advisable to maintain a list of volatile requirements in order to keep track of them [56]. This list could be made in several forms based on the organization's policies and standards such as a table containing the requirements IDs of the volatile requirements, or creating a requirement status called "volatile", or assigning a value to each requirement to indicate its volatility, or anything else that indicates that the requirement is very changeable.

Indeed, maintaining such a list makes it easy to the CCB to identify potential requirements that might become obsolete by the next release. The CCB should first start by reading the list of volatile requirements if they exist, and then read the rest of the requirements. Otherwise, they have to read the whole document without paying attention to the types of requirements, and to specify the volatile requirements and/or obsolete requirements.

The questionnaire conducted along the master thesis asked the people how would they discover/find/detect OSRs in a software requirements document or database. As shown in Figure 15, 70.6\% of the responses are with the choice "Manually, by reading the requirements specification and marking requirements as obsolete".

If the organization maintains volatile requirement statuses, it is advisable to start reading those requirements and decide which one has become obsolete or has not. Additionally, if the organization does not have a dedicated status to denote "obsolete" 
or "deprecated" requirements, it is advisable too to start having such a status. The latter status helps in retrieving OSRs faster and easier. Figure 15 shows that only $17 \%$ of respondents have a predefined status called "obsolete". One concludes that OSRs are not widely recognized by many organizations.

After determining the OSRs, many would think that those requirements are supposed to be taken out or deleted from the requirements document $(11.9 \%$ only, see Figure 16). On the contrary, researchers agree on keeping them with the rest of requirements and writing the rationale behind making the requirements obsolete [13,34,39-41]. This opinion has been confirmed by the results of the questionnaire, $74.9 \%$ of responses agreed on keeping the requirements, on assigning them a status called "obsolete", and on writing the reason why they become obsolete for future reference (database/document). The motivation behind keeping OSRs in the documentation is for future reference i.e. CCB or any stakeholder can refer to them back in the future and see why such requirements became obsolete so as to avoid implementing them again.

Moreover, in the case of document-based requirements, there are different actions that could be taken against OSRs:

1. Keeping the requirements in their place adding extra status called "obsolete" (12.3\% of responses, Figure 16$)$.

2. Keeping the requirements in their place striking them out tike this. Or;

3. Moving all OSRs into a separated section calling it "obsolete requirements", or whatever name one finds meaningful (30.1\% of responses, Figure 16$)$.

To summarize, the majority of responses agrees on reading the requirements manually, and on deciding which requirements have become obsolete or have not, and on assigning a status to the requirements providing the reason for making them obsolete.

\subsubsection{Database-based requirements}

It is important to manually read all the requirements before deciding on what requirements to include/exclude for the next software release (baseline). However, the use of requirements management tools allows for more functionality to search for requirements, categorizes them, identifies them, etc. There is a plethora of requirements management tools in the market (the difference among them and their names are beyond the scope of the thesis. Look at [34] for further information).

This section provides several possible ways of finding/discovering OSRs and treating them after being discovered. The reader can decide which way suits his/her environment, or he/she can use more than one way. It is not possible to propose one process for all organizations and requirements management tools because there are plenty of them. As well, software organizations have different ways of handling requirements such as having requirements status, prioritizing requirements, requirements traceability, version control of requirements changes, to name a few.

\section{Possible ways of discovering OSRs.}

Tools with version control support:

Many requirements management tools provide version control support. Every time someone makes a change on a requirement, its old version will be stored and the latest version (after the changes) will be displayed to the person. In addition to, one can view all the previous versions of a requirement and can rollback to any version. Utilizing version control to find/discover OSRs in this manner is a viable method. The number of versions of requirements indicates how volatile a requirement is. So, by ordering requirements (ascending/descending) based on the number of versions of each one, it is possible for the CCB to find/detect OSRs first. 
This solution might be feasible for many software organizations because many of the requirements management tools support version control of requirements. Moreover, software organizations which use commercial requirements management tools have no control over changing the functionalities of the tools and their internal source code. So, it might not be possible for many organization to view requirements based on the number of versions of each requirement. On the other hand, organizations which develop and use their own requirements management tools are able to add the functionality of viewing requirements based on the number of versions of each requirement.

Requirement Status/State:

It is a core feature of any requirements management tool to have requirement status. It is advised by the researchers, as well [2], [55], [56]. Usually, the requirement status is predefined by organizations according to their process. Nevertheless, there are common statuses as rejected, deleted, approved, etc. It is advised to start having a status called "obsolete" to indicate that the requirement is outdated. In this way, one can always view/read requirements based on their status. So, in order to find OSRs, one might want first to read requirements which have statuses that indicate they are volatile or changeable.

Creating a new status/state is usually allowed by commercial requirements management tools. Thus, this way of tagging requirements is feasible by software organizations, and does not require a lot of efforts to implement.

Not implemented requirements:

Many of software requirements are not implemented for various reasons. They only live in papers or in the specifications document. If there is a status/state which says that a requirement has been implemented or has not, one can view all requirements which have not been implemented yet, or have been delayed for various reasons. Some of these requirements might have already become obsolete and should be treated like so. In case there is no status to determine if a requirement is implemented or is not. It is a good idea to consider having it so in order to ease the requirements management process.

Viewing implemented requirements and not implemented requirements is viable by requirements management tools. What one needs here is to create a new status/state for that purpose. So, software organizations that use commercial requirements management tools or in-house developed requirements management tools can simply add this status/state.

Requirements priority / Mandatory and optional requirements:

It is a good practice to assign priority for each requirement [56]. The priority value indicates to the software managers or developers how important a requirement is to the customer or to the success of the project. One can assign different scales of priority, or one can use a mandatory/optional scale to tell that a requirement is a 'must have' or it is a 'nice to have' respectively. This depends on the available resources and decided by CCB. Regardless of the used scale, the low priority requirements, or the optional requirements are most likely to become obsolete over the course of time. In order to find potential OSRs, it is advised to start reading the low priority or optional requirements first, then to read the higher level in the priority scale, and so on.

The requirements priority notion is very common in agile development. As well, most of the modern requirements management tools support it [2], [56]. So, it is feasible by software organizations to implement this strategy to find OSRs. In case the software organization is using home-made requirements management tool, it is not difficult to add this feature to the tool.

Volatility value:

The volatility of requirements differs. As well, the volatility degree or value is not the same for all requirements. For example, some requirements might only change 
once while others might change five times. Therefore, it is a good idea to assign a volatility value which describes how much volatile a requirement is. Sommerville suggests using a scale from 1 to 3 where "1 means fairly stable, 2 means subject to medium term change, and 3 means that the requirement is likely to change in the shortterm" [56]. Indeed, one can have his/her own scale and assign different meanings to different values. What is essential here is the fact that the volatile requirements should be checked first because they are most likely to become obsolete first [14].

This solution might be infeasible by many software organizations because the concept of requirements volatility is relatively new. Moreover, few researches have been conducted on it. In case software organizations have commercial requirements management tools that do not support requirements volatility degree, it is not possible to use this strategy to discover OSRs. While in the case that software organizations have their own requirements management tools, it is not difficult to implement the volatility value functionality in the tool.

Requirements types:

There are several classifications of requirements as shown in section 4. As mentioned earlier, volatile requirements have several degrees of volatility, for that reason, it is a part of this master thesis to find the relationship between the requirements types and volatility (section 4.1). In case you do not assign to the requirement its type (e.g. hardware requirement, standard requirement), it would be a good idea to do that, because, first, you can know the nature of the requirement without having to read it, and, second, you could know if the requirement is volatile and/or becoming obsolete or is not. See section 4.1, for more information about requirements types and the relationships to obsolescence.

Utilizing the concept of requirements type to discover OSRs might not be feasible and face several challenges. In practice, there is no one agreed-on classification of requirements. Moreover, software organizations adopt different requirements classifications, or they develop their own classification. Nevertheless, if the requirements management tool used by software organizations support assigning attributes for requirements type, this method could be possible to discover OSRs. But, software organizations have to agree internally (at least) about a classification of requirements, and they have to standardize it. Otherwise, this method could create more confusion for requirements specialists.

Hybrid ways:

It is possible to make hybrid ways of the above mentioned methods. For instance, one can use the requirements status along with the requirement volatility degree to search for OSRs. Another possible hybrid way could be by looking at low priority, not implemented requirements, and the number of versions, in order to see if these requirements keep changing and have yet never been realized. However, other more ways are possible. They are up to the CCB and the organization's policies.

Moreover, the usage of hybrid ways of discovering OSRs depends heavily on the support of the requirements management tools. In case of commercial tools, it is not always possible to tune the tool according to your needs. But, in case of home-made tools or open source tools, it is possible for software organizations to tweak the tools and implement different ways of finding OSRs and treating them.

\section{Possible actions against OSRs}

Once OSRs are found, one has to take action against them. In database-based requirements, it is possible to keep the requirements in the database and then assign them a status called "obsolete" or something similar, to indicate that this requirement is obsolete. In addition to, it is strongly recommended to provide the reason why the requirement did become obsolete (see Figure 16 and see section 5.2). 
Other possible actions depend on the used requirement management tool. In case the tool allows different requirements views, one could simply hide OSRs from the view. Hiding OSRs means they are still stored in the memory but not shown to the reader in order to ease the reading of other requirements.

Even though deleting/removing any requirements is not advised as mentioned in section 5.2, it is still the option that around $11.9 \%$ of the respondents have chosen (see Figure 16).

In conclusion, the majority of respondents claim to have NO requirements engineering process that takes OSRs into account ( $73 \%)$. Interestingly, academician and researchers are reported to be the least who have requirements engineering process that takes OSRs into consideration. This, however, justifies the absence of literature from information about OSRs. Moreover, reading the requirements manually and trying to discover OSRs, as well as, has topped the ways of finding them. As well, retaining OSRs in the documentation, and assigning them a status such as obsolete, and writing the rationale behind why they became obsolete has been selected by the majority of the questionnaire's respondents as the way to treat OSRs after being found. In addition, this section suggests ways to find OSRs in the documentation (document and database based documentation) and ways to deal with them after they are discovered. 


\section{Obsolete Software Requirements Effects}

This section studies the effects of OSRs on software projects depending on the project size and the project type. This section answers research question number 5 in Table 1.

Theoretically, requirements volatility leads to requirements obsolescence [14]. Researches have shown negative effects of requirements volatility such as increasing the defects density [10]. However, the effects vary from project to project. For example, the size of the project (in terms of number of requirements) plays a role in increasing or decreasing the negative effects. The larger the project, the harder its management is. Moreover, the development methodology plays another role. To illustrate, the waterfall method is a rigid method and does not properly address requirements changes as opposed to agile methods that welcome requirements changes at anytime.

In this section, the influence of OSRs is studied from two directions. The first direction (section 6.1) is on the size of the project (e.g. large scale projects with thousands of requirements), and the other direction (section 6.2) is on the type of the software project (e.g. market-driven projects).

\subsection{Obsolete software requirements effects on the project size.}

In the web questionnaire, a question about the effects of OSR on the size of the project in terms of number of requirements was asked. The respondents were asked to rate how likely OSRs affect or impact the project based on its size i.e. small scale project (tens of requirements), medium scale project (hundreds of requirements), largescale project (thousands of requirements), and very-large scale project (over ten thousand requirements) [4]. The rating is on a scale from 1 to 5 where 1 is 'not likely' and 5 is 'very likely'.

The results could be viewed in two different ways. The first way is to study the average rate of responses (mean) for each project size category (Figure 17). However, the second format is to present all the responses for each category as is (Figure 18).

The results shown in Figure 17 represent the mean (average rate) for each project size. It is obvious that the results in Figure 17 conform with the common sense that says the larger the project the more difficult it is to manage. So, it is more likely that OSRs will affect the projects with larger number of requirements. In other words, the effects of OSRs (negative) on small scale projects (tens of requirements) are less than the ones on medium, on large, and on very-large scale projects. The average rate of responses (mean) is as follows: small scale projects 2.17 , medium scale projects 2.7 , large-scale projects 3.38 , and very-large scale projects 3.73 . Note here that 4 respondents out of 219 skipped this question.

On the other hand, Figure 18 shows all the responses for all categories and scales (1 to 5). The majority of respondents considers OSRs to have slight negative effects on small scale projects (ten of requirements). But, 'not likely' and 'somehow likely' categories received almost the same percentage of votes $35.3 \%$ and $35.8 \%$, respectively. In essence, the majority agrees that the effects of OSRs on small size projects are not considerable.

On the other hand, Figure 18 shows that, $41.5 \%$ of all respondents believe that OSRs are likely to affect medium scale projects (hundreds of requirements), while $31.6 \%$ believe that OSRs are somehow likely to affect medium scale projects. Likewise, $32.7 \%$ of respondents consider OSRs are 'more likely' to affect large-scale projects (thousands of requirements), while $31.3 \%$ think they are likely to affect largescale projects. Finally, about $39 \%$ deal with OSRs as a serious matter concerning verylarge scale projects (over ten thousand requirements). It is followed by $23.7 \%$ of 
respondents who think it is more likely that OSRs will severely affect very-large scale projects.

On looking at Figure 18, it turns out clear that the respondents consider very-large scale projects the most affected category by OSRs. Furthermore, there is about $16 \%$ gap between 'more likely' category and 'very likely' category. This gap shows that more respondents deem it as a serious matter regarding very-large scale projects.

In aggregate, OSRs are believed to have negative effects on large and very-large scale projects (i.e. over thousands of requirements) and practitioners and researchers should pay closer attention to OSRs phenomenon.

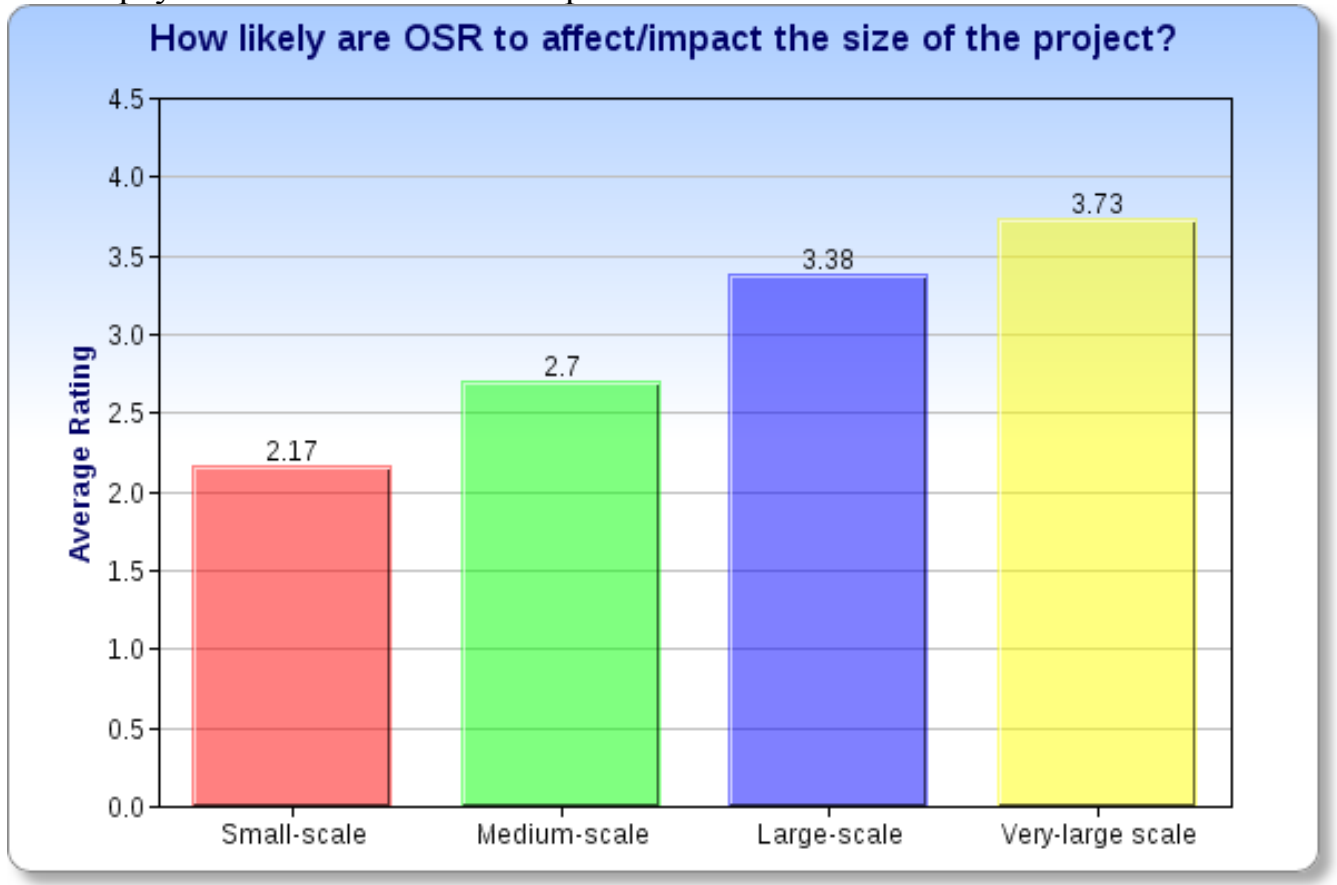

Figure 17: Effects of obsolete requirements on the project size

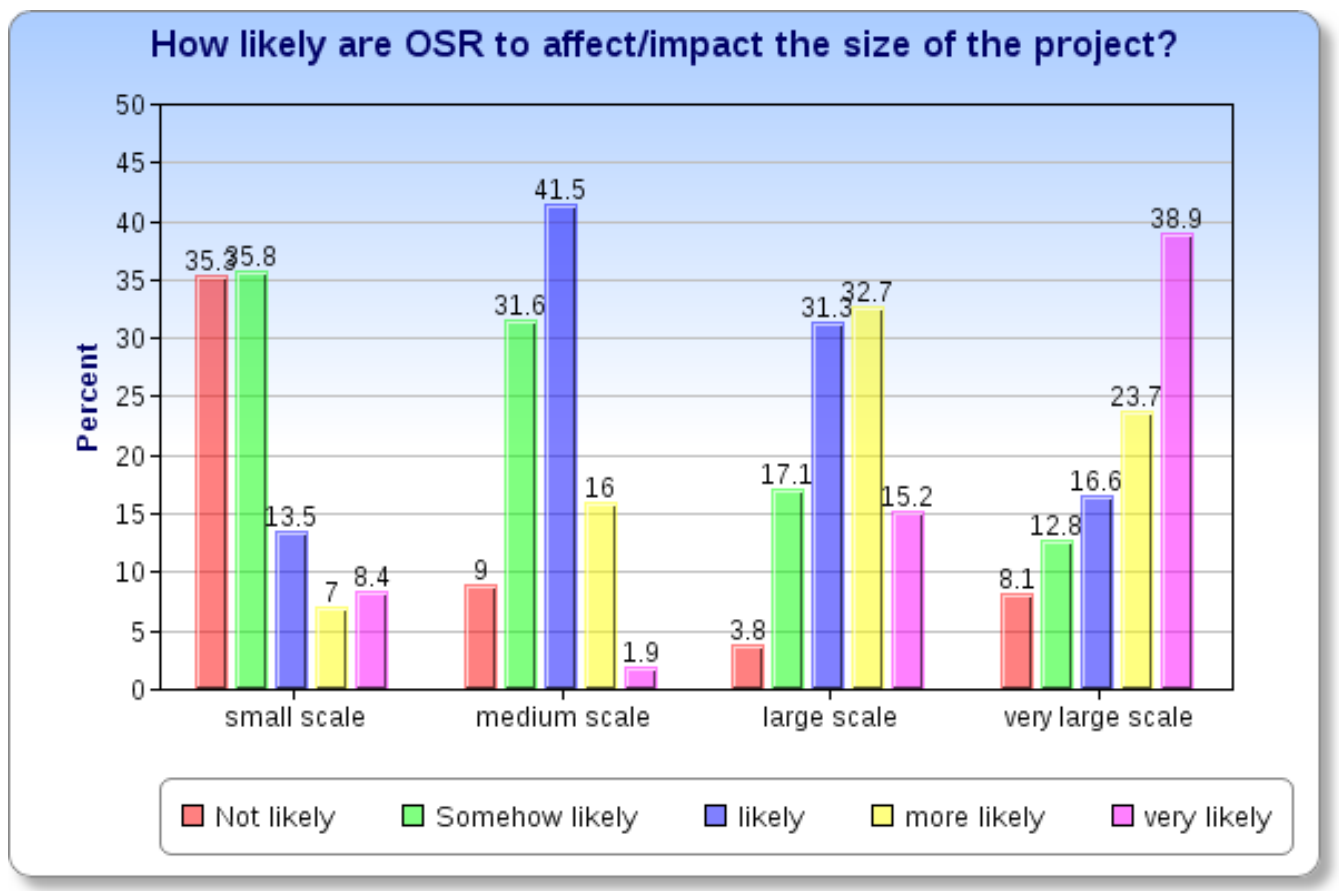

Figure 18: effects of obsolete requirements on the project size (all responses) 


\subsection{Obsolete software requirements effects depending on the type of the project.}

The questionnaire has a question about the effects of OSRs on the project type i.e. bespoke projects (software that is specially developed for some specific organization e.g. students portal for a specific university), market-driven projects (software that is made for the open market place e.g. office applications), and outsourced projects (software that is given to a third party to produce it). The respondents are asked to rate how likely the project type will be affected by OSRs on a scale from 1 to 5 where 1 is not likely and 5 is very likely.

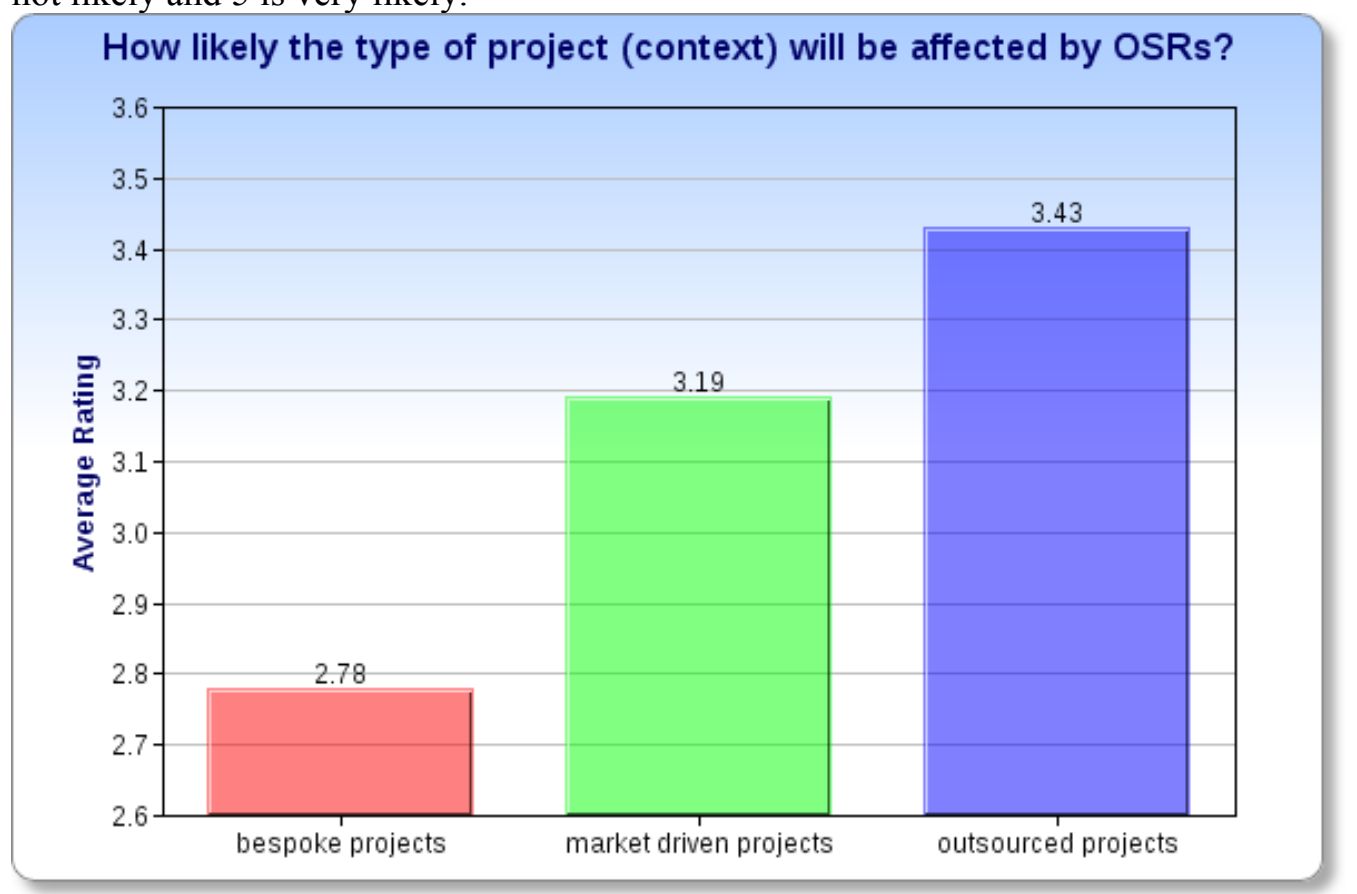

Figure 19: effects of OSR on the project type (average rate)

Like the previous section (6.1), the results can be viewed in two different formats. The first one is to study the average rate of responses (mean) for each project size category (Figure 19), while the other one is to present all the responses for each category as they are (Figure 20).

The average rate of responses (mean) (Figure 19) shows that outsourced projects are the most affected type of projects (3.43). Indeed, outsourced projects face more challenges and obstacles than other types of projects do. They are usually contracted to a third party organization to develop them. In many cases, the third party resides in a different location from that of the customer. As well, the third party might be spanned over several locations. Moreover, in their article Jyoti et al. show from their experience several cases of challenges in the context of outsourced and offshore projects. Conflicting goals of stakeholders, low client involvement, and conflicting RE approaches, are few examples of such challenges [58]. According to the results of the questionnaire (Figure 19 and Figure 20) OSRs would exacerbate the problems in outsourced projects, and would be considered another challenge added to the list.

Market-driven projects and bespoke projects have come in the second and third places with scores of 3.19 and 2.78 , respectively. It is obvious that the gap between market-driven driven projects and bespoke projects is wider than it is between outsourced projects and market-driven projects.

Nevertheless, more attention should be paid to market-driven projects than to bespoke projects due to the differences in the RE process between them. In his thesis, 
Awan [59] compared both types and found that market-driven projects are different because they need to be delivered to the mass market in short time and incrementally. Furthermore, the users expect such software to be almost error-free. Other factors influencing market-driven projects are: there is no customer involvement in the RE process, and requirements are usually invented by marketing departments and developers. This means that the requirements flow is larger than bespoke projects and takes more resources to manage. In addition to, requirements are more volatile than bespoke projects requirements and thus are more prone to become obsolete. Another difference that exacerbates the problem is that there is no contract written in marketdriven projects, and thus, many requirements are not documented.

In aggregate, OSRs should not be neglected in any project's type. However, more attention should be paid to outsourced projects and market-driven projects than to bespoke projects as they are deemed by the respondents to be more affected by OSRs.

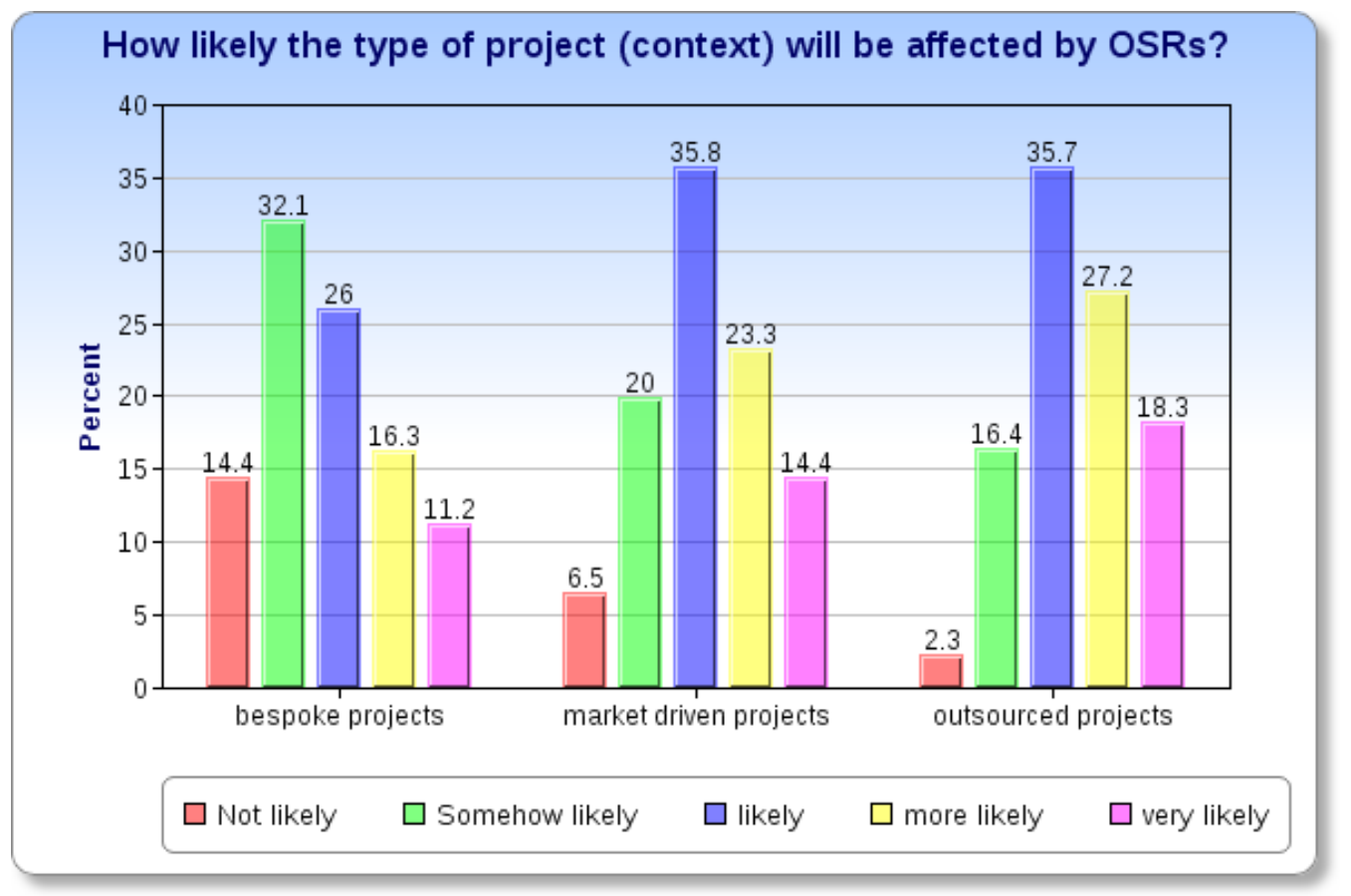

Figure 20: effects of OSR on the project type (all responses)

In conclusion, the results of the questionnaire revealed that OSRs can affect software projects. These effects vary based on the characteristics of the project. To illustrate, OSRs affect large and very-large scale software projects more than small and medium small projects. So, OSRs affect software projects based on their size (in terms of number of requirements). On the other hand, OSRs affect the type of the project (from the business perspective). The results show that outsourced software projects are the most affected type, followed by market-driven projects and finally bespoke software projects. 


\section{The Requirements Engineering Process and Obsolete Software Requirements}

This section proposes to insert/inject OSRs into the requirements engineering lifecycle, and into the requirements management process. This section answers research question number 6 in Table 1.

The requirements engineering life-cycle or process goes through 5 major phases: requirements elicitation, analysis, specification, validation and requirements management. All these processes are interleaved and not separated, and one might not have to go through them in order, or they might be done concurrently [1]. Moreover, requirements management comprises of requirements change, requirements status, requirements traceability, and requirements tracking [2]. Requirements prioritization and requirements volatility are considered sub-processes of other phases or processes in the requirements engineering life-cycle.

It is important to know where to inject/insert OSRs in the RE life-cycle so as to allow the requirements experts/specialists to focus on it on certain phases, though OSRs might be applicable in every phase.

The questionnaire contains a question about where to put OSRs in the RE lifecycle and/or in the software requirements management (see question 8 in Appendix A). A predefined list of the phases is provided and the respondents could choose more than one phase (multiple answers) and are allowed to write their own answers.

The respondents have chosen that requirements changes, requirements validation, and requirements analysis phases are the most important phases that one should pay attention to OSRs in. They received $14.8 \%, 14.6 \%$, and $14.5 \%$, respectively, as shown in Figure 21. It is also clear from Figure 21 that the gap between the top 3 phases and the rest ones is considerable. It is around 5\%. This does not mean that the other phases which received lesser votes are to be neglected. As mentioned earlier, RE phases are interconnected. So, one should pay more attention to OSRs during requirements changes, validation, and analysis phases.

Indeed, requirements change is connected with OSRs as change leads to volatility, and volatility, in its turn, leads to obsolescence [14]. Even though OSRs are a result of requirements volatility, requirements volatility received the lowest responses with $4.7 \%$ only. This would mean that both OSRs and requirements volatility are part of requirements changes. As a result, the requirements engineer has to look for OSRs mainly during the requirements changes process.

Furthermore, requirements analysis in which requirements engineers analyze the feasibility of implementing requirements, and requirements validation where requirements are checked for their sanity and correctness, are also strongly connected with OSRs.

Nevertheless, 27 responses (3.6\%) were given to the "others" category in which the respondents wrote other processes. After analyzing the responses, nine responses suggest that OSRs are discovered at anytime during the life-cycle of the project. Two respondents suggest that OSRs are discovered during testing and quality assurance time whereas one person specifies it during "implementation". Moreover, three answers were not clear.

What important here is that 11 respondents did not understand the question, and many of them mentioned the word "put" as unclear. It seems that the word "put" was vague to many readers. One respondent suggested using the word inject/insert instead. So, it is important to take the results of this question with extra care since 11 people out of 216 did not understand it, and only 3 people did not answer it. 


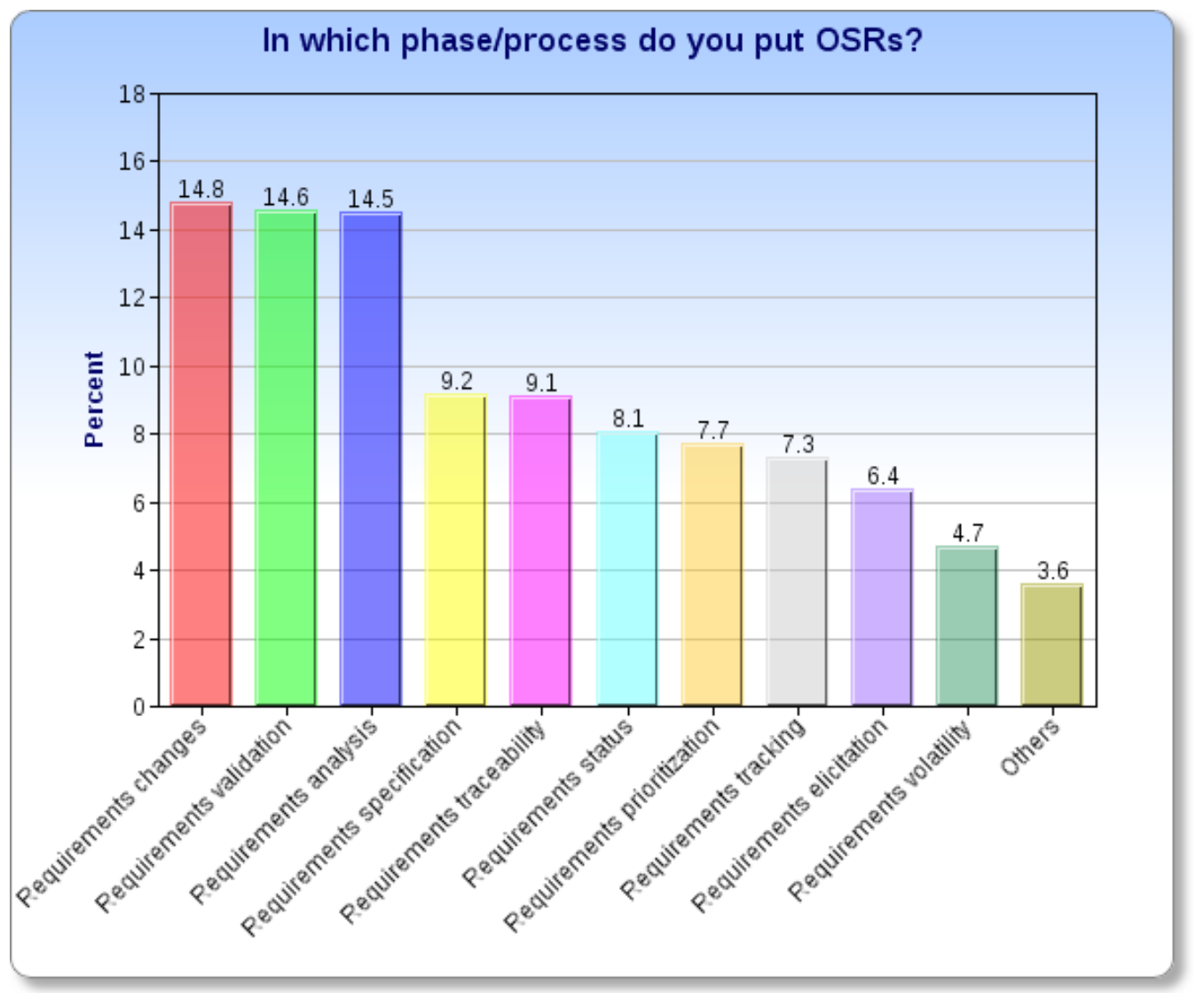

Figure 21: RE process and OSRs

For further analysis of the collected data, the author is looking for statistical association between the answers of this question (question 8 in Appendix A) on one side, and the respondents roles, and the development methodologies on the other side. During the analysis, the 'others' category of the phases of the requirements engineering variable and the development methodologies variable ware deleted, because many people wrote their comments about the question. These comments as mentioned earlier, were about not understanding the question. Thus, they are not relevant to the phases of the RE process.

Using chi-square test of significance, the null hypothesis $(\mathrm{HO})$ says that there is no significant association between the phases of the RE process and the respondents roles. The results of chi-square suggests that the null hypothesis (HO) is accepted $\left(\mathrm{X}^{2}=\right.$ $98.425, \mathrm{df}=80, \mathrm{p}=0.079>0.05)$. In addition, more than $20 \%$ of cells in this subtable have expected cell counts less than 5 . As a result, chi-square results may be invalid.

In the same way, the author hypothesizes (H1) that there is a significant relationship between the development methodology and the phases of the RE process. However, the chi-square test results are invalid due to violations to its conditions. These conditions are: more than $20 \%$ of cells have expected cell counts less than 5 , and some cells contain counts less than one. Therefore, H1 is rejected, this means that there is no significant relationship between the two variables.

In conclusion, the respondents have chosen all phases of RE life-cycle and requirements management in different degrees and preferred requirements changes, analysis, and validation. Henceforth, OSRs should be a part of all the phases, and should receive more attention in the requirements changes, analysis and validation phases. 


\section{Conclusion}

Requirements changes are unavoidable in software projects. Requirements change over time as software projects progress, and involved stakeholders (mainly customers) and developers gain better understanding of the final product. Additionally, time and budget constraints prevent implementing all candidate requirements and force project management to select a subset of requirements that are prioritized more important than the others so as to be implemented. As a result, some requirements become cancelled and deleted during the elicitation and specification phase while other requirements are considered not important during the prioritization phase. A common scenario in this situation is to leave the excluded requirements for being considered in the next release. The constant leaving of the excluded requirements for the next release may simply render them obsolete.

The focus of this master thesis is on obsolete software requirements (OSRs). The concept of OSRs seemed not to have any sensible interest in the research area. Therefore, there was no definition in the literature of what OSRs are, or how a requirement becomes obsolete, or how to manage them, or when to check for OSRs. Additionally, it was not known whether OSRs present any challenges, or have negative effects on software projects.

The major goal of this master thesis is to find empirical evidence concerning OSRs. Evidence such as the existence of OSRs in reality, the effects of OSRs on software projects, and the processes to discover OSRs and to take possible actions against them when they are discovered. Moreover, this thesis aims to define OSRs, to develop a classification for requirements that can help finding OSRs, and to inject the phenomenon of OSRs in the process of requirements engineering.

The outcome of this master thesis confirms that the phenomenon of OSRs does exist in reality. According to the results of the questionnaire, it is considered to be somehow serious by about $45 \%$ of responses, and serious by about $40 \%$ of responses. This master thesis has investigated the phenomenon through systematic literature review which showed that there is a paucity of information concerning this topic in the available literature. Furthermore, the web questionnaire was distributed in order to obtain answers to the research questions of the thesis. The results of the questionnaire were obtained through descriptive analysis and chi-square test of significance.

Although the author found some attempts to define concepts related to OSRs such as "obsolete functionality", and "dead features", he suggested an improved definition of OSRs based on the questionnaire results. Accordingly, an obsolete software requirement is a software requirement (implemented or not) that is no longer required for the current release or future releases, and it has no value (business goals) for the potential customers or users of a software product for various reasons e.g. (technological advancements).

Another contribution of the thesis is proposing processes or ways of detecting or finding OSRs, as well as suggesting possible actions to be taken against them when they are discovered. Nevertheless, majority of the questionnaire's respondents (54\%) suggest finding OSRs by reading the requirements manually, and after identifying the OSRs mark them as obsolete. Similarly, more than half of the questionnaire's responses agree on keeping OSRs in the documentation, and on providing the rationale why they became obsolete.

Additionally, OSRs are found to have effects on software projects depending on their sizes and types. To illustrate, the project size is represented by the number of requirements. The larger the project, the bigger the effects of OSRs on it are. Also, OSRs affect projects types. The respondents of the questionnaire, however, believe 
that outsourced projects are the most affected type followed by market-driven and bespoke projects, respectively.

Furthermore, the respondents of the questionnaire believe that OSRs should be taken care of during the whole requirements engineering life-cycle. However, the majority of respondents voted that requirements change $(14.8 \%)$, requirements validation (14.6\%), and requirements analysis (14.5\%) phases are the most important phases whereby one should look for OSRs. As well, OSRs should be recognized in the processes of requirements engineering in order to reduce their potential negative effects, especially, on large and very-large scale projects, and on outsourced projects.

The author developed a classification of software requirements based on their volatility. The classification could be used to find/detect/discover which types of requirement would be possibly rendered obsolete first. According to the results of the questionnaire, incorrect requirements, inconsistent requirements, and ambiguous requirements are the most three types of requirements to become obsolete first with scores of 3.88, 3.74, and 3.72, respectively. These types of requirements are known to be common problems in requirements, in general, thus, requirements specialists ought to take extra care of such types.

Moreover, there is a gap between academicians and researchers on one side, and software engineers and requirements specialists on the other side. Even though both parties consider OSRs a serious matter, only one researcher/academician recognizes OSRs in his/her requirements engineering process $(0.5 \%)$. As well, the lack in literature of topics related to change management, requirements volatility, and OSRs could be ascribed to this gap between researchers, and software practitioners. 


\section{Limitations}

This master thesis answers the research questions in Table 1 in page 9. This section discusses the limitations of the results of this master thesis. It is important here to remind the reader that all the research questions were answered through the web questionnaire (see section 2.2) but question number 2. Question number 2 was partially answered by both the SLR and by the questionnaire.

The limitations of this master thesis are based on the recommendations of [22], [60].

In general, questionnaires suffer common problems namely, sampling bias, and phrasing of questions so as to make everyone understand them. Control of sampling bias is considered one of the problems of surveys (questionnaire) because it affects the generalization (external validity) of the results [22]. In the case of this questionnaire, the author targeted software engineers, and requirements specialists, in general, as shown in section 2.2.3. By having majority of the respondents (representatives of the target population) coming from fields related to software engineering and requirements engineering, the results can be generalized.

Moreover, survey research might be affected by the way the questions are phrased. As a result, it is not guaranteed that all participants will understand them in the same way (this is called construct validity). In order to reduce the potential of this problem, the questionnaire was revised by the supervisors of this master thesis. As well, a pilot questionnaire was conducted on master students in software engineering. The feedback of the supervisors and the students was taken into consideration in improving the questionnaire (see Appendix A).

Furthermore, the author has detailed the steps that were taken during the SLR and the web questionnaire in order to ensure the reliability of the results of this master thesis. These detailed steps allow other researchers to replicate the study and obtain approximately the same results, especially, in the case of the SLR.

Additionally, the following paragraphs address the limitations of some research questions of this master thesis (internal validity).

Research question 1: does the phenomenon of obsolete requirements exist?

This question is answered in section 3. The answers of this question are partially found in the literature. There are few articles that talked about OSRs. However, in the questionnaire, the author assumed the existence of OSRs and instead asked the participants about the seriousness of OSRs. The results of the questionnaire suggest that OSRs exist and have different degrees of seriousness.

The limitations of research question 1 could be that some participants did not understand the meaning of the word "obsolete". In addition to, some authors refer to OSRs as "dead feature".

Research question 2: how can an obsolete requirement be defined?

This question is answered in section 3.2. The suggested definition of OSRs is based on what was found in the literature about OSRs, and the results of the questionnaire. Even though the questionnaire allows the participants to write their own definition of OSRs, the predefined definitions of OSRs might have influenced the opinions of the respondents.

Research question 3: what are the types of requirements that are eligible to become obsolete?

This question is answered in section 4. Section 4. presents a classification of requirements that was developed by the author. The classification was a hybrid of two classifications on volatile requirements. The developed classifications proposes types of software requirements. These types are not verified empirically. Moreover, the respondents of the questionnaire were asked to rate how likely the types of 
requirements will become obsolete. Here there are two possibilities, the first one is that the respondents might have not understood all the types of requirements. The second possibility is that some respondents might have chosen the rate randomly. Therefore, the results of this question should be taken with extra care.

Research question 4: When obsolete software requirements are defined/ identified/discovered, what is/are the possible action(s) to take against them (e.g. discard them, keep them)?

This question is answered in section 5. The questionnaire's respondents had to choose from a list of actions. They were allowed to choose more than one action, as well as writing down their own actions/suggestions.

The limitation of this question is that the proposed choices (i.e. actions) of this question might have influenced the opinions of the respondents.

Research question 5: what context factors, such as project size or domain/context that the project is running, does obsolete requirements affect?

This question is answered in section 6. The respondents of the questionnaire were asked to rate the effects of OSRs on the project's type and size. Additionally, this thesis only studies two dimensions of software projects.

The first dimension is the size of the project in terms of number of requirements. It is possible that some respondents use different metrics for the size of software projects such as Function Points [61]. Moreover, not all participants are expected to work on all sizes of projects.

The second dimension is the type of the project in terms of the business model (e.g. market-driven projects). This question could possibly have two limitations. The first limitation could be that some participants did not grasp the differences between the projects types even though a brief explanation was given. The second limitation is that some participants might not have experience in all types of projects and their answers were based on their expectations.

Note that other dimensions can be studied in the future such as the development methodology used.

Research question 6: where does obsolete requirements exactly put in the requirements engineering life cycle? e.g. in requirements traceability.

This question is answered in section 7. The participants of the questionnaire were asked to choose which requirements engineering phases are the most suitable ones to find OSRs in. The results of this question showed a major problem. As reported in section 7., 11 people out of 217 did not understand the meaning of the word "put" in the context of the question. Furthermore, it is not guaranteed that all respondents are familiar or aware of the steps of each phase (because only the name of the phase was mentioned). Therefore, the results of this question should be treated with extra care. 


\section{Future Work}

This master thesis investigates the phenomenon of OSRs. The outcome of this master thesis is preliminary and further work is required in order to cover more concepts related to OSRs. As well, more research has to be done in order to overcome the limitations of this master thesis (see section 9.).

Since there is a relationship between OSRs and types of requirements. Some types of requirements become obsolete before others as shown in section 4.1, more empirical work is required in order to find the relationships between OSRs and different types of requirements. Moreover, in this thesis, the author connected obsolescence of software requirements to requirements volatility. Requirements volatility is relatively a new topic and small amount of researches have been conducted on it so far. Thus, more efforts and empirical researches are needed to find the relationships between volatile requirements and OSRs.

On the other hand, many requirements are dependent on each others. In case a requirement becomes obsolete, it will definitely influence other requirements in the system. Thus, requirements dependency and requirements traceability are important topics that should be studied in relations to OSRs. The effects of OSRs go beyond the requirements documentation to the architecture of the system, the code, the tests, and the maintenance of the system. Furthermore, requirements become obsolete at any time. Some of them become obsolete early in the project before they are implemented whereas other requirements become obsolete after the implementation of the system. More research such as [26] have to be done in order to reduce the effects of OSRs late in the projects.

OSRs could have effects on software projects from several dimensions other than the size and the type of the projects which this thesis have discussed. Consequently, more work is required to empirically examine the effects of OSRs on other dimensions such as the performance of the software projects, the defects density, and the available resources of the project.

Additionally, in section 5.2, the author suggested several processes to discover OSRs, and proposed ways of treating OSRs after they are discovered. These processes are not validated in the industry nor in the academia. So, improvement of these processes, and may be suggesting more processes could be done in the future. As well, validation of the suggested OSRs processes from requirements experts is required.

On the other hand, OSRs processes should be integrated in the requirements engineering life-cycle. This integration has to be supported by requirements management tools. Thus, some work is required to include some of the suggested OSRs processes into requirements management tools. This could be done using an open source requirements management tools, or creating a requirements management tool from scratch that takes into consideration OSRs.

Additionally, it is necessary to find out the advantages of finding OSRs in a project from different perspectives such as the defects density. 


\section{References}

[1] G. Kotonya and I. Sommerville, Requirements Engineering: Processes and Techniques. Wiley, 1998, p. 294.

[2] C. Hood, S. Wiedemann, S. Fichtinger, and U. Pautz, Requirements Management: The Interface Between Requirements Development and All Other Systems Engineering Processes. Springer, 2010, p. 275.

[3] A. Aurum and C. Wohlin, "The fundamental nature of requirements engineering activities as a decision-making process," Information and Software Technology, vol. 45, no. 14, pp. 945-954, Nov. 2003.

[4] B. Regnell, R. Svensson, and K. Wnuk, "Can We Beat the Complexity of Very LargeScale Requirements Engineering?," vol. 5025, B. Paech and C. Rolland, Eds. Berlin, Heidelberg: Springer Berlin Heidelberg, 2008, pp. 123-128.

[5] C. Jones, Applied software measurement: global analysis of productivity and quality, Third. McGraw-Hill Professional, 2008, p. 662.

[6] S. Group, “T23E-T10E STANDISH GROUP REPORT," 1995. [Online]. Available: http://www.spinroot.com/spin/Doc/course/Standish_Survey.htm. [Accessed: 24-Mar2011].

[7] A. Loconsole, "Definition and validation of requirements management measures," Umeå University, 2007.

[8] D. Zowghi and N. Nurmuliani, "A study of the impact of requirements volatility on software project performance," in Ninth Asia-Pacific Software Engineering Conference, 2002., 2002, pp. 3-11.

[9] C. Wohlin and A. Aurum, "What is important when deciding to include a software requirement in a project or release?," in 2005 International Symposium on Empirical Software Engineering, 2005., 2005, pp. 237-246.

[10] Y. K. Malaiya and J. Denton, "Requirements volatility and defect density," in Proceedings 10th International Symposium on Software Reliability Engineering (Cat. No.PR00443), 1999, pp. 285-294.

[11] G. E. Stark, P. Oman, A. Skillicorn, and A. Ameele, "An examination of the effects of requirements changes on software maintenance releases," Journal of Software Maintenance: Research and Practice, vol. 11, no. 5, pp. 293-309, Sep. 1999.

[12] T. Gorschek and M. Svahnberg, "Requirements Experience in Practice: Studies of Six Companies," in Engineering and Managing Software Requirements, A. Aurum and C. Wohlin, Eds. Springer Verlag, 2005, pp. 405-426.

[13] J. Natt och Dag and V. Gervasi, "Managing Large Repositories of Natural Language Requirements," in Engineering and Managing Software Requirements, A. Aurum and C. Wohlin, Eds. Berlin/Heidelberg: Springer-Verlag, 2005, pp. 219-244-244.

[14] D. Murphy and D. Rooney, "Investing in Agile: Aligning Agile Initiatives with Enterprise Goals," Cutter IT Journal, vol. 19, no. 2, pp. 6 - 13, 2006.

[15] T. C. Lethbridge, J. Singer, and A. Forward, "How software engineers use documentation: The state of the practice," IEEE Software, vol. 20, no. 6, pp. 35-39, Nov. 2003 
[16] T. Hammer, L. Huffman, L. H. Rosenberg, W. Wilson, and L. E. Hyatt, "Doing Requirements Right the First Time," CROSSTALK: Journal of Defense Software Engineering, p. 22, 1998.

[17] "Cambridge Dictionaries Online, Entry: obsolete," 2011. [Online]. Available: http://dictionary.cambridge.org/dictionary/british/obsolete. [Accessed: 01-Mar-2011].

[18] J. Savolainen, I. Oliver, and M. Mannion, "Transitioning from Product Line Requirements to Product Line Architecture," in 29th Annual International Computer Software and Applications Conference (COMPSAC'05), 2005, pp. 186-195.

[19] M. Mannion, O. Lewis, H. Kaindl, G. Montroni, and J. Wheadon, "Representing Requirements on Generic Software in an Application Family Model," in Software Reuse: Advances in Software Reusability, vol. 1844, W. B. Frakes, Ed. Berlin, Heidelberg: Springer Berlin Heidelberg, 2000, pp. 205-211-211.

[20] N. Nurmuliani, D. Zowghi, and S. Fowell, "Analysis of requirements volatility during software development life cycle," in 2004 Australian Software Engineering Conference. Proceedings., 2004, pp. 28-37.

[21] L. Merola, "The COTS Software Obsolescence Threat," in Fifth International Conference on Commercial-off-the-Shelf (COTS)-Based Software Systems (ICCBSS'05), pp. 127-133.

[22] S. Easterbrook, J. Singer, M.-A. Storey, and D. Damian, "Selecting Empirical Methods for Software Engineering Research," in Guide to Advanced Empirical Software Engineering, F. Shull, J. Singer, and D. I. K. Sjøberg, Eds. London: Springer London, 2008, pp. 285-311-311.

[23] B. Kitchenham and S. Charters, "Guidelines for performing Systematic Literature Reviews in Software Engineering," Engineering, vol. 2, no. EBSE 2007-001, 2007.

[24] C. W. Dawson, Projects in Computing and Information Systems: A Student's Guide. Addison-Wesley Professional, 2009, p. 304.

[25] M. Unterkalmsteiner, T. Gorschek, A. K. M. M. Islam, C. K. Cheng, R. B. Permadi, and R. Feldt, "Evaluation and Measurement of Software Process Improvement - A Systematic Literature Review," IEEE Transactions on Software Engineering, Mar. 2011.

[26] E. B. Charrada, "Updating requirements from tests during maintenance and evolution," in Proceedings of the eighteenth ACM SIGSOFT international symposium on Foundations of software engineering - FSE '10, 2010, p. 337.

[27] F. Loesch and E. Ploedereder, "Restructuring Variability in Software Product Lines using Concept Analysis of Product Configurations," in 11th European Conference on Software Maintenance and Reengineering (CSMR'07), 2007, pp. 159-170.

[28] D. Muxworthy, "Language architecture - obsolescence and deletions," Computer Standards \& Interfaces, vol. 18, no. 4, pp. 303-307, Aug. 1996.

[29] M. Maram, P. Prabhakaran, S. Murthy, and N. Domala, "Sixteen Roles Performed by Software Engineers in First One Year," in 22nd Conference on Software Engineering Education and Training, 2009, pp. 212-215.

[30] C. Laporte, M. Doucet, P. Bourque, and Y. Belkébir, "Utilization of a Set of Software Engineering Roles for a Multinational Organization," in Product-Focused Software Process Improvement, vol. 4589, J. Münch and P. Abrahamsson, Eds. Berlin, Heidelberg: Springer Berlin Heidelberg, 2007, pp. 35-50-50. 
[31] R. L. Glass and I. Vessey, "Contemporary application-domain taxonomies," IEEE Software, vol. 12, no. 4, pp. 63-76, Jul. 1995.

[32] M. A. Awad, "A Comparison between Agile and Traditional Software Development Methodologies," The University of Western Australia, 2005.

[33] "SELECTING A DEVELOPMENT APPROACH," 2008. [Online]. Available: http://www.cms.hhs.gov/SystemLifecycleFramework/Downloads/SelectingDevelopm entApproach.pdf. [Accessed: 15-Apr-2011].

[34] O. Gotel and P. Mader, "How to Select a Requirements Management Tool: Initial Steps," in 17th IEEE International Requirements Engineering Conference, 2009, pp. 365-367.

[35] L. M. Rea and R. A. Parker, Designing and Conducting Survey Research: A Comprehensive Guide, Third. San Francisco: Jossey-Bass, 2005, p. 304.

[36] SPSS, “Online Help of SPSS version 17.” IBM, 2008.

[37] "definition of obsolete from Oxford Dictionaries Online." [Online]. Available: $\mathrm{http} / / /$ oxforddictionaries.com/view/entry/m_en_gb0572060\#m_en_gb0572060. [Accessed: 01-Mar-2011].

[38] "Definition of obsolete adjective from Cambridge Dictionary Online: Free English Dictionary and Thesaurus." [Online]. Available:

http://dictionary.cambridge.org/dictionary/british/obsolete. [Accessed: 01-Mar-2011].

[39] "Free Online Thesaurus of Synonyms and Antonyms, Entry: obsolete." [Online]. Available: http://thesaurus.com/browse/obsolete. [Accessed: 01-Mar-2011].

[40] "Definition of deprecate verb (NOT VALUE) from Cambridge Dictionary Online: Free English Dictionary and Thesaurus." [Online]. Available: http:/dictionary.cambridge.org/dictionary/british/deprecate_2\#deprecate_2_3. [Accessed: 01-Mar-2011].

[41] IEEE Recommended Practice for Software Requirements Specifications (830-1998). New York, New York, USA: , 1998, p. vi+31.

[42] C. Development, "CMMI® for Development, Version 1.3 CMMI-DEV, V1.3," Engineering, no. November, 2010.

[43] P. Bourque and R. Dupuis, Guide to the Software Engineering Body of Knowledge 2004 Version. Angela Burgess, 2004.

[44] D. Leffingwell and D. Widrig, Managing Software Requirements: A Use Case Approach (2nd Edition), 2nd ed. Addison-Wesley Professional, 2003, p. 544.

[45] J. Stephen, J. Page, J. Myers, A. Brown, D. Watson, and I. Magee, SYSTEM ERROR Fixing the flaws in government IT. London: , 2011, p. 100.

[46] T. Herald, D. Verma, C. Lubert, and R. Cloutier, "An obsolescence management framework for system baseline evolution-Perspectives through the system life cycle," Systems Engineering, vol. 12, no. 1, pp. 1-20, Dec. 2009.

[47] A. Aurum and C. Wohlin, "Requirements Engineering: Setting the Context ," in Engineering and Managing Software Requirements, A. Aurum and C. Wohlin, Eds. Berlin/Heidelberg: Springer-Verlag, 2005, pp. 1-15-15.

[48] X. Shan, G. Jiang, and T. Huang, "The Study on Knowledge Transfer of Software Project Requirements," in International Conference on Biomedical Engineering and Computer Science, 2010, pp. 1-4. 
[49] S. D. P. Harker, K. D. Eason, and J. E. Dobson, "The change and evolution of requirements as a challenge to the practice of software engineering," in Proceedings of the IEEE International Symposium on Requirements Engineering, 1993, pp. 266272.

[50] S. McGee and D. Greer, "A Software Requirements Change Source Taxonomy," in Fourth International Conference on Software Engineering Advances, 2009, pp. 5158.

[51] J. Kabbedijk, K. Wnuk, B. Regnell, and S. Brinkkemper, "What decision characteristics influence decision making in market-driven large-scale software product line development?," Hildesheimer Informatik-Berichte, vol. 2010, pp. 42-53, 2010.

[52] K. Wnuk, B. Regnell, and L. Karlsson, "What Happened to Our Features? Visualization and Understanding of Scope Change Dynamics in a Large-Scale Industrial Setting," in 2009 17th IEEE International Requirements Engineering Conference, 2009, pp. 89-98.

[53] "Manifesto for Agile Software Development." [Online]. Available: http://agilemanifesto.org/. [Accessed: 14-Aug-2011].

[54] B. Regnell and S. Brinkkemper, "Market-Driven Requirements Engineering for Software Products," in Engineering and Managing Software Requirements, A. Aurum and C. Wohlin, Eds. Berlin/Heidelberg: Springer-Verlag, 2005, pp. 287-308308.

[55] K. E. Wiegers, Software requirements: practical techniques for gathering and managing requirements throughout the product development cycle, 2nd ed. Microsoft Press, 2003, p. 516.

[56] I. Sommerville and P. Sawyer, Requirements engineering: a good practice guide. John Wiley \& Sons, 1997, p. 391.

[57] E. Hull, K. Jackson, and J. Dick, Requirements Engineering, 3rd ed. Springer, 2010, p. 207.

[58] J. M. Bhat, M. Gupta, and S. N. Murthy, "Overcoming Requirements Engineering Challenges: Lessons from Offshore Outsourcing," IEEE Software, vol. 23, no. 5, pp. 38-44, Sep. 2006.

[59] R. Awan, "Requirements Engineering Process Maturity Model for Market Driven Projects," Blekinge Tekniska Högskola, 2005.

[60] R. K. Yin, Case Study Research: Design and Methods, Third Edition, Applied Social Research Methods Series, Vol 5, Third. Sage Publications, Inc, 2002, p. 200.

[61] "Function Points, Measures, and Software Metrics." [Online]. Available: http://www.functionpoints.com/. [Accessed: 14-Aug-2011]. 


\section{Appendix A: Obsolete Software Requirements QuestionnaIre}

Software requirements keep changing over the course of a project. The changes can be due to better understanding of the final product by stakeholders, technological advancements, and other factors. Furthermore, the continuous changes leave many requirements unimplemented or forgotten in the documentation. Therefore, over time, many requirements become out-of-date/outdated/obsolete and can cause information overload for the stakeholders. The aim of this questionnaire is to investigate the phenomenon obsolete software requirements, if it exists, how it is handled and impacts the development work.

The survey should take about 5-7 minutes of your time. Please answer how YOU perceive the situation to be today, NOT how it should or could be.

DISCLAIMER: all the information will be kept confidential and no traceability to individuals will ever be possible.

1. How do YOU define obsolete software requirement? (choose one or several) [multiple choice]

It is a software requirement that...

a) is no longer required for the current release for various reasons.

b) has no value for the potential users in the current release.

c) is duplicated/redundant in the current release.

d) is rejected/refused to be included in the current release for various reasons.

e) was never used or implemented in the product.

f) Others (please specify)

2. To what extent do you think the phenomenon of obsolete software requirements is serious? (choose one) [single choice]
a) Trivial
b) Somehow serious
c) Serious
d) Very serious

3. How likely the following types of software requirements will become obsolete.

On a scale from 1 to 5,1 is not likely and 5 is very likely. Please tick $(\sqrt{ })$

$1=$ Not likely, $2=$ somehow likely, $3=$ likely, $4=$ more likely, $5=$ very likely

\begin{tabular}{|l|l|l|l|l|l|}
\hline \multicolumn{1}{|c|}{ Software Requirements Types } & 1 & 2 & 3 & 4 & 5 \\
\hline Requirements related to laws and regulations. & & & & & \\
\hline Requirements related to standards. & & & & & \\
\hline $\begin{array}{l}\text { Requirements obtained from market analysis, } \\
\text { domain experts and competitors. }\end{array}$ & & & & & \\
\hline $\begin{array}{l}\text { Requirements about the company's organization } \\
\text { and policies. }\end{array}$ & & & & & \\
\hline Hardware related requirements. & & & & & \\
\hline $\begin{array}{l}\text { Functional requirements originated from } \\
\text { customers. }\end{array}$ & & & & & \\
\hline $\begin{array}{l}\text { Functional requirements originated from end } \\
\text { users. }\end{array}$ & & & & & \\
\hline
\end{tabular}




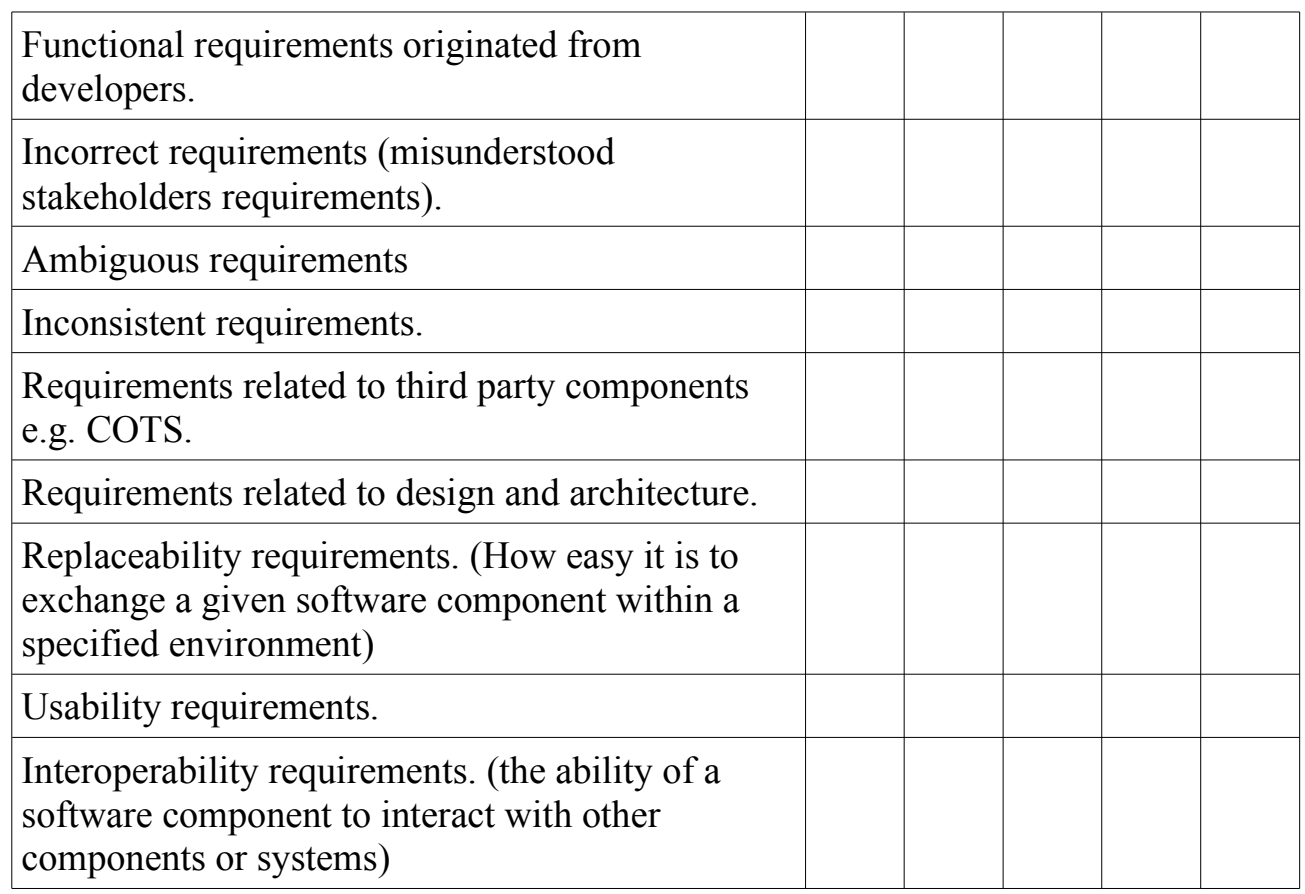

4. How do you discover/find/detect obsolete software requirements in a software requirement document or in a software requirements database? (choose one or several) [multiple choice]

a) I never found them / I never thought of finding them.

b) Manually, by reading the requirements specification and marking requirements as obsolete.

c) Automatic, using built in search functionalities that look for certain attributes.

d) I have a predefined status called obsolete.

e) Others (please specify)

5. In your opinion, what is/are the appropriate action(s) to be taken against obsolete requirements in a software requirement database or in a software requirement specification (SRS) document? (choose one or several) [multiple choice]

a) Delete/remove them from the database/document.

b) Keep them, but assign them a status called "obsolete".

c) Keep them, but assign them a status called "obsolete" and write the reason why they become obsolete for future reference (database/document).

d) Move them into a separated section titled obsolete requirements or the like (mainly in documents).

e) Others (please specify)

6. How likely are obsolete requirements to affect/impact the size of the project. On a scale from 1 to 5,1 is not likely and 5 is very likely. Please tick $(\sqrt{ })$

$1=$ Not likely, $2=$ somehow likely, $3=$ likely, $4=$ more likely, $5=$ very likely

\begin{tabular}{|l|l|l|l|l|l|}
\hline \multicolumn{1}{|c|}{ Software Project Size } & 1 & 2 & 3 & 4 & 5 \\
\hline Small scale requirements (tens of requirements) & & & & & \\
\hline $\begin{array}{l}\text { Medium scale requirements (hundreds of } \\
\text { requirements) }\end{array}$ & & & & & \\
\hline Large scale requirements (thousands of & & & & & \\
\hline
\end{tabular}




\begin{tabular}{|l|l|l|l|l|l|}
\hline requirements) & & & & & \\
\hline $\begin{array}{l}\text { Very-large scale requirements (over ten thousand } \\
\text { requirements) }\end{array}$ & & & & & \\
\hline
\end{tabular}

7. How likely the type of project (context) will be affected by obsolete requirements. On a scale from 1 to 5,1 is not likely and 5 is very likely. Please tick $(\sqrt{ })$ $1=$ Not likely, $2=$ somehow likely, $3=$ likely, $4=$ more likely, $5=$ very likely

\begin{tabular}{|l|l|l|l|l|l|}
\hline \multicolumn{1}{|c|}{ Software Project Size } & 1 & 2 & 3 & 4 & 5 \\
\hline $\begin{array}{l}\text { Bespoke projects (software that is specially } \\
\text { developed for some specific organization). }\end{array}$ & & & & & \\
\hline $\begin{array}{l}\text { Market-Driven projects (software that is made for } \\
\text { the open market place). }\end{array}$ & & & & & \\
\hline $\begin{array}{l}\text { Outsourced projects (software that is given to a } \\
\text { third party to produce it). }\end{array}$ & & & & & \\
\hline
\end{tabular}

8. In the software requirements engineering life-cycle in general, and in the software requirements management in particular, in which phase/process do YOU put obsolete requirements? (choose one or several) [multiple choice]
a) Requirements elicitation.
b) Requirements analysis.
c) Requirements specification
d) Requirements validation.
e) Requirements changes.
f) Requirements status.
g) Requirements traceability.
h) Requirements tracking.
i) Requirements prioritization.
j) Requirements volatility.
k) Others (please specify)

9. Do you have your own process of requirements engineering that takes the phenomenon of obsolete requirements into account?

a) No.

b) If Yes please describe it?

\section{Demographic Questions:}

10. What is your main role in your organization? (choose one) [single choice]
a) Software project manager (responsible for the overall project perspective and planning of project evolution).
b) Software product manager (responsible for work mapping, release planning, scoping, and business aspects of a product).
c) Software team leader (responsible for managing and prioritizing requirements within the realization phases of a project).
d) Software architect/designer
e) Software requirements analyst/engineer/coordinator 
f) Developer/programmer/coder.

g) Software Integrator

h) Software tester/test designer

i) Support and maintenance analyst/software maintainer

j) Researcher/academician.

k) Others (please specify)

11. What type of business or domain do you work on? (choose one or several) [multiple choice]

a) Information technology/Computer and software services

b) Engineering (automotive, industrial, manufacturing, energy, etc.)

c) Telecommunications

d) Consultancy

e) Transportation

f) Construction

g) Banking/Finance

h) Health/medical

i) Education

j) Insurance / legal

k) Government / military

1) Public sector / administration

m) Entertainment

n) Media / news / publishing

o) Others (please specify)

12. What is the size of your organization? (choose one) [single choice]
a) Less than 10 employees.
b) $11-20$ employees.
c) $21-50$ employees
d) $51-100$ employees.
e) $101-200$ employees
f) $201-500$ employees
g) over 501 employees.

13. What is the average duration of typical projects you are involved in? (choose
one) [single choice]
a) 0 - 3 months
b) $4-6$ months.
c) $7-12$ months.
d) $13-24$ months.
e) $25-36$ months.
f) Over 37 months.

14. What is the development methodology/process do you usually follow? (choose one or several) [multiple choice]
a) Ad-hoc (no method).
b) Waterfall development.
c) Incremental/evolutionary development.
d) Spiral development.
e) Agile software development.
f) Rapid application development.
g) Prototyping.
h) Rational Unified model 
i) Components-based software engineering

j) Formal methods

k) Others, please specify

15. What type of requirements engineering have you been involved in? (choose one or several) [multiple choice]

a) Bespoke of contract driven requirements engineering (software that is specially developed for some specific organization).

b) Market-Driven requirements engineering (software that is made for the open market place).

c) Outsourced projects (software that is given to a third party to produce it).

d) Open Source projects.

e) Others (please specify)

Mapping of research questions (Table 1 in page 9), and the questions in the questionnaire.

\begin{tabular}{|c|c|}
\hline Research Question & Questionnaire Question \\
\hline 1. Does the phenomenon of obsolete requirements exist? & $\mathrm{Q} 2$ \\
\hline 2. How can an obsolete requirement be defined? & $\mathrm{Q} 1$ \\
\hline 3. What are the types of requirements that are eligible to \\
become obsolete? \\
$\begin{array}{l}\text { 3.1. What is/are the possible process(es) to discover/find/ } \\
\text { expose/detect/mark obsolete requirements? }\end{array}$
\end{tabular}




\title{
Appendix B: Opposition Report 1
}

This appendix contains the first opposition report written by Ali Al-Refai. The opposition report's contents are included as received from Ali. However, I have changed the original format of his report in order to be consistent with this thesis report's format. As well, my comments are included wherever appropriate in Courier New font.

Note: The references mentioned in this appendix refer to the reference section at the end of this same appendix.

\section{Opposition report for Master Thesis: Obsolete Software Requirements (MSE-2011-60)}

Name: Ali Al-Refai, Swedish Personal Number: 820508-0552, Email: ala107@student.bth.se.

\begin{abstract}
This paper serves as the opposition report for the master thesis project presented by Showayb A. A. Zahda on Thursday, September 15, 2011. The aim of this paper is to address the critical and important findings on the thesis report, which can enhance the readability and quality of the report.
\end{abstract}

\section{INTRODUCTION}

In general, the master thesis entitled "Obsolete Software Requirements" is a welldone report. For most parts of the report, it is easy and clear to read and understand, however, at some few parts it could have been structured clearer. The thesis report consists of seven main sections that characterize the core work of the thesis, additional to those sections; Conclusion, Limitations, Future work, and References are also presented. The first section presents an introduction on requirement engineering in general and linking that to Obsolete Software Requirements (OSRs). The rest of this master thesis report is organized as follows: section 2 describes the research methodologies followed in this master thesis, and it presents some results of the questionnaire. While section 3 defines OSRs and examines its existence and seriousness, section 4 develops a classification of requirements based on the volatility requirements notion. As well, section 4 provides basic relationships between OSRs and types of requirements. In section 5, the author describes possible ways/processes to find or detect OSRs in the requirements documentation. Moreover, he suggests some actions to be taken against OSRs after they are discovered. Section 6 studies the effects of OSRs on the project's size, and on the project's type. In section 7, OSRs are connected to the whole process of requirements engineering and requirements management processes. Finally, the conclusion of the master thesis, its limitations, and future works are presented in section 8 , section 9 and section 10, respectively. This paper is aimed to report the critical and important findings for further improvement of the master thesis report. The remaining of this paper is organized as follow. Section 2 discusses the review of the master thesis report based on guidelines for opposition and the rubrics for assessing master thesis report [1]. Section 3 presents the conclusion of the review.

\section{REVIEW OF THESIS REPORT}

The following section discuses the review of the thesis report. 


\subsection{Research problem, aims and objectives}

The author of the thesis report presents the research problem of the study in section two. Research questions are clearly defined together with the aim of each question. The questions seems to be covering most of the aspects regarding to phenomenon of obsolete software requirements, however we think that the author did not draw enough attention to few other aspects (i.e. 1) What is the advantage(s) of discovering/identifying the OSRs in a project? 2) What is the impact(s) of OSRs on the software development process? 3) How to get the maximum benefits from OSRs to help the software development process?) (Indeed, these questions are interesting and could be considered for future work. Due to resources limitations, the scope of this master thesis was limited to the research questions presented in Table 1). On the other hand related work is not presented or discussed in this section. Published guidelines state the significance of simplifying how the work to be stated relates to existing previous work [2]. (That's true, however, there is no related work in the literature regarding OSRs, refer to section 3.3 for more information about OSRs in the literature.)

The author of the thesis report presents the research problem of the study in the Research Methodology section. The author provides brief information about the motivation of the work in the Introduction and Research Methodology sections. The methodology used to answer the research questions is also described in the same chapter. There are seven research questions in this thesis project. Considering the scope of the project and the research methodology presented the report, the research questions could have been presented in a more focused way (i.e. collecting related questions together as questions and sub questions) so it will help the readability of the problem. On the other hand, designing a diagram to link the research questions to the research methods in a visual way could have been an excellent tool to assist the readers of the report (Appendix A contains a table that maps the questions in the questionnaire with the research question).

\subsection{Research methods}

As described in the report, the thesis project is conducted by using two research methods (literature review and Survey - Web Questionnaire). The SLR is following the recommendation of Kitchenham [2] and is inspired by another SLR done by Unterkalmsteiner et al. [3]. The reason of conducting this method was described in the abstract as "to find relevant information about OSRs" but it was not describing what kind of information or how relevant they are! (More information can be found in section 2.1) This approach is aligned with the aims and objectives of the thesis. However, the authors do not provide clear information about how each step is performed and what the result of each step is (Section 2.1 clearly state the outcome of the SLR). The author does not provide convince reasoning to motivate the use of such methods (i.e. "The reason behind using [25] is that it follows Kitchenham's guidelines [23], and it presents a good and recent example of conducting SLR." This is not an enough motivation!) (The reason to use [25] is to complement Kitchenham's guidelines. Moreover, I used [25] because it includes diagrams that show the sequence of steps to conduct a SLR and how to present their results in a good way. Now, I explicitly stated that [25] complements Kitchenham's guidelines). As for the questionnaire, again the ambiguity and readability issues were arise, since it was difficult to keep following as a reader without clear assisting diagrams that demonstrate the flow of the work i.e. "Moreover, the results of the SLR (presented in section 3.3.1) contributions to the questionnaire were really subtle 
because they did not produce any solid evidence about OSRs. Therefore, the author had to suggest the questions and possible answers to them." (The paragraph has been rephrased to clearly state that the SLR outcome was not enough to formulate the questions in the questionnaire). However the motivation of using the statistical methods for analyzing the questionnaire (i.e. Likert Scale, Chi-square and Cramer's V) should be clearly stated (The usage of any statistical method depends on the date and their types i.e. nominal data, ordinal ect. Therefore, these methods were used.).

\subsection{Research results}

The results presented in the thesis report are generally good. Some of the results need to be thought about. Figure 13 is stating "Incorrect Requirement" as a requirements type with a highest likelihood to become obsolete. Why "Incorrect Requirements" should be considered from the first place? (Section 4 presents types of requirements and their likelihood to become obsolete. Incorrect requirements is one type of requirements that has been identified as a source of change in [50]. As well, there is no agreed-on classification of requirements. Thus, it is possible that other researchers do not agree that incorrect requirements can be a type of requirements.)

\subsection{Knowledge of the research area}

The author has demonstrated good knowledge covering the research area. However, the knowledge gained from literature review is not used in optimum way (i.e. defining OSRs) (Indeed, the definition of OSRS was inspired from the finding of the literature. I explicitly mentioned in section 3.2 "On the other hand, the choices were either inspired from the few definitions found in the literature (see section 3.3) or suggested by the author."). It was clear that there is a lack of literatures in the research phenomenon however it would gave more sense if the results gained from the questionnaire was utilized to formulate the search strings and not to rely only on the literature meaning of the word Obsolete. (The SLR was executed first in order to find possible answers for the research questions, and then I conducted the web questionnaire. This means it is not possible to use the results of the questionnaire in forming the search string of the SLR.)

\subsection{Discussion}

Overall, the discussions in this thesis report are enough contribution. The authors do not discuss deeper on the findings of literature review nor linking the SLR findings with respondents' answers and the author personal thoughts. More intensive literature review on this topic will improve the quality of discussion. The authors should focus on the important threats that give major impact to the result of the study. The preventive actions to reduce the threat also introduce a new threat. For example, involving only one researcher to review the Study Quality Assessment might lead to bias the study since it is very subjective. Other example is the use of statistical test in the questionnaire results since certain tests have assumptions on, i.e. independent samples, etc. (This paragraph is very vague and broad. Additionally, it shows that the opponent is writing falsely statements especially when saying that "involving only one researcher to review the Study Quality Assessment". In fact, there was not Study Quality Assessment in the SLR as stated in section 2.1.5 because the SLR did not yield sufficient results to asses their quality.) 


\subsection{Conclusion}

The conclusions of the thesis project are well presented. However, the author does not consistent with the way to draw all conclusions. Rather than based on empirical data, the author draw conclusion based on what he believe (Again this is a falsely claim, the conclusions are drawn based on the finding of the questionnaire and the SLR). There is no strong argument when the author claims in section 3 "this thesis presents the definition of OSRs based on the experience of the author and the results of the questionnaire."

\subsection{Reference list and the use of sources/references}

There are some serious errors in the formatting of the reference list. The author does not follow the proper reference formatting for many of the research articles and web recourses (i.e. References no. 6, 17, 33, 37, 38, 39, 40, 53, and 61). There should be a proper referencing according to the master thesis format. (This thesis follows the IEEE citation standard. Corrections were made where applicable.)

\subsection{Language, structure and formatting}

In general, the format of the thesis is good enough, however the author should consider more attention to the structure of the thesis so it help the readability of the work (i.e. expressing the chapters in smaller sections and sub-sections). It starts with proper background and motivation of the research. In general the language of the thesis is plain with few grammatical and spilling mistakes.

\section{CONCLUSION}

Overall, the thesis report is well-written, the topic is unique and presenting some genuine results. Critical reviews have been presented based on the report and the presentation of the thesis. It seems that the author have followed the research methodology presented in the report but might have not structured the thesis in an "easy-follow" way. There are also numbers of errors regarding the formatting of the reference list; they should be referenced according to the master thesis format.

\section{REFERENCES}

[1] Guidelines for opposition, version 1.4, 2011-06-09, T. Gorschek \& R. Feldt, [Online].

Available: http://iaser.tek.bth.se/pa2403/guidelines_for opposition.pdf

[2] B. Kitchenham and S. Charters, "Guidelines for performing Systematic Literature Reviews in Software Engineering," Engineering, vol. 2, 2007.

[3] M. Unterkalmsteiner, T. Gorschek, A.K.M.M. Islam, C.K. Cheng, R.B. Permadi, and R.Feldt, "Evaluation and Measurement of Software Process Improvement - A Systematic Literature Review," IEEE Transactions on Software Engineering, Mar.2011. 


\section{ApPendix C: Opposition Report 2}

This appendix contains the second opposition report written by Ramya Chowdary Veeramachaneni. The opposition report's contents are included as received from Ramya. However, I have changed the original format of her report in order to be consistent with this thesis report's format. As well, my comments are included wherever appropriate in Courier New font.

\section{Master thesis opponent report}

Opponent: Ramya Chowdary Veeramachaneni, 8804212127, rave10@student.bth.se

Thesis Title: Obsolete Software Requirements

Thesis Number: MSE-2011-60

Author: Showayb A.A. Zahda

\section{Major Comments:}

\section{Chapter 1: Introduction}

Page 3: In introduction, you have mentioned the statement "However, a large and very large volume of requirements require a systematic approach supported by requirements management tools." Are there any explicit tools for requirements management process? If so, mention them. (The list of requirements management tool is very long and I do not find it necessary to name any.)

Page 3: "A common scenario in this situation is to leave the excluded requirements for being considered in the next release." Is this statement applicable for be-spoke, market-driven and outsourced projects? (Yes)

Page 4: "The main objectives of this master thesis are: to define what OSRs are, to find the relationships between OSRs and requirements types, to propose processes to discover OSRs and to take possible actions against them when they are discovered, and to study the effects of OSRs." Stating these objectives with bullets will make reader understand the text better.

Page 5: It would be better if you would write the organization of thesis report as a separate sub-section using bullets like say

- Section 2: describes the research methodology....

- Section 3: defines OSRs.

$$
\text { (The suggestion was considered) }
$$

\section{Chapter 2: Research methodology}

Page 6: "However, Software Engineering research can be conducted using several research methodologies namely: controlled experiments, case studies (both exploratory and confirmatory), survey research, ethnography, and action research [22]." What about interviews? I think interview is also a research methodology used in software engineering research context. (Interviews are not suitable in this case because we did not have sufficient knowledge about OSRs. As well, interviews are slower, limited to less audience, consume more resources, and would yield less accurate results in comparison with the web questionnaire)

Page 6: "As well, the use of the questionnaire is to complement the outcome of the SLR and strengthen the results." This sentence seems to be conflicting with the above mentioned statement "However, the outcome of the SLR was not helpful in answering the research questions of this master thesis concerning OSRs." (The sentence is rephrased to: As well, the use of the questionnaire is to 
complement the outcome of the SLR in answering the research questions of this master thesis.)

Page 6: "The SLR in this thesis follows the recommendations of Kitchenham [23] and is inspired by another SLR done by Unterkalmsteiner et al. [25]." Change the grammar mistake. (Corrected)

Page 6: "The reason behind using Unterkalmsteiner et al. [25] is that it follows Kitchenham's guidelines [23], and it presents a good and recent example of conducting SLR." Insert the author name as the sentence seems to be incomplete. (No changes, when there are several authors of a paper, the standard is to mention the name of the first author followed by et al.)

Page 7: Kitchenham suggests PICO criteria for formulating search string. Which criteria did you use for formulating search string? (See section 2.1.1 for more information about how the search string was formulated)

When you have got only 3 partial relevant papers in SLR, why did you not prefer literature review? You could have done snow ball sampling so that you could have a chance of getting more papers. (I have done SLR which only produced 3 partially relevant papers. Other methods could have been used, however, my preliminary literature review showed that the literature lacks information about OSRs, therefore, I had to use the web questionnaire instead of choosing another literature review method.)

Page 8: You defined research questions of entire thesis, but there is a need to mention to answer which research questions SLR is being designed. I don't think RQ7 will be included for SLR process. (Appendix A shows a table that maps the research questions to the questions of the questionnaire.)

Page 9: In search string you have stated that you used synonyms of keywords, then why did you not include the term product along with software? Some of the researcher's use the term product development instead of software. Is there any specific reason? If so state it. (I am studying obsolete Software Requirements and not software development or product development. Basically, my topic falls in the requirements phase of software development life cycle.)

Page 9: The problem is that Springer Link does not accept more than 10 search terms meanwhile the search string (mentioned above) contains 12 terms. (No comment was provided)

Page 10: "1) Read the article:" this is step 3 in section 2.1.3, but you named it as step 1. (Corrected)

Page 10: Sections 2.1.1 - 2.1.7: Designing review protocol is a step where you need to report how you plan to conduct systematic literature review, but you reported the results also in this section. Please modify it. In design you need to write how you intend to do SLR, in conducting SLR you need to write how you did SLR and in reports sections you need to report the results of SLR. (No changes, because I did not report my results in this section. Yet, I pointed that the results will be reported in other sections. Which is not a problem, I believe)

Page 11: Section 2.2.1: "The content of the questionnaire was derived from relevant topics of OSRs such as volatility requirements, change management, requirements traceability, and requirements management in general." Which source did you use to get this information? Is it from literature or any industry practitioners? Specify it. If it is from literature, specify did you find relevant papers, as you said you only found three partially relevant papers from SLR and no relevant papers when you conducted a pilot study? (The other topics e.g. volatile requirements are not covered in the SLR. I read several books and papers about requirements engineering and these were my other sources) 
Page 13: You did not specify the objectives for which survey is being conducted. There are types of survey such as exploratory, descriptive, explanatory, and predictive. Specify which type of survey you designed. Which kind of population sampling did you use for selecting participants for answering the survey probability or nonprobability? Specify it with motivation. Are there any outliers for your survey responses? Are there any incomplete responses? What did you do with those responses? (I explicitly mentioned why I used Web Questionnaire, please refer to section 2.2 for more information about it)

Statistical methods:

Page 20: 2.3.Likert Scale (mean and median): What do you mean when you say this? Generally when you refer to something in brackets it means that it is a synonym to the term but in this context Likert scale is a means of measurement, whereas mean and median refers to methods of analysis. There is a chance that reader might get confused looking at this notation. Please try to change it. (Corrected (mean and median) is deleted to eliminate any confusion)

Page 21: Specify type of statistics for chi-square test i.e., it is advanced statistics. What are other available advanced statistics? (This is outside the scope of this master thesis)

Give more motivation explaining how SLR and survey have been connective in your thesis.

More explanation needs to be given as how SLR has benefited your thesis. What is the use of you doing SLR and then the survey rather than directly doing the web survey. Motivate. (This has been motivated in section 2)

\section{Chapter 3: Definition of OSR}

Page 23: definition of OSR: "An obsolete software requirement is a software requirement (implemented or not) that is no longer required for the current release or future releases, and it has no value (business goals) for the potential customers or users of a software product for various reasons." What do you mean by various reasons? Explain it with example. (This text has been added to the definition "e.g. (technological advancements)")

\section{Chapter 4: Requirements classification}

Page 35: What do you mean by company's organization and policies? (the organization of the company can be for instance hierarchical or flat) What about system related requirements? Are they a part of any category? If so, please specify. (system related requirements is a very broad term and it can fall in several classifications e.g. functional and non functional requirements)

\section{Chapter 5: OSR Processes}

Page 45: Actions suggested by the author for OSRs are good. Based on responses it is clear that the some responses are from be-spoke RE and others are from MDRE. How did you generalize the results of your survey to both the contexts? I think it would be better to divide responses based on RE category (be-spoke or MDRE) and check whether the results of respondents are same as that of overall results. I think this then you can generalize the results. (You can generalize such result by using Chi-square test of significance. However, I do not find the way you take action against OSRs is affected by the type of RE you were involved in. Note that other factors such as the role of the respondents were studied in the same chapter.)

\section{Chapter 6: OSR Effects}

Page 52: You said 4 responses have skipped the question, what did you do with the incomplete responses? Do you think these 4 company people do not consider OSR's as an effect due to the size of the project? Give explanation. It makes the reader difficult to understand what you have done with the incomplete responses. (incomplete responses are treated per question. In this case, the researcher 
has to notify the reader how many people did not answer the question. Yet, the analysis is valid since the number is small i.e. only 4 )

Chapter 7: Requirements Engineering process and OSR's

Good explanation as how important OSR is in each phase.

Chapter 8: Conclusion

Page 58: "Another contribution of the thesis is proposing processes or ways of detecting or finding OSRs, as well as suggesting possible actions to be taken against them when they are discovered. Nevertheless, majority of the questionnaire's respondents (54\%) suggest finding OSRs by reading the requirements manually, and after identifying the OSRs mark them as obsolete. Similarly, more than half of the questionnaire's responses agree on keeping OSRs in the documentation, and on providing the rationale why they became obsolete." You have stated that this is in general i.e., applicable to both be-spoke and MDRE, but this is contrast to Regnell's paper Exploring bottlenecks of market-driven requirements engineering, where he states removal of obsolete requirements to handle requirements overload challenge. Can you justify your conclusion in MDRE context? (The conclusion shows the answers of the respondents of my questionnaire. I believe that Regnell makes a mistake by saying removing OSR in order to handle the requirements overload. It is not advised at all to remove any requirements because you need these requirements for future reference. Nevertheless, one can always manage the information in order to reduce the effects of the information overload challenge. Indeed, removing OSRs or any kind of requirement does not solve the problem of information overload.)

\section{Chapter 9: Limitations}

You did not mention limitation for research question 4. Is there no limitation or have you not mentioned it? (It seems that I missed question number. I have added it.)

Page 61: "Additionally, this thesis only studies two dimensions of software projects". What are the other dimensions of the software projects? At least try to mention them. (I added this text in page 62. "Note that other dimensions can be studied in the future such as the development methodology used.")

Chapter 10: Future work

You could have explained how OSR's would help in traceability in a line or two. (This has to be discovered by future work, I would not like to speculate.)

\section{Chapter 11: References}

$4,6,7,16,17,32,33,53,59$, etc: Incomplete references and formatting is also not correct for few. Please check the references format once.

Provide web links for dictionaries mentioned for definitions. (This thesis follows the IEEE citation standard. Corrections were made where applicable.)

\section{Appendix A: mapping research questions with survey questions:}

It seems that you mapped only 9 survey questions with 7 research questions, but you actually had 15 questions in survey. What about the rest 6 questions? If they are not relevant to any RQ what is the purpose of including it in questionnaire? (They are demographic questions and their aim is to study who are the respondents. These questions were used in many cases to study the relationship between for instance the role of the respondent and the existence of software development process that takes OSRs into account.)

\section{General Comments:}


1. Need for breaking lengthy sentences: (The sentences are short, I have used commas in order to shorten them. No changes.)

Examples:

Page 6: According to Kitchenham [23], the goals of SLR are: to summarize existing evidence concerning a research topic, to identify any gaps in the current research areas in order to allow researchers to investigate these gaps and fill them, and to "provide a framework/background in order to appropriately position new research activities"[23].

Page 7: Due to that, the need for SLR (step 1 in Figure 1) emerged in order to collect information and facts about obsolescence of software artefacts, in general, and about obsolescence of software requirements, in particular, in the context of software engineering and requirements engineering, and, to find answers for the research questions (step 2 in Figure 1) of this master thesis (see Table 1).

2. Usage of articles "the" and "a" is not needed that many times. Try to use them when needed. (No considered. Does not make sense.)

3. Page 12 it is ACM not AMC. (Corrected)

4. Page 26 repetition of synonyms already mentioned previously (No changes).

5. Page 46 third paragraph second line they is not needed (No changes).

6. Page 49 sentence framing need to be changed in the first bullet.

7. Page 50 it's not that role its role in CCB line (No changes).

8. Page 50 last but 3rd line what do you mean by statuses? There is no such word. (Statuses is the plural of status)

9. Page 51 9th line this opinion has been confirmed, it should be this opinion has confirmed (Corrected).

Overall Comment:

Your documentation was good. It makes the reader well understand the topic. Good work. 